\title{
Geodesics and metric ball boundaries in Liouville quantum gravity
}

\author{
$\underset{\text { Chicago }}{\underset{\text { Ewain Gwyne }}{\text { Coshua Pfeffer }}} \quad$ Scott $\underset{\text { MIT }}{\text { Sheffield }}$
}

\begin{abstract}
Recent works have shown that there is a canonical way to to assign a metric (distance function) to a Liouville quantum gravity (LQG) surface for any parameter $\gamma \in(0,2)$. We establish a strong confluence property for LQG geodesics, which generalizes a result proven by Angel, Kolesnik and Miermont for the Brownian map. Using this property, we also establish zero-one laws for the Hausdorff dimensions of geodesics, metric ball boundaries, and metric nets w.r.t. the Euclidean or LQG metric. In the case of a metric ball boundary, our result combined with earlier work of Gwynne (2020) gives a formula for the a.s. Hausdorff dimension for the boundary of the metric ball stopped when it hits a fixed point in terms of the Hausdorff dimension of the whole LQG surface. We also show that the Hausdorff dimension of the metric ball boundary is carried by points which are not on the boundary of any complementary connected component of the ball.
\end{abstract}

\section{Contents}

1 Introduction $\quad 2$

1.1 A stronger confluence property for geodesics . . . . . . . . . . . . . . 2

1.2 Zero-one laws for dimensions of geodesics and metric ball boundaries . . . . . . . . 4

1.3 Exterior boundaries of metric balls . . . . . . . . . . . . . . . . . 7

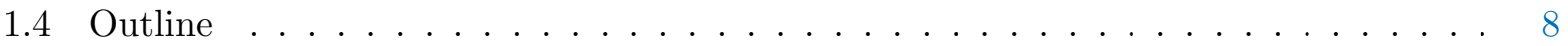

2 Background: definition of the LQG metric $\quad 8$

2.1 Boundaries of filled LQG metric balls are Jordan curves . . . . . . . . . . . . . . 10

3 Strong confluence of LQG geodesics 11

3.1 Confluence at a single point . . . . . . . . . . . . . . . . . 11

3.2 Confluence across metric annuli with finite target points . . . . . . . . . . . 13

3.3 Confluence in a neighborhood of a typical point . . . . . . . . . . . . . 15

4 Zero-one laws for geodesics and ball boundaries: two essential ingredients 20

4.1 Zero-one law for the metric net . . . . . . . . . . . . . . . . . 20

4.2 Scale invariance: defining geodesic rays and metric balls centered at $\infty \ldots \ldots 21$

4.3 Locality: applying the strong confluence property . . . . . . . . . . . . . . . 24

5 Zero-one law for LQG geodesics $\quad 26$

6 LQG metric ball boundaries 30

6.1 A generalized upper bound . . . . . . . . . . . . . . . 30

6.2 The Hausdorff dimension of metric ball boundaries . . . . . . . . . . . . . . . 33

6.3 The exterior boundary of a metric ball . . . . . . . . . . . . . . . 40 


\section{Introduction}

In the 1980s, physicists working in conformal field theory introduced a theory of random surfaces called Liouville quantum gravity (LQG) as canonical models of random two-dimensional Riemannian manifolds [Pol81, Dav88, DK89]. The subject has attracted a substantial amount of mathematical attention in recent years, because of both its relevance to several areas of mathematical physics and its relationship to random discrete surfaces called random planar maps. See [Gwy20c, Ber] for introductory articles on LQG from a mathematical perspective. We can define LQG heuristically as follows.

Definition 1.1 (Heuristic formulation of LQG). Let $\gamma \in(0,2)$. A $\gamma$-Liouville quantum gravity $(\gamma$-LQG) surface is a random Riemannian manifold with random Riemannian metric tensor

$$
e^{\gamma h}\left(d x^{2}+d y^{2}\right)
$$

where $h$ is a variant of the Gaussian free field (GFF) on some domain $U \subset \mathbb{C}$ and $d x^{2}+d y^{2}$ is the Euclidean metric tensor on $U$.

See, e.g., [She07,Ber,WP20] for an introduction to the GFF. The metric tensor (1.1) is not literally well-defined since $h$ is a distribution, not a function, so cannot be exponentiated pointwise. Despite this obstacle, probabilists have rigorously defined both a random measure [Kah85, DS11, RV11] and a random metric (distance function) [DDDF20, GM21b] associated to (1.1) via renormalization procedures. In this paper, we focus on the formulation of LQG as a random metric space, and we describe some fundamental properties of LQG geodesics and metric ball boundaries.

Acknowledgments. We thank an anonymous referee for helpful comments on an earlier version of this paper. E.G. was partially supported by a Clay research fellowship and a Trinity college, Cambridge junior research fellowship. J.P. was partially supported by the National Science Foundation under Grant No. 2002159. S.S. was partially supported by NSF Grant DMS 1712862. No code or data was involved in this work.

\subsection{A stronger confluence property for geodesics}

Throughout this paper, we will focus primarily on the case when $h$ is the whole-plane GFF (results for other variants of the GFF can be extracted via local absolute continuity). The whole-plane GFF is defined only modulo additive constant, but we will almost always fix the constant by requiring that the average of the field over the unit circle is zero.

Miller and Sheffield in [MS20, MS21b, MS21c] defined the metric associated with an LQG surface (i.e., the Riemannian distance function associated with (1.1)) in the special case $\gamma=\sqrt{8 / 3}$. Their work also showed that certain special $\sqrt{8 / 3}$-LQG surfaces are isometric to Brownian surfaces [MS21b, Corollary 1.5]. Brownian surfaces (such as the Brownian map) are random metric spaces that arise as scaling limits of uniform random planar maps with respect to the Gromov-Hausdorff topology; see, e.g., [Le 13, Mie13,BM17]. For several years, this was the only value of $\gamma$ for which mathematicians could define an LQG metric.

More recently, [GM21b] defined the $\gamma$-LQG metric $D_{h}$ for all values of $\gamma$ as the culmination of a long series of papers [DDDF20, GM20b, $\mathrm{DFG}^{+}$20, GM20a, GM21a]. We recall their definition of the $\gamma$-LQG metric in Section 2.

An important difficulty in the study of LQG surfaces is the fact that LQG geodesics are not locally determined by the field $h$, since one needs to see the LQG lengths of all possible paths between two points to see which one has minimal length. One possible way to get around this difficulty is by 
means of confluence of geodesics, which was first established for the LQG metric in [GM20a]. The version of confluence in [GM20a] says that for any fixed point $z$, a.s. any two geodesics started from $z$ (with arbitrary target points) coincide for a non-trivial initial time interval. We note that this is very different from the behavior of geodesics in a smooth Riemannian manifold. Very roughly speaking, confluence of geodesics is used in [GM21b, Section 4] to show that LQG geodesics between typical points are stable in the sense that changing the field $h$ in a small neighborhood of a point on an LQG geodesic is unlikely to result in a macroscopic change to the geodesic. This provides an "approximate Markov property" of LQG geodesics which plays a crucial role in the proof of the uniqueness of the LQG metric. The work [Le 10] proved a similar confluence property for the Brownian map, which was used in the proof that uniform quadrangulations converge to the Brownian map.

Although the above confluence property is useful, it only concerns geodesics started from a fixed point, not geodesics between arbitrary points, so its use is limited when we want to analyze finer properties of the geodesic structure of LQG. In the setting of a Brownian surface, [AKM17] establish several properties of the geodesic structure of the Brownian map by first proving a stronger version of the confluence property [AKM17, Proposition 12]. Roughly speaking, they show that geodesics will merge, not only when started from the same point, but also when started near a typical point. See also [MQ20a] for an even stronger form of confluence for the Brownian map, which holds for geodesics between arbitrary points. Our first result is the analog of the confluence property of [AKM17] for the $\gamma$-LQG metric, for general $\gamma \in(0,2)$ (it remains open to extend the stronger result of [MQ20a] to the case of general $\gamma \in(0,2))$.

Theorem 1.2 (Confluence of LQG geodesics started near a typical point). Let $\gamma \in(0,2)$, let $h$ be the whole-plane GFF and let $D_{h}$ be the associated $\gamma-L Q G$ metric. Almost surely, for each neighborhood $U$ of 0 there is a neighborhood $U^{\prime} \subset U$ of 0 and a point $z_{0} \in U \backslash U^{\prime}$ such that every $D_{h}$-geodesic from a point in $U^{\prime}$ to a point in $\mathbb{C} \backslash U$ passes through $z_{0}$.

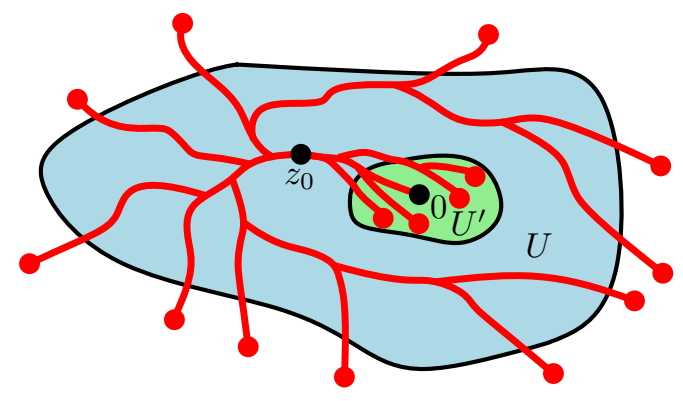

Figure 1: Illustration of the statement of Theorem 1.2. All of the geodesics from points inside the light green region $U^{\prime}$ to points outside the union of the light blue and light green regions pass through the point $z_{0}$ (several such geodesics are shown in red). Note that this is stronger than the confluence result in [GM20a, Theorem 1.3] which only concerns geodesics started from 0 .

See Figure 1 for an illustration of the statement. We prove Theorem 1.2 in Section 3. The proof is in some ways similar to that of the analogous property [AKM17, Proposition 12] of the Brownian map, but with different inputs.

Remark 1.3. Our proof of Theorem 1.2 also works if instead of a whole-plane GFF we consider a whole-plane GFF plus $-\alpha \log |\cdot|$ for $\alpha \in(-\infty, 2 / \gamma+\gamma / 2)$ (this is the range of $\alpha$ values for which the metric is locally finite, see $\left[\mathrm{DFG}^{+} 20\right.$, Theorem 1.10]). In particular, taking $\alpha=\gamma$, we see that our results hold with the origin replaced by a typical point sampled from the LQG area measure. 
This uses the fact that the results of [GM20a] also work for a whole-plane GFF plus $-\alpha \log |\cdot|$, see [GM20a, Remark 1.5].

As in the setting of the Brownian map, the confluence property of Theorem 1.2 is an important ingredient in analyzing the metric properties of LQG. For example, [AKM17] used [AKM17, Proposition 12] to classify the types of geodesic networks that are dense. Specifically, they showed that for each $k \in\{1,2,3,4,6,9\}$, there is a certain topological configuration of exactly $k$ distinct geodesics joining two points which occurs for a dense set of points in the Cartesian product of the Brownian map with itself; and the set of pairs of points joined by any other possible configuration of geodesics is nowhere dense. The paper [Gwy20b] uses Theorem 1.2 to prove the same result for LQG geodesics.

\subsection{Zero-one laws for dimensions of geodesics and metric ball boundaries}

In this paper, we will apply Theorem 1.2 in Sections 4-6, in which we prove results concerning the Hausdorff dimensions of several random fractals associated with the LQG metric. We now describe these results.

Definition 1.4. For a metric space $(X, D)$, a subset $A \subset X$, and a radius $r>0$, we write $\mathcal{B}_{r}(A ; D)$ for the closed metric ball consisting of the set of points in $X$ lying at $D$-distance at most $r$ from $A$. For $A \subset \mathbb{C}$, we write $B_{r}(A)=\mathcal{B}_{r}(A ;|\cdot|)$ for the Euclidean $r$-neighborhood of $z$. When $A=\{x\}$ is a singleton, we abbreviate $\mathcal{B}_{r}(\{x\} ; D)=\mathcal{B}_{r}(x ; D)$ or $B_{r}(\{x\})=B_{r}(x)$.

Definition 1.5 (Definition of Hausdorff dimension). The Hausdorff dimension of a metric space $(X, D)$ is the infimum of the set of $d>0$ such that the following is true: for each $\varepsilon>0$, we can cover $X$ by a collection of balls $\left\{\mathcal{B}_{r_{j}}\left(x_{j} ; D\right)\right\}_{j \in \mathbb{N}}$ for which

$$
\sum_{j \in \mathbb{N}} r_{j}^{d}<\varepsilon
$$

When $X \subset \mathbb{C}$ and $D=|\cdot|$ is the Euclidean metric, we write $\operatorname{dim}_{\mathcal{H}}^{0} X$ for the Hausdorff dimension of $(X,|\cdot|)$ and call it the Euclidean dimension of $X$. When $D=D_{h}$ is the $\gamma$-LQG metric, we write $\operatorname{dim}_{\mathcal{H}}^{\gamma} X$ for the Hausdorff dimension of $\left(X, D_{h}\right)$ and call it the $\gamma$-quantum dimension of $X$.

In the context of LQG, a natural first question to ask is, what is the Hausdorff dimension of the $\gamma$-LQG metric space-i.e., what is the $\gamma$-quantum dimension of $\mathbb{C}$ ? This question has been studied by physicists since the 1990s, long before the rigorous construction of $\gamma$-LQG metric made it possible to state the question rigorously. The value of this dimension as a function of $\gamma$ is not explicitly known except in the case when $\gamma=\sqrt{8 / 3}$, when we know that the dimension is 4 . There is not even a plausible guess for the dimension for other values of $\gamma$ (the best-known physics guess, due to Watabiki [Wat93], was disproven in [DG19]). It is shown in [GP19b] that $\operatorname{dim}_{\mathcal{H}}^{\gamma} \mathbb{C}$ is a.s. equal to the so-called $L Q G$ dimension exponent $d_{\gamma}$ from [DG18, DZZ19], which can be defined in terms of various approximations of LQG (such as random planar maps) and which features in the definition of the $\gamma$-LQG metric. See [DG18, GP19a, Ang19] for the best currently known upper and lower bounds on $d_{\gamma}$ as a function of $\gamma$.

A next natural question is whether there is any relation between the Euclidean and $\gamma$-quantum dimensions of a random fractal set $X \subset \mathbb{C}$. When $X$ is deterministic - or random but independent from the underlying field $h$ - the KPZ formula [KPZ88, DS11, RV11] gives an explicit relationship between $\operatorname{dim}_{\mathcal{H}}^{0} X$ and $\operatorname{dim}_{\mathcal{H}}^{\gamma} X$ in terms of $\gamma$ and $d_{\gamma}$ (see [GP19b] for a proof of the KPZ formula for the metric). However, many of the most natural fractals to study in the LQG setting, such as 
geodesics and metric ball boundaries, are not independent of the underlying field $h$, so the KPZ formula does not apply.

In this paper, we will prove that the Euclidean and $\gamma$-quantum dimensions of several such fractals (such as ball boundaries, geodesics, and the metric net) are a.s. equal to deterministic constants (see theorem statements below). To explain why such zero-one law results are important, we first need to recall the most common approach for computing the Hausdorff dimension of a random fractal, which is based on a result from fractal geometry called Frostman's lemma (see, e.g., [MP10, Theorem $4.32])$.

Lemma 1.6 (Frostman's lemma). A (deterministic) metric space $(X, D)$ has Hausdorff dimension at least $\Delta>0$ if and only if for each $d<\Delta, X$ supports a Frostman measure of dimension d; i.e., a measure $\nu$ on $X$ with positive mass such that

$$
\iint_{X \times X} D(z, w)^{-d} d \nu(z) d \nu(w)<\infty
$$

Probabilists have used Frostman's lemma to compute the a.s. dimensions of many random fractals in Euclidean space, for example the graph of Brownian motion [MP10], Schramm-Loewner evolution curves [Bef08], conformal loop ensembles [MWW16], and thick points of a GFF [HMP10], via the following general approach. To show that the random fractal $X$ in question has Hausdorff dimension $\Delta$ almost surely, one first proves that $\operatorname{dim}_{\mathcal{H}} X \leq \Delta$ by establishing an upper bound for the probability that a given point is "close" to $X$. One then uses first and second moment estimates to prove that, for every $d<\Delta$, the fractal $X$ supports a dimension- $d$ Frostman measure with positive probability. The reason this holds only with positive probability is that the method for proving that the measure has positive mass uses the Payley-Zygmund inequality. Combining this positive probability result and the almost sure upper bound, one gets that $\Delta$ is the essential supremum of $\operatorname{dim}_{\mathcal{H}} X$; i.e.,

$$
\Delta=\sup \left\{d \geq 0: \mathbb{P}\left(\operatorname{dim}_{\mathcal{H}} X \geq d\right)>0\right\} .
$$

To upgrade this to the statement that $\operatorname{dim}_{\mathcal{H}} X=\Delta$ a.s., one typically shows that the dimension of the random fractal $X$ satisfies a zero-one law; i.e., the dimension of $X$ must equal some deterministic constant almost surely. For many interesting random fractals (e.g., many sets defined in terms of SLE or Brownian motion), one has some sort of Markovian or long-range independence property which allows for a relatively straightforward proof of the zero-one law. However, the zero-one laws for many interesting sets associated with the LQG metric are less straightforward, in large part because (as noted above) LQG geodesics are not locally determined by $h$.

The most difficult zero-one law argument in this paper is for the boundary of an LQG metric ball. The essential suprema of the Euclidean and $\gamma$-quantum dimensions of this fractal have already been computed in [Gwy20a]. Combining this with our zero-one law gives the following theorem.

Theorem 1.7 (Dimension of LQG ball boundaries). For each fixed $z \in \mathbb{C}$, a.s. the boundary of the $L Q G$ metric ball $\mathcal{B}_{D_{h}(0, z)}\left(0 ; D_{h}\right)$ centered at 0 and run until it hits $z$ has $\gamma$-quantum dimension

$$
d_{\gamma}-1
$$

and Euclidean dimension

$$
2-\frac{\gamma}{d_{\gamma}}\left(\frac{2}{\gamma}+\frac{\gamma}{2}\right)+\frac{\gamma^{2}}{2 d_{\gamma}^{2}}
$$

(or, equivalently, $2-\xi Q+\xi^{2} / 2$ with $\xi, Q$ defined in (2.2)). 
Since $d \sqrt{8 / 3}=4$, for $\gamma=\sqrt{8 / 3}$ the quantum and Euclidean dimensions in Theorem 1.7 are equal to 3 and $5 / 4$, respectively.

We next consider $\gamma$-LQG geodesics. We know that a.s. the $\gamma$-quantum dimension of every such geodesic equals 1 (the dimension of a geodesic is always equal to 1 w.r.t. the metric for which it is a geodesic). We do not even have a conjecture for the Euclidean dimension, although [GP19b, Corollary 1.10] gives a rigorous upper bound. In this paper we establish the following zero-one law.

Theorem 1.8 (Zero-one law for LQG geodesics). There is a deterministic constant $\Delta_{\text {geo }}>0$ such that a.s. the Euclidean dimension of every $D_{h}$-geodesic started from 0 is equal to $\Delta_{\text {geo }}$.

As a "warm-up" for the proofs of Theorems 1.7 and 1.8, we will also prove a zero-one law for the so-called metric net, which is much easier than the proofs in the case of ball boundaries and geodesics. To define the metric net, we first introduce the notion of a filled metric ball. To motivate the definition, we note that the complement of an LQG metric ball is typically not connected.

Definition 1.9 (The filled metric ball). Let $w \in \mathbb{C}$ and $z \in \mathbb{C} \cup\{\infty\}$. We define the filled metric ball centered at $w$ and targeted at $z$ with radius $s>0$ as

$$
\mathcal{B}_{s}^{z, \bullet}\left(w ; D_{h}\right):= \begin{cases}\text { the union of the closed metric ball } \overline{\mathcal{B}_{s}\left(w ; D_{h}\right)} & \\ \text { and the set of points that this closed } & \text { for } s<D_{h}(w, z) \\ \text { metric ball disconnects from } z & \text { for } s \geq D_{h}(w, z)\end{cases}
$$

We will most often work with filled metric balls centered at zero and filled metric balls targeted at infinity, so to lighten notation, we abbreviate

$$
\mathcal{B}_{s}^{\bullet}\left(w ; D_{h}\right):=\mathcal{B}_{s}^{\infty, \bullet}\left(w ; D_{h}\right), \quad \mathcal{B}_{s}^{z, \bullet}:=\mathcal{B}_{s}^{z, \bullet}\left(0 ; D_{h}\right) \quad \text { and } \quad \mathcal{B}_{s}^{\bullet}:=\mathcal{B}_{s}^{\bullet}\left(0 ; D_{h}\right) .
$$

The metric net is the region of space traced by the boundary of a growing filled metric ball targeted at infinity.

Definition 1.10 (The metric net). The metric net at time $s>0$ is

$$
\mathcal{N}_{s}\left(w ; D_{h}\right):=\bigcup_{t \leq s} \partial \mathcal{B}_{t}^{\bullet}\left(w ; D_{h}\right)
$$

The metric net at time infinity is $\mathcal{N}_{\infty}\left(w ; D_{h}\right):=\bigcup_{s>0} \mathcal{N}_{s}\left(w ; D_{h}\right)$. We abbreviate $\mathcal{N}_{s}:=\mathcal{N}_{s}\left(0 ; D_{h}\right)$.

The metric net at time $s$ is a closed subset of the complex plane. If we were working in a smooth metric space, the metric net would have full Lebesgue measure and Hausdorff dimension; and at time infinity, it would be the entire complex plane. In contrast, the metric net in the LQG metric space has zero Lebesgue measure almost surely, since the probability that any fixed point $z \in \mathbb{C}$ lies on the boundary of the unbounded connected component of $\mathbb{C} \backslash \mathcal{B}_{D_{h}(w, z)}\left(w ; D_{h}\right)$ is zero (see, e.g., the argument of Section 6.3). In Section 4.1, we analyze the $\gamma$-quantum and Euclidean dimension of the metric net, and we show that the scale invariance of the metric net (Lemma 4.1) and a locality property of the metric net (Lemma 4.2) easily imply a zero-one law.

Theorem 1.11. There are deterministic constants $\Delta_{\text {net }}^{0}, \Delta_{\text {net }}^{\gamma}>0$ such that a.s. $\operatorname{dim}_{\mathcal{H}}^{0} \mathcal{N}_{s}=\Delta_{\text {net }}^{0}$ and $\operatorname{dim}_{\mathcal{H}}^{\gamma} \mathcal{N}_{s}=\Delta_{\text {net }}^{\gamma}$ for every $s>0$. 
Proving zero-one laws for LQG geodesics and metric ball boundaries is considerably more challenging: these fractals are neither scale-invariant nor locally determined by the underlying field. We overcome this hurdle as follows.

- Since LQG geodesics and the boundaries of LQG metric balls are not themselves scale-invariant, we instead consider an appropriate "infinite-volume" object whose law is exactly scale invariant. In the case of geodesics, this object is an infinite geodesic ray from 0 to $\infty$. In the case of the metric ball boundary, this object is the boundary of a "metric ball started from $\infty$ and grown until it hits 0", which will be defined as the limit of $\mathcal{B}_{D_{h}(0, w)}\left(w ; D_{h}\right)$ as $w \rightarrow \infty$. We construct these objects in Section 4.2 using the strong confluence of geodesics property established in Theorem 1.2.

- To address the issue that LQG geodesics and the boundaries of LQG metric balls are not locally determined by the underlying field, we again use the strong confluence of geodesics property established in Theorem 1.2. Specifically, we apply this theorem to construct "good" events on which the random fractals are in some sense locally determined (see Lemma 4.8).

This strategy yields zero-one laws (Propositions 4.4 and 6.5) for the infinite-volume versions of LQG geodesics and metric ball boundaries. We then transfer these results from the infinite-volume setting to the finite-volume objects appearing in Theorems 1.7 and 1.8.

For metric ball boundaries, we are able to derive not just a zero-one law, but explicit expressions (1.2) and (1.3) for the Euclidean and $\gamma$-quantum dimensions. This is because we can apply the work of [Gwy20a], which identified (1.2) and (1.3) as the essential suprema of the dimension of an LQG metric ball of a fixed radius with respect to the $\gamma$-LQG and Euclidean metrics, respectively. We apply these results to prove Theorem 1.7 by transferring the results for metric balls of a fixed radius to the case of metric balls run until they hit a fixed point - the type of metric ball for which we have a zero-one law.

Remark 1.12 (Intersection with the the thick points). Following [Gwy20a], for $\alpha \in[-2,2]$ we define the set of metric $\alpha$-thick points of $h$ by

$$
\widehat{\mathcal{T}}_{h}^{\alpha}:=\left\{z \in \mathbb{C}: \lim _{\varepsilon \rightarrow 0} \frac{\log \sup _{u, v \in B_{\varepsilon}(z)} D_{h}(u, v)}{\log \varepsilon}=\frac{\gamma}{d_{\gamma}}(2 / \gamma+\gamma / 2-\alpha)\right\}
$$

where $B_{\varepsilon}(z)$ is the Euclidean ball of radius $\varepsilon$ centered at $z$. The reason for the somewhat strange looking number $\frac{\gamma}{d_{\gamma}}(2 / \gamma+\gamma / 2-\alpha)$ in (1.6) is that this makes it so that $\widehat{\mathcal{T}}_{h}^{\alpha}$ has similar properties to the ordinary $\alpha$-thick points, as considered, e.g., in [HMP10]. Essentially the same arguments as in the proofs of Theorems 1.7, 1.8, and Theorem 1.11 also yield zero-one laws for the Euclidean and $\gamma$-LQG dimensions of the intersection of the random fractals in the theorem statements with $\widehat{\mathcal{T}}_{h}^{\alpha}$. In the setting of Theorem 1.7, we get a formula for the dimension of the intersection in terms of $\gamma, d_{\gamma}, \alpha$ from [Gwy20a, Theorem 1.2]. Similar considerations also apply with the metric thick points replaced by the ordinary thick points (defined using circle averages as in [HMP10, DS11]).

\subsection{Exterior boundaries of metric balls}

Finally, in Section 6.3, we will analyze the exterior boundaries of LQG metric balls.

Definition 1.13 (The exterior boundary of an LQG metric ball). Fix $w \in \mathbb{C}$ and a radius $s>0$, we define the exterior boundary $\mathcal{O}_{s}\left(w ; D_{h}\right)$ of the metric ball $\mathcal{B}_{s}\left(w ; D_{h}\right)$ to be the union of the boundaries of the connected components of $\mathbb{C} \backslash \overline{\mathcal{B}_{s}\left(w ; D_{h}\right)}$. Equivalently, $\mathcal{O}_{s}\left(w ; D_{h}\right)$ is the union of the boundaries of the filled metric balls $\mathcal{B}_{s}^{z, \bullet}\left(w ; D_{h}\right)$ over all $z \in \mathbb{Q}^{2}$. 
For a smooth metric, the notions of boundary and exterior boundary of a metric ball are equivalent. This is not the case for LQG metric balls. The points on the boundary of $\mathcal{B}_{D_{h}(z, w)}\left(w ; D_{h}\right)$ that are not on the exterior boundary arise as accumulation points of connected components of $\mathbb{C} \backslash \mathcal{B}_{s}$ with arbitrarily small diameters. Our main result for exterior boundaries of metric balls is the following theorem, which asserts that "most" points of an LQG metric ball boundary are not in the exterior boundary.

Theorem 1.14. There is a constant $q>0$ such that for each fixed $s>0$ and each fixed $z \in \mathbb{C}$, the Euclidean and $\gamma$-quantum dimensions of $\mathcal{O}_{s}\left(0 ; D_{h}\right)$ are a.s. at most

$$
2-\frac{\gamma}{d_{\gamma}}\left(\frac{2}{\gamma}+\frac{\gamma}{2}\right)+\frac{\gamma^{2}}{2 d_{\gamma}^{2}}-q \text { and } \quad d_{\gamma}-1-q
$$

respectively. The same dimension upper bounds hold for $\mathcal{O}_{D_{h}(0, z)}\left(0 ; D_{h}\right)$. In particular, due to [Gwy20a, Theorem 1.1] (resp. Theorem 1.7), with positive probability (resp. almost surely), the set of points in $\partial \mathcal{B}_{s}\left(0 ; D_{h}\right)$ (resp. $\partial \mathcal{B}_{D_{h}(0, z)}\left(0 ; D_{h}\right)$ ) which do not lie on the boundary of any complementary connected component of $\mathcal{B}_{s}\left(0 ; D_{h}\right)$ (resp. $\mathcal{B}_{D_{h}(0, z)}\left(0 ; D_{h}\right)$ ) has full Hausdorff dimension, hence is uncountable.

The proof of Theorem 1.14, given in Section 6.3, is based on a generalization of the argument used to prove the upper bound for the Euclidean and LQG dimensions of an LQG metric ball boundary in [Gwy20a], see Theorem 6.1.

\subsection{Outline}

In Section 2, we review the definition of the $\gamma$-LQG metric. In Section 3, we prove our main confluence result (Theorem 1.2). In Section 4, we prove a zero-one law for the metric net (Theorem 1.11), and we describe the versions of scale invariance and locality that we will use to prove zero-one laws for LQG geodesics and metric ball boundaries. In Section 5, we prove a zero-one law for geodesics (Theorem 1.8). Finally, in Section 6, we compute the Euclidean and $\gamma$-quantum dimensions of metric ball boundaries (Theorem 1.7), and we study the exterior boundaries of LQG metric balls (Theorem 1.14).

\section{Background: definition of the LQG metric}

In this section, we review the definition of the $\gamma$-LQG metric. The $\gamma$-LQG metric can be defined in two equivalent ways: as the limit of an explicit approximation scheme (called Liouville first passage percolation), and as the unique metric satisfying a list of axioms. In this paper, we will use the axiomatic definition of the LQG metric, which we now state after introducing some metric space terminology.

Definition 2.1 (Terminology for general metric spaces). Let $(X, D)$ be a metric space.

- For a curve $P:[a, b] \rightarrow X$, the $D$-length of $P$ is defined by

$$
\operatorname{len}(P ; D):=\sup _{T} \sum_{i=1}^{\# T} D\left(P\left(t_{i}\right), P\left(t_{i-1}\right)\right)
$$

where the supremum is over all partitions $T: a=t_{0}<\cdots<t_{\# T}=b$ of $[a, b]$. Note that the $D$-length of a curve may be infinite. 
- We say that $(X, D)$ is a length space if for each $x, y \in X$ and each $\varepsilon>0$, there exists a curve of $D$-length at most $D(x, y)+\varepsilon$ from $x$ to $y$.

- For $Y \subset X$, the internal metric of $D$ on $Y$ is defined by

$$
D(x, y ; Y):=\inf _{P \subset Y} \operatorname{len}(P ; D), \quad \forall x, y \in Y
$$

where the infimum is over all paths $P$ in $Y$ from $x$ to $y$. Note that $D(\cdot, \cdot ; Y)$ is a metric on $Y$, except that it is allowed to take infinite values.

- If $X$ is an open subset of $\mathbb{C}$, we say that $D$ is a continuous metric if it induces the Euclidean topology on $X$. We equip the set of continuous metrics on $X$ with the local uniform topology on $X \times X$ and the associated Borel $\sigma$-algebra.

We now define the $\gamma$-LQG metric axiomatically. The definition is phrased in terms of the two parameters $Q$ and $\xi$, defined as

$$
Q=Q_{\gamma}=\frac{2}{\gamma}+\frac{\gamma}{2} \quad \text { and } \quad \xi=\xi_{\gamma}:=\frac{\gamma}{d_{\gamma}}
$$

where, as above, $d_{\gamma}=\operatorname{dim}_{\mathcal{H}}^{\gamma} \mathbb{C}$ is the LQG dimension exponent [DZZ19, DG18, GP19b].

Definition 2.2 (The LQG metric). For $U \subset \mathbb{C}$, let $\mathcal{D}^{\prime}(U)$ be the space of distributions (generalized functions) on $\mathbb{C}$, equipped with the usual weak topology. A $\gamma-L Q G$ metric is a collection of measurable functions $h \mapsto D_{h}$, one for each open set $U \subset \mathbb{C}$, from $\mathcal{D}^{\prime}(U)$ to the space of continuous metrics on $U$ with the following properties. ${ }^{1}$ Let $U \subset \mathbb{C}$ and let $h$ be a $G F F$ plus a continuous function on $U$ : i.e., $h$ is a random distribution on $U$ which can be coupled with a random continuous function $f$ in such a way that $h-f$ has the law of the (zero-boundary or whole-plane, as appropriate) GFF on $U$. Then the associated metric $D_{h}$ satisfies the following axioms.

I. Length space. Almost surely, $\left(U, D_{h}\right)$ is a length space, i.e., the $D_{h}$-distance between any two points of $U$ is the infimum of the $D_{h}$-lengths of $D_{h}$-continuous paths (equivalently, Euclidean continuous paths) in $U$ between the two points.

II. Locality. Let $V \subset U$ be a deterministic open set. The $D_{h}$-internal metric $D_{h}(\cdot, \cdot ; V)$ is a.s. equal to $D_{\left.h\right|_{V}}$, so in particular it is a.s. determined by $\left.h\right|_{V}$.

III. Weyl scaling. Let $\xi=\gamma / d_{\gamma}$ be as in (2.2). For a continuous function $f: U \rightarrow \mathbb{R}$, define

$$
\left(e^{\xi f} \cdot D_{h}\right)(z, w):=\inf _{P: z \rightarrow w} \int_{0}^{\operatorname{len}\left(P ; D_{h}\right)} e^{\xi f(P(t))} d t, \quad \forall z, w \in U,
$$

where the infimum is over all continuous paths from $z$ to $w$ in $U$ parametrized by $D_{h}$-length. Then a.s. $e^{\xi f} \cdot D_{h}=D_{h+f}$ for every continuous function $f: U \rightarrow \mathbb{R}$.

IV. Conformal coordinate change. Let $\widetilde{U} \subset \mathbb{C}$ and let $\phi: U \rightarrow \widetilde{U}$ be a deterministic conformal map. Then, with $Q$ as in (2.2), a.s.

$$
D_{h}(z, w)=D_{h \circ \phi^{-1}+Q \log \left|\left(\phi^{-1}\right)^{\prime}\right|}(\phi(z), \phi(w)), \quad \forall z, w \in U .
$$

\footnotetext{
${ }^{1}$ Our axioms for a $\gamma$-LQG metric only concern a.s. properties of $D_{h}$ when $h$ is a GFF plus a continuous function. So, once we have defined $D_{h}$ a.s. when $h$ is a GFF plus a continuous function, we can take $D$ to be any measurable mapping $\mathcal{D}^{\prime}(U) \rightarrow$ continuous metrics on $U$ \} which is a.s. consistent with our given definition when $h$ is a GFF plus a continuous function. In fact, the construction of the metric in [DDDF20, DFG ${ }^{+} 20$, GM20a, GM21b, GM21a] only gives an explicit definition of $D_{h}$ in the case when $h$ is a GFF plus a continuous function.
} 
The following theorem [DDDF20, GM21b, GM21a] asserts that the $\gamma$-LQG metric defined in Definition 2.2 exists and is unique.

Theorem 2.3 (Existence and uniqueness of the LQG metric). For each $\gamma \in(0,2)$, there exists a metric satisfying the axioms of Definition 2.2. This metric is unique in the following sense: if $D$ and $\widetilde{D}$ are two such metrics, then there is a deterministic constant $C>0$ such that whenever $h$ is a GFF plus a continuous function, a.s. $\widetilde{D}_{h}=C D_{h}$.

More precisely, it is shown in [GM21b, Theorem 1.2], building on [DDDF20, GM20b, DFG ${ }^{+}$20, GM20a], that for each $\gamma \in(0,2)$, there is a measurable function $h \mapsto D_{h}$ from $\mathcal{D}^{\prime}(\mathbb{C})$ to the space of continuous metrics on $\mathbb{C}$ which satisfies the conditions of Definition 2.2 for $U=\mathbb{C}$ (note that this means $\phi$ in Axiom IV is required to be a complex affine map) and is unique in the sense of Theorem 2.3. As explained in [GM21b, Remark 1.5], this gives a way to define $D_{h}$ whenever $h$ is a GFF plus a continuous function on an open domain $U \subset \mathbb{C}$ in such a way that Axioms I through III hold. Note that the metric in the whole-plane case determines the metric on other domains due to Axiom II. It is shown in [GM21a, Theorem 1.1] that with the above definition, Axiom IV holds for general conformal maps.

Because of Theorem 2.3, we may refer to the unique metric satisfying Definition 2.2 as the $\gamma-L Q G$ metric. Technically, the metric is unique only up to a global deterministic multiplicative constant. When referring to the $\gamma$-LQG metric, we are implicitly fixing the constant in some arbitrary way. For example, we could require that the median distance between the left and right sides of $[0,1]^{2}$ is 1 when $h$ is a whole-plane GFF normalized so that its average over the unit circle is zero. The choice of constant will not play any role in our results or proofs.

\subsection{Boundaries of filled LQG metric balls are Jordan curves}

Here we record a basic topological fact about LQG metric balls which will be convenient to use at several places later in the paper. The lemma is a minor variant of [MS21a, Proposition 2.1] and is proven in the same way.

Lemma 2.4. Let $D$ be a length metric on $\mathbb{C}$ which induces the same topology as the Euclidean metric and satisfies $\lim _{z \rightarrow \infty} D(w, z)=\infty$ for some (equivalently, every) $w \in \mathbb{C}$. For $z \in \mathbb{C}$ and $s>0$, write $\mathcal{B}_{s}(z ; D)$ for the $D$-metric ball of radius $s$ centered at $z$. The boundary of each connected component of $\mathbb{C} \backslash \overline{\mathcal{B}_{s}(z ; D)}$ is a Jordan curve.

Proof. Our hypotheses on $D$ imply that $(\mathbb{C}, D)$ is boundedly compact, i.e., each closed $D$-bounded subset of $\mathbb{C}$ is compact. By [BBI01, Corollary 2.5.20], this implies that there is a $D$-geodesic between any two points of $\mathbb{C}$. The lemma now follows from exactly the same argument as [MS21a, Proposition $2.1]$, which is the analogous statement for metrics on the sphere rather than the plane.

Note that the hypotheses of Lemma 2.4 apply a.s. when $D=D_{h}$ is the $\gamma$-LQG metric associated with a whole-plane GFF (see $\left[\mathrm{DFG}^{+} 20\right.$, Lemma 3.8] for a proof that $\lim _{z \rightarrow \infty} D(w, z)=\infty$ ).

We also note that each connected component $U$ of $\mathbb{C} \backslash \overline{\mathcal{B}_{s}(z ; D)}$ is simply connected (since it is connected with connected complement). Since the boundary of such a connected component is a Jordan curve, it follows from Carathéodory's theorem [Pom92, Theorem 2.6] that if $U$ is bounded, then any conformal map $\phi: \mathbb{D} \rightarrow U$ extends to a homeomorphism $\overline{\mathbb{D}} \rightarrow \bar{U}$. If $U$ is unbounded, the same is true with $U$ replaced by $U \cup\{\infty\}$ (viewed as a subset of the Riemann sphere).

Strictly speaking, Lemma 2.4 is not actually necessary in this paper since, whenever we would be inclined to use it, we can work with prime ends instead of actual boundary points. However, the lemma allows us to avoid some technical annoyances and makes some geometric arguments more transparent. 


\section{Strong confluence of LQG geodesics}

The goal of this section is to prove Theorem 1.2. We will make frequent use of the notation for filled LQG metric balls from Definition 1.9.

\subsection{Confluence at a single point}

In this subsection we will review some results concerning confluence of geodesics from [GM20a] and also prove some minor improvements on these results. To do so we first recall the notion of a leftmost geodesic. Each point $x \in \partial \mathcal{B}_{s}^{z, \bullet}\left(w ; D_{h}\right)$ lies at $D_{h}$-distance exactly $s$ from $w$, so every $D_{h}$-geodesic from $w$ to $x$ stays in $\mathcal{B}_{s}^{z, \bullet}\left(w ; D_{h}\right)$. For some points $x$ there might be many such $D_{h}$-geodesics. But, it is shown in [GM20a, Lemma 2.4] that there is always a distinguished $D_{h}$-geodesic from $w$ to $x$, called the leftmost geodesic, which lies (weakly) to the left of every other $D_{h}$-geodesic from $w$ to $x$ if we stand at $x$ and look outward from $\mathcal{B}_{s}^{z, \bullet}\left(w ; D_{h}\right)$. Strictly speaking, [GM20a, Lemma 2.4] only treats the case of filled metric balls targeted at $\infty$, but the same proof works for filled metric balls with different target points.

The following theorem is a compilation of results from [GM20a]. See Figure 2, right, for an illustration.

Theorem 3.1 ( [GM20a]). Almost surely, for every $0<t<s$ the following is true.

1. There is a finite set of points $\mathcal{X}=\mathcal{X}_{t, s} \subset \partial \mathcal{B}_{t}^{\bullet}$ such that every leftmost $D_{h}$-geodesic from 0 to a point of $\partial \mathcal{B}_{s}^{\bullet}$ passes through some $x \in \mathcal{X}$.

2. There is a unique $D_{h}$-geodesic from 0 to $x$ for each $x \in \mathcal{X}$.

3. For $x \in \mathcal{X}$, let $I_{x}$ be the set of $y \in \partial \mathcal{B}_{s}^{\bullet}$ such that that the leftmost $D_{h}$-geodesic from 0 to $y$ passes through $x$. Each $I_{x}$ for $x \in \mathcal{X}$ is a connected arc of $\partial \mathcal{B}_{s}^{\bullet}$ (possibly a singleton) and $\partial \mathcal{B}_{s}^{\bullet}$ is the disjoint union of the arcs $I_{x}$ for $x \in \mathcal{X}$.

4. The counterclockwise cyclic ordering of the arcs $I_{x}$ is the same as the counterclockwise cyclic ordering of the corresponding points $x \in \mathcal{X} \subset \partial \mathcal{B}_{t}^{*}$.

We note that $\partial \mathcal{B}_{s}^{\bullet}$ is a Jordan curve by Lemma 2.4. This allows us to talk about arcs of $\partial \mathcal{B}_{s}^{\bullet}$ without worrying about prime ends, etc.

Proof of Theorem 3.1. Assertion 1 is immediate from [GM20a, Theorem 1.4]. Assertion 2 can be easily deduced from the uniqueness of geodesics between rational points together with the fact that leftmost $D_{h}$-geodesics can be approximated by geodesics to rational points [GM20a, Lemma 2.4]; see the proof of [GM20a, Theorem 3.1]. Assertion 3 follows from [GM20a, Lemma 2.7]. Assertion 4 is implicit in the proof of [GM20a, Lemma 2.7], or alternatively can be extracted from the fact that distinct leftmost $D_{h}$-geodesics from 0 to points of $\partial \mathcal{B}_{s}$ do not cross.

The following minor improvement on Theorem 3.1 allows us to avoid worrying about whether $D_{h}$-geodesics are leftmost and about what happens at the endpoints of the arcs $I_{x}$. Note that now we fix $t$ and $s$, whereas Theorem 3.1 is required to hold simultaneously for all $t$ and $s$.

Proposition 3.2. Fix $0<t<s$ and let $\mathcal{X}=\mathcal{X}_{t, s}$ be the set of confluence points as in Theorem 3.1. Almost surely, for every $D_{h}$-geodesic $P$ from 0 to a point of $\mathbb{C} \backslash \mathcal{B}_{s}^{\bullet}$ there is an $x \in \mathcal{X}$ such that $P(t)=x$ and $P(s)$ is a point of the arc $I_{x}$ which is not one of the endpoints of $I_{x}$. 

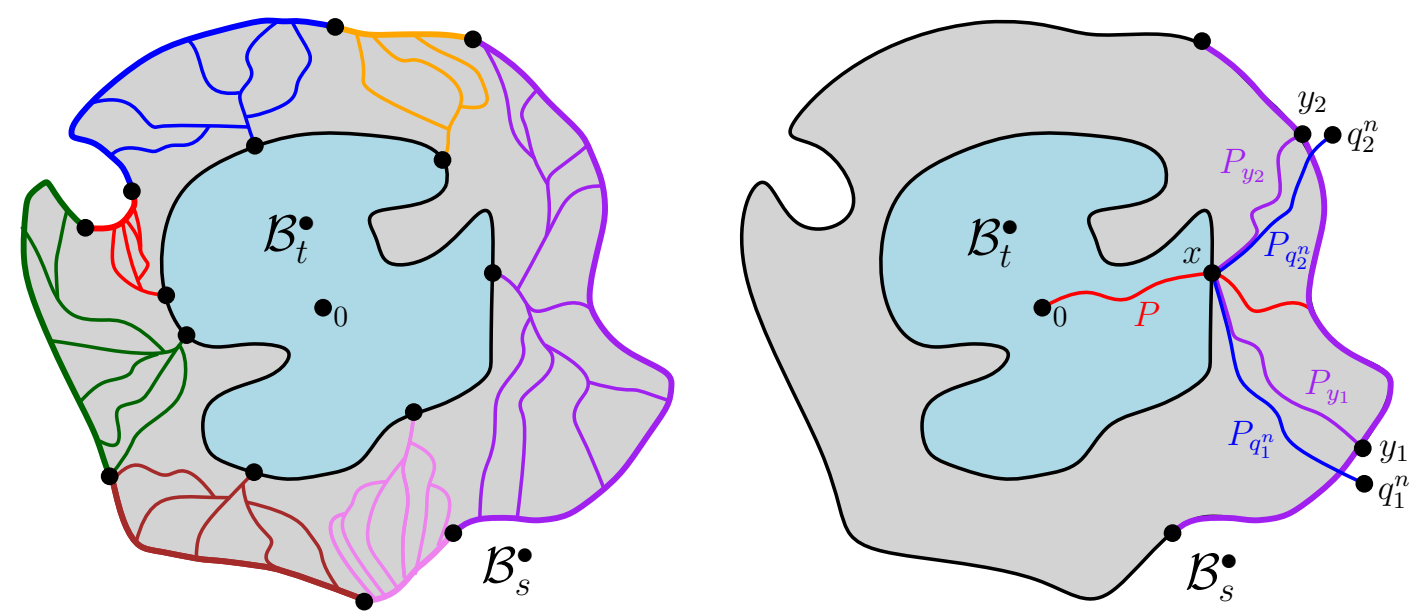

Figure 2: Left: Illustration of the statement of Theorem 3.1. The confluence points $\mathcal{X} \subset \partial \mathcal{B}_{t}^{\bullet}$ are shown in black. For each $x \in \mathcal{X}$, we have shown in a different color the arc $I_{x} \subset \partial \mathcal{B}_{s}^{\bullet}$ and the segments $P([s, t])$ for several representative leftmost $D_{h}$-geodesics $P$ from $x$ to points of $\partial \mathcal{B}_{s}^{\bullet}$. Right: Illustration of the proof of Lemma 3.3. We want to show that the given red $D_{h}$-geodesic $P$ (which is not necessarily a leftmost $D_{h}$-geodesic) passes through $x$. To do this, we construct $D_{h}$-geodesics from 0 to points $q_{1}^{n}, q_{2}^{n} \in \mathbb{Q}^{2} \backslash \mathcal{B}_{s}^{\bullet}$ which pass through $x$ (blue) with the property that if $P$ does not pass through $x$, then it must cross one of these two blue geodesics.

The rest of this subsection is devoted to the proof of Proposition 3.2. We first establish an improvement on Theorem 3.1 which does not require leftmost geodesics.

Lemma 3.3. Almost surely, for each $0<t<s$ the following is true. Define $\mathcal{X}=\mathcal{X}_{t, s}$ and the arcs $I_{x} \subset \partial \mathcal{B}_{s}^{\bullet}$ for $x \in \mathcal{X}$ as in Theorem 3.1. If $P$ is a $D_{h}$-geodesic from 0 to a point of $I_{x}$ (not necessarily a leftmost $D_{h}$-geodesic) and $P(s)$ is not one of the endpoints of $I_{x}$, then $P(t)=x$.

Proof. See Figure 2, right, for an illustration. Throughout the proof, we let $P$ be a geodesic as in the lemma statement and we omit the qualifier "a.s.".

Choose $y_{1}, y_{2} \in I_{x}$ such that the counterclockwise arc of $\partial \mathcal{B}_{s}^{\bullet}$ from $y_{1}$ to $y_{2}$ is contained in $I_{x}$ and contains $P(s)$. We can arrange that neither $y_{1}$ nor $y_{2}$ is equal to $P(s)$ or to one of the endpoints of

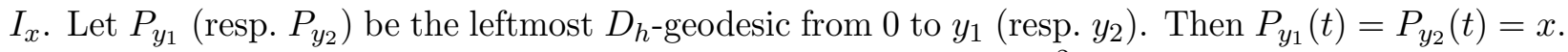
By [GM20a, Lemma 2.4], there are sequences of points $q_{1}^{n}, q_{2}^{n} \in \mathbb{Q}^{2} \backslash \mathcal{B}_{s}^{\bullet}$ such that the following is

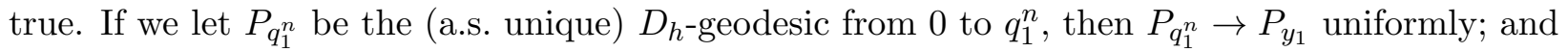
the same is true with 2 in place of 1.

For each $n,\left.P_{q_{1}^{n}}\right|_{[0, s]}$ is the unique (hence leftmost) geodesic from 0 to $q_{1}^{n}$, and the same is true with 2 in place of 1 . Hence each of $P_{q_{1}^{n}}(t)$ and $P_{q_{2}^{n}}(t)$ must belong to the finite set $\mathcal{X}$. By the above uniform convergence for large enough $n$ we have $P_{q_{1}^{n}}(t)=P_{q_{2}^{n}}(t)=x$. Furthermore, for large enough $n$ the counterclockwise arc of $\partial \mathcal{B}_{s}^{\bullet}$ from $P_{q_{1}^{n}}(s)$ to $P_{q_{2}^{n}}(s)$ contains $P(s)$. Henceforth assume $n$ is large enough that these conditions are satisfied.

If $P(t) \neq x$, then topological considerations imply that $P$ must intersect either $P_{q_{1}^{n}}(s)$ or $P_{q_{2}^{n}}(s)$ between time $t$ and time $s$. Assume without loss of generality that $P$ hits $P_{q_{1}^{n}}$. By the uniqueness of $D_{h}$-geodesics to rational points (see [GM20a, Lemma 2.3]) if this is the case then there must be a time $\tau \in[t, s]$ such that $\left.P\right|_{[0, \tau]}=\left.P_{q_{1}^{n}}\right|_{[0, \tau]}$. But, this implies that $P(t)=P_{q_{1}^{n}}(t)=x$.

We now argue that $D_{h}$-geodesics cannot hit the endpoints of the $\operatorname{arcs} I_{x}$. 
Lemma 3.4. Fix $0<t<s$. Almost surely, no $D_{h}$-geodesic from 0 to a point of $\mathbb{C} \backslash \mathcal{B}_{s}^{\bullet}$ hits any of the endpoints of the arcs $I_{x}$ for $x \in \mathcal{X}$.

We will prove Lemma 3.4 using [GM20a, Lemma 3.6], which we re-state just below, and which allows us to prevent $D_{h}$-geodesics from hitting particular points of $\partial \mathcal{B}_{s}^{\bullet}$.

Lemma 3.5 ( [GM20a]). Let $\tau$ be a stopping time for the filtration generated by $\left(\mathcal{B}_{s}^{\bullet},\left.h\right|_{\mathcal{B}_{s}^{\bullet}}\right)$ and let $y \in \partial \mathcal{B}_{\tau}^{\bullet}$ and $\varepsilon \in(0,1)$ be chosen in a $\sigma\left(\mathcal{B}_{\tau}^{\bullet},\left.h\right|_{\mathcal{B}_{\tau}^{\bullet}}\right)$-measurable manner. There exists an event $G_{y}^{\varepsilon} \in \sigma(h)$ and a $\sigma(h)$-measurable random variable $R^{\varepsilon}\left(\mathcal{B}_{\tau}^{\bullet}\right) \in(0, \infty)$ (which does not depend on $y$ ) such that $R^{\varepsilon}\left(\mathcal{B}_{\tau}^{\bullet}\right) \rightarrow 0$ in probability as $\varepsilon \rightarrow 0$ and the following is true.

A. If $R^{\varepsilon}\left(\mathcal{B}_{\tau}^{\bullet}\right) \leq \operatorname{diam} \mathcal{B}_{\tau}^{\bullet}$ and $G_{y}^{\varepsilon}$ occurs, then no $D_{h}$-geodesic from 0 to a point of $\mathbb{C} \backslash B_{R^{\varepsilon}\left(\mathcal{B}_{\tau}^{\bullet}\right)}\left(\mathcal{B}_{\tau}^{\bullet}\right)$ can enter $B_{\varepsilon}(y) \backslash \mathcal{B}_{\tau}^{\bullet}$ (recall that $B_{r}(\cdot)$ denotes a Euclidean neighborhood).

B. There are deterministic constants $C, \alpha>0$ depending only on $\gamma$ such that a.s. $\mathbb{P}\left[G_{y}^{\varepsilon}\left|\mathcal{B}_{\tau}^{\bullet}, h\right|_{\mathcal{B}_{\tau}^{\bullet}}\right] \geq$ $1-C \varepsilon^{\alpha}$.

Proof. This is [GM20a, Lemma 3.6] with $\mathbb{r}=1$. Note that the random variable $R^{\varepsilon}\left(\mathcal{B}_{\tau}^{\bullet}\right)$ is defined in [GM20a, Equation (3.16)] and, as explained just after that equation, it converges to zero in probability as $\varepsilon \rightarrow 0$ by [GM20a, Lemma 3.5].

In the setting of Lemma 3.5, roughly speaking, $G_{y}^{\varepsilon}$ is the event that there is a "shield" around $B_{\varepsilon}(y) \backslash \mathcal{B}_{\tau}^{\bullet}$ in $\mathbb{C} \backslash \mathcal{B}_{\tau}^{\bullet}$ which no $D_{h}$-geodesic started from 0 can pass through. The number $R^{\varepsilon}\left(\mathcal{B}_{\tau}^{\bullet}\right)$ is the maximum possible Euclidean radius of one of these shields.

Proof of Lemma 3.4. Let $\mathcal{Y}$ be the set of endpoints of the arcs $I_{x}$ for $x \in \mathcal{X}$. For $y \in \mathcal{Y}$, let $G_{y}^{\varepsilon}$ be the event of Lemma 3.5 with $\tau=s$. Also let $R^{\varepsilon}\left(\mathcal{B}_{s}^{\bullet}\right)$ be as in Lemma 3.5. Since $\mathcal{Y}$ is a finite, $\sigma\left(\mathcal{B}_{\tau}^{\bullet},\left.h\right|_{\mathcal{B}_{\tau}^{\bullet}}\right)$-measurable set, it follows from Lemma $3.5 \mathrm{~B}$ and the fact that $R^{\varepsilon}\left(\mathcal{B}_{\tau}^{\bullet}\right) \rightarrow 0$ in probability as $\varepsilon \rightarrow 0$ that

$$
\lim _{\varepsilon \rightarrow 0} \mathbb{P}\left[\left\{R^{\varepsilon}\left(\mathcal{B}_{\tau}^{\bullet}\right) \leq \operatorname{diam} \mathcal{B}_{\tau}^{\bullet}\right\} \cap \bigcap_{y \in \mathcal{Y}} G_{y}^{\varepsilon}\right]=1 .
$$

The lemma statement now follows from Lemma 3.5A together with the fact that $R^{\varepsilon}\left(\mathcal{B}_{\tau}^{\bullet}\right) \rightarrow 0$ in probability.

Proof of Proposition 3.2. Let $P$ be a $D_{h}$-geodesic from 0 to a point of $\mathbb{C} \backslash \mathcal{B}_{s}^{\bullet}$. By Lemma 3.4, there is an $x \in \mathcal{X}$ such that $P(s) \in I_{x}$ and $P(s)$ is not one of the endpoints of $I_{x}$. By Lemma 3.3, applied to the geodesic $\left.P\right|_{[0, s]}$, we have $P(t)=x$.

\subsection{Confluence across metric annuli with finite target points}

The results of Section 3.1 concern geodesic across an annulus between two filled metric balls targeted at $\infty$. We now show that the same results are also true for filled metric balls targeted at any $z \in \mathbb{C}$ using the conformal covariance of the metric [GM21a] and local absolute continuity. Due to the translation invariance of the law of $h$, modulo additive constant, it is no loss of generality to restrict attention to filled metric balls centered at 0 . The following is a generalization of Theorem 3.1.

Proposition 3.6. Almost surely, for each $z \in \mathbb{C} \backslash\{0\}$ and each $0<t<s<D_{h}(0, z)$, the following is true.

1. There is a finite set of points $\mathcal{X}=\mathcal{X}_{t, s}^{z} \subset \partial \mathcal{B}_{t}^{z, \bullet}$ such that every leftmost $D_{h}$-geodesic from 0 to a point of $\partial \mathcal{B}_{s}^{z, \bullet}$ passes through some $x \in \mathcal{X}$. 
2. There is a unique $D_{h}$-geodesic from 0 to $x$ for each $x \in \mathcal{X}$.

3. For $x \in \mathcal{X}$, let $I_{x}$ be the set of $y \in \partial \mathcal{B}_{s}^{z, \bullet}$ such that that the leftmost $D_{h}$-geodesic from 0 to $y$ passes through $x$. Each $I_{x}$ for $x \in \mathcal{X}$ is a connected arc of $\partial \mathcal{B}_{s}^{z, \bullet}$ (possibly a singleton) and $\partial \mathcal{B}_{s}^{z, \bullet}$ is the disjoint union of the arcs $I_{x}$ for $x \in \mathcal{X}$.

4. The counterclockwise cyclic ordering of the arcs $I_{x}$ is the same as the counterclockwise cyclic ordering of the corresponding points $x \in \mathcal{X} \subset \partial \mathcal{B}_{t}^{z, \bullet}$.

Proof. The analogous statement for filled metric balls targeted at $\infty$ instead of $z$ is Theorem 3.1. To get the desired statement for filled metric balls targeted at $z$, we use a conformal invariance argument. We first consider a fixed choice of target point $z \in \mathbb{C} \backslash\{0\}$ (we will explain how to get the proposition for all $z$ simultaneously at the end of the proof).

Let $\phi(w):=z w /(w-z)$, so that $\phi(0)=0, \phi(z)=\infty$, and $\phi(\infty)=z$. Define the field

$$
\widetilde{h}:=h \circ \phi^{-1}+Q \log \left|\left(\phi^{-1}\right)^{\prime}\right| .
$$

By the LQG coordinate change formula (Axiom IV), a.s. $D_{\widetilde{h}}(\phi(u), \phi(v))=D_{h}(u, v)$ for each $u, v \in \mathbb{C}$. Consequently, a.s. $D_{\widetilde{h}}(0, z)=\infty$ and

$$
\mathcal{B}_{s}^{z, \bullet}\left(0 ; D_{\widetilde{h}}\right)=\phi\left(\mathcal{B}_{s}^{\bullet}\right), \quad \forall s>0
$$

Therefore, the statement of the proposition is true with $\widetilde{h}$ in place of $h$.

By the conformal invariance of the whole-plane GFF, modulo additive constant, the law of the field $\widetilde{h}$ of (3.2) is that of a whole-plane GFF (with some choice of additive constant) plus a deterministic function which is smooth on $\mathbb{C} \backslash\{0, z\}$. Consequently, for each bounded open set $U \subset \mathbb{C}$ which lies at positive distance from $z$, the laws of $\left.h\right|_{U}$ and $\left.\widetilde{h}\right|_{U}$, viewed modulo additive constant, are mutually absolutely continuous (see, e.g., [MS17, Proposition 2.9]).

Now fix such an open set $U$ with contains 0. By the locality of the metric (Axiom II), if $S>0$ then on the event $\left\{\mathcal{B}_{S} \subset U\right\}$ the metric ball $\mathcal{B}_{S}$ and the restriction $h_{\mathcal{B}_{S}}$ are both a.s. determined by $\left.h\right|_{U}$. Almost surely, each filled metric ball $\mathcal{B}_{s}^{z, \bullet}$ for $s \in[0, S]$ is determined by $\mathcal{B}_{S}$ and $\left.h\right|_{\mathcal{B}_{S}}$. Moreover, each $D_{h}$-geodesic from 0 to a point of $\mathcal{B}_{S}$ is contained in $\mathcal{B}_{S}$, which implies that $D_{h}(0, u)=D_{h}\left(0, u ; \mathcal{B}_{S}\right)$ for each $u \in \mathcal{B}_{S}$.

For $S>0$, let $E_{S}$ be the event that $D_{h}(0, z)<S$ and the four conditions of the proposition statement hold with the given choice of $z$ and for all $0<t<s \leq S$. From the preceding paragraph and Axiom II (locality), we infer that $E_{S}$ is a.s. determined by $\left.h\right|_{U}$ on $\left\{\mathcal{B}_{S} \subset U\right\}$.

By the conclusion of the preceding paragraph and the absolute continuity of the laws of $\left.h\right|_{U}$ and $\left.\widetilde{h}\right|_{U}$, the event $E_{S}$ occurs a.s. on the event $\left\{\mathcal{B}_{S} \subset U\right\}$. Letting $U$ increase to $\mathbb{C} \backslash\{z\}$ and then sending $S \rightarrow \infty$ shows that a.s. the proposition statement holds for our fixed choice of $z$.

We now upgrade to a statement which holds for all $z \in \mathbb{C} \backslash\{0\}$ simultaneously. Indeed, we know from the fixed $z$ case that a.s. the proposition statement holds simultaneously for all $z \in \mathbb{Q}^{2} \backslash\{0\}$. For any $z \in \mathbb{C} \backslash\{0\}$ and any $0<s<D_{h}(0, z)$, there exists $z^{\prime} \in \mathbb{Q}^{2} \backslash \mathcal{B}_{s}^{z, \bullet}$. For such a choice of $z^{\prime}$ we have $\mathcal{B}_{s}^{z, \bullet}=\mathcal{B}_{s}^{z^{\prime}}, \bullet$ for each $t \in[0, s]$. Hence the proposition statement for all $z \in \mathbb{Q}^{2} \backslash\{0\}$ implies the lemma statement for all $z \in \mathbb{C} \backslash\{0\}$.

We will also need an analog of Proposition 3.2 for filled metric balls with arbitrary target points.

Proposition 3.7. Fix $0<t<s$ and let $\mathcal{X}=\mathcal{X}_{t, s}^{z}$ be the set of confluence points as in Proposition 3.6. Almost surely, on the event $\left\{s<D_{h}(0, z)\right\}$, the following is true. For every $D_{h}$-geodesic $P$ from 0 to a point of $\mathbb{C} \backslash \mathcal{B}_{s}^{z, \bullet}$ there is an $x \in \mathcal{X}$ such that $P(t)=x$ and $P(s)$ is a point of the arc $I_{x}$ which is not one of the endpoints of $I_{x}$. 


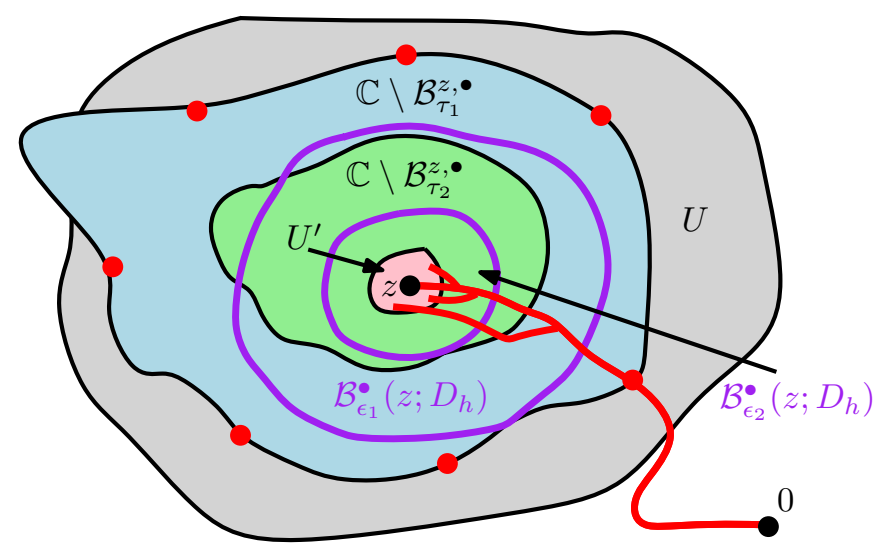

Figure 3: Illustration of the sets involved in the proof of Lemma 3.8. The sets $\mathbb{C} \backslash \mathcal{B}_{\tau_{1}}^{z, \bullet}$ and $\mathbb{C} \backslash \mathcal{B}_{\tau_{2}}^{z, \bullet}$ are shown in blue and green, respectively. The boundaries of the $D_{h}$-balls of radius $\varepsilon_{1}$ and $\varepsilon_{2}$ centered at 0 are shown in purple. The red points are the elements of the set $X$ of points of $\partial \mathcal{B}_{\tau_{1}}^{z, \bullet}$ which are hit by leftmost or rightmost $D_{h}$-geodesics from 0 to $\partial \mathcal{B}_{\tau_{2}}^{z, \bullet}$. This set is finite by Proposition 3.6. The red curves are $D_{h}$-geodesics from 0 to points in $U^{\prime}$, which all coincide outside of $U$.

Proof. For $\varepsilon>0$, let $G(\varepsilon)=G_{t, s}^{z}(\varepsilon)$ be the event that the following is true. For every $D_{h}$-geodesic $P$ from 0 to a point of $\partial \mathcal{B}_{s+\varepsilon}^{z, \bullet}$ there is an $x \in \mathcal{X}$ such that $P(t)=x$ and $P(s)$ is a point of the arc $I_{x}$ which is not one of the endpoints of $I_{x}$. We observe that $G(\varepsilon) \in \sigma\left(\mathcal{B}_{s+\varepsilon}^{z, \bullet},\left.h\right|_{\mathcal{B}_{s+\varepsilon}^{z, \bullet}}\right)$ (due to Axiom II). Furthermore, every $D_{h}$-geodesic from 0 to a point of $\mathbb{C} \backslash \mathcal{B}_{s}^{z, \bullet}$ which lies at $D_{h}$-distance at least $\varepsilon$ from $\partial \mathcal{B}_{s}^{z, \bullet}$ has a sub-path which is a $D_{h}$-geodesic from 0 to a point of $\partial \mathcal{B}_{s+\varepsilon}^{z, \bullet}$. From this, we get that if $\varepsilon^{\prime}<\varepsilon$ then $G\left(\varepsilon^{\prime}\right) \subset G(\varepsilon)$ and moreover the event described in the lemma statement is equal to $\bigcap_{\varepsilon>0} G(\varepsilon)$.

Via exactly the same argument as in the proof of Proposition 3.6, we get that for each fixed $\varepsilon>0$, a.s. $G(\varepsilon)$ occurs on the event $\left\{s+\varepsilon<D_{h}(0, z)\right\}$. Sending $\varepsilon \rightarrow 0$ now concludes the proof.

\subsection{Confluence in a neighborhood of a typical point}

In this subsection we will prove Theorem 1.2, following roughly the argument used to prove the Brownian map analog [AKM17, Proposition 12]. Our next lemma says that any two geodesics started from 0 with nearby target points coincide along a large initial segment. It is the $\gamma$-LQG analog of [AKM17, Lemma 18], but it is proven in a very different manner.

Lemma 3.8. Almost surely, the following is true for each $z \in \mathbb{C}$ such that the $D_{h}$-geodesic from 0 to $z$ is unique. For each open set $U$ containing $z$, there exists an open set $U^{\prime} \subset U$ containing $z$ such that each $D_{h}$-geodesic from 0 to a point of $U^{\prime}$ coincides with the $D_{h}$-geodesic from 0 to $z$ outside of $U$.

We recall that it is shown in [MQ20b, Theorem 1.2] that if $z \in \mathbb{C}$ is fixed, then a.s. the $D_{h}$-geodesic from 0 to $z$ is unique.

Proof of Lemma 3.8. By possibly shrinking $U$, we can assume without loss of generality that $U$ is bounded and that $U$ lies at positive distance from 0 . Let $0<\varepsilon_{2}<\varepsilon_{1}<D_{h}(z, \partial U) / 3$. Let $\tau_{1}$ (resp. $\tau_{2}$ ) be the smallest $s>0$ for which $\mathcal{B}_{s}^{z, \bullet}$ intersects $\mathcal{B}_{\varepsilon_{1}}\left(z ; D_{h}\right)\left(\operatorname{resp} . \mathcal{B}_{\varepsilon_{2}}\left(z ; D_{h}\right)\right)$, so that $0<\tau_{1}<\tau_{2}<D_{h}(0, z)$. See Figure 3 for an illustration. 
By Proposition 3.6 and its analog with rightmost geodesics in place of leftmost geodesics, there is a finite set of points $\mathcal{X} \subset \partial \mathcal{B}_{\tau_{1}}^{z, \bullet}$ such that each leftmost or rightmost $D_{h}$-geodesic from 0 to a point of $\partial \mathcal{B}_{\tau_{2}}^{z, \bullet}$ passes through some $x \in \mathcal{X}{ }^{2}$ Since the $D_{h}$-geodesic $P$ from 0 to $z$ is unique, the

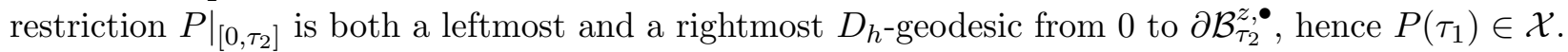

Now consider a sequence of points $z_{n} \rightarrow z$ and for each $n \in \mathbb{N}$ let $P_{n}$ be a $D_{h}$-geodesic from 0 to $z_{n}$ (we do not know that $P_{n}$ is unique). We claim that

$$
P_{n}\left(\tau_{1}\right)=P\left(\tau_{1}\right), \quad \text { for each large enough } n \in \mathbb{N} .
$$

Indeed, each $P_{n}$ is 1-Lipschitz w.r.t. $D_{h}$, so by the Arzéla-Ascoli theorem for every sequence of $n$ 's tending to $\infty$, there is a subsequence along which $P_{n}$ converges uniformly. The uniform limit is necessarily a $D_{h}$-geodesic from 0 to $z$, so must be equal to $P$. Hence $P_{n} \rightarrow P$ uniformly.

Let $P_{n}^{L}$ (resp. $P_{n}^{R}$ ) be the leftmost (resp. rightmost) $D_{h}$-geodesic from 0 to $P_{n}\left(\tau_{2}\right)$. Since $P_{n}\left(\tau_{2}\right) \rightarrow P\left(\tau_{2}\right)$ and the $D_{h}$-geodesic $\left.P\right|_{\left[0, \tau_{2}\right]}$ from 0 to $P\left(\tau_{2}\right)$ is unique, the Arzéla-Ascoli theorem applied as above shows that $P_{n}^{L}$ and $P_{n}^{R}$ each converge uniformly to $\left.P\right|_{\left[0, \tau_{2}\right]}$. Each of the points $P_{n}^{L}\left(\tau_{1}\right), P_{n}^{R}\left(\tau_{1}\right), P\left(\tau_{1}\right)$ belongs to the finite set $\mathcal{X}$, so by the above uniform convergence it follows that for large enough $n \in \mathbb{N}$ we have $P_{n}^{L}\left(\tau_{1}\right)=P_{n}^{R}\left(\tau_{1}\right)=P\left(\tau_{1}\right)$. Since $\left.P_{n}\right|_{\left[0, \tau_{2}\right]}$ lies between $P_{n}^{L}$ and $P_{n}^{R}$, this implies (3.4).

We now deduce from (3.4) that there is an open set $U^{\prime} \subset U$ containing $z$ such that each $D_{h}$-geodesic from 0 to a point of $U^{\prime}$ passes through $P\left(\tau_{1}\right)$. Indeed, if there were no such $U^{\prime}$ then we could find a sequence of $D_{h}$-geodesics from 0 to points arbitrarily close to $z$ which do not pass through $P\left(\tau_{1}\right)$, which would contradict (3.4).

We can assume without loss of generality that the open set $U^{\prime}$ in the preceding paragraph has $D_{h}$-diameter at most $\max \left\{\varepsilon_{1}, D_{h}(z, \partial U)-3 \varepsilon_{1}\right\}$. Then by the definition of $\tau_{1}$ and the triangle inequality, for each $z^{\prime} \in U^{\prime}$,

$$
\begin{aligned}
D_{h}\left(z^{\prime}, \partial U\right) & \geq D_{h}(z, \partial U)-D_{h}\left(z, z^{\prime}\right) \geq 3 \varepsilon_{1} \quad \text { and } \\
D_{h}\left(z^{\prime}, \partial \mathcal{B}_{\tau_{1}}^{z, \bullet}\right) & \leq D_{h}\left(z, \partial \mathcal{B}_{\tau_{1}}^{z, \bullet}\right)+D_{h}\left(z, z^{\prime}\right) \leq 2 \varepsilon_{1} .
\end{aligned}
$$

It follows that no $D_{h}$-geodesic from 0 to $z^{\prime}$ can exit $U$ after time $\tau_{1}$. By the definition of $U^{\prime}$, each such $D_{h}$-geodesic passes through $P\left(\tau_{1}\right)$. By the uniqueness of $P$, it follows that each such $D_{h}$-geodesic in fact coincides with $P$ on $\left[0, \tau_{1}\right]$. Therefore, each such $D_{h}$-geodesic coincides with $P$ outside of $U$, as required.

The following lemma will allow us to apply Proposition 3.6 to sub-segments of general geodesics started from 0 .

Lemma 3.9. Almost surely, the following is true for each $z \in \mathbb{C}$ and each $D_{h}$-geodesic $P$ : $\left[0, D_{h}(0, z)\right] \rightarrow \mathbb{C}$ from 0 to $z$. For each $0<r<D_{h}(0, z)$, the segment $\left.P\right|_{[0, r]}$ is the only $D_{h}$-geodesic from 0 to $P(r)$.

Proof. Let $q \in \mathbb{Q}^{2}$ and radii $0<t<s$ with $t, s \in \mathbb{Q}$. We claim that on the event $\left\{s<D_{h}(0, q)\right\}$, a.s. the following is true. For each $z \in \mathbb{C} \backslash \mathcal{B}_{s}^{q, \bullet}$ and each $D_{h}$-geodesic $P$ from 0 to $z,\left.P\right|_{[0, t]}$ is the only $D_{h}$-geodesic from 0 to $P(t)$. To see this, let $\mathcal{X}=\mathcal{X}_{t, s}^{q} \subset \partial \mathcal{B}_{t}^{\bullet}$ be the set of confluence points as in Proposition 3.6. By assertion 2 of Proposition 3.6, for each $x \in \mathcal{X}$ there is a unique $D_{h}$-geodesic from 0 to $x$. By Proposition 3.7, a.s. each $D_{h}$-geodesic $P$ from 0 to a point of $\mathbb{C} \backslash \mathcal{B}_{s}^{q, \bullet}$ passes through some $x \in \mathcal{X}$. Hence $\left.P\right|_{[0, t]}$ must be the unique $D_{h}$-geodesic from 0 to $P(t)=x$, as required.

\footnotetext{
${ }^{2}$ We cannot apply Proposition 3.7 instead of Proposition 3.6 here since the radii $\tau_{1}$ and $\tau_{2}$ are random.
} 

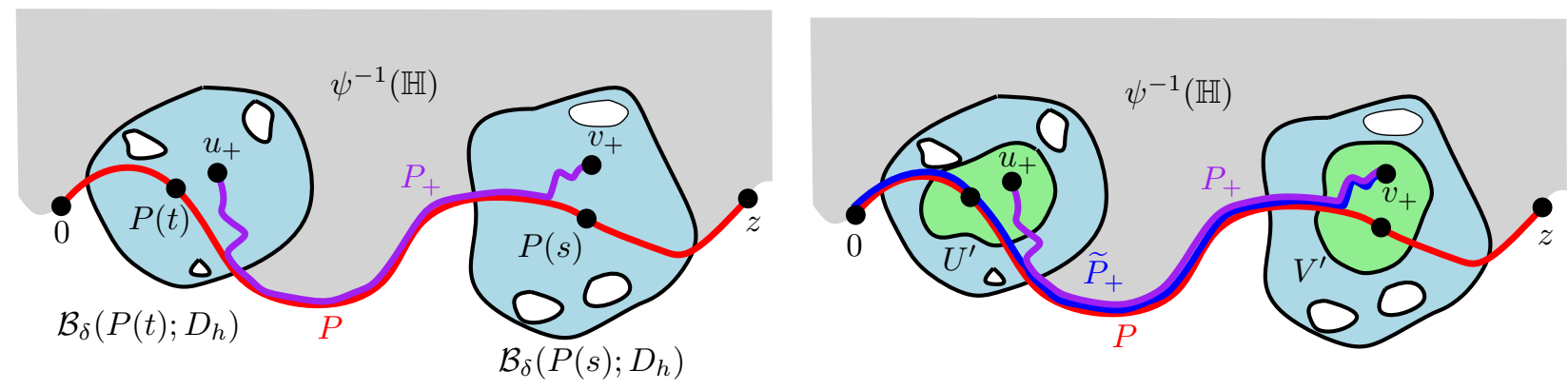

Figure 4: Left. Illustration of the statement of Lemma 3.10. The lemma asserts the existence of the purple $D_{h}$-geodesic in the figure. Right. Illustration of the proof of Lemma 3.10. We first apply Lemma 3.8 with $P(s)$ in place of $z$ and $\mathcal{B}_{\delta}\left(P(s) ; D_{h}\right)$ in place of $U$ to produce the open set $V^{\prime}$. We then apply Lemma 3.8 a second time with $v_{+} \in V^{\prime}$ in place of zero, $P(t)$ in place of $z$, and $\mathcal{B}_{\delta}\left(P(t) ; D_{h}\right)$ in place of $U$ to produce the open set $U^{\prime}$. Note that the red, blue, and purple $D_{h}$-geodesics all coincide outside of the union of the light blue $D_{h}$-metric balls.

Let us now deduce the lemma statement from the above claim. The event described in the above claim holds a.s. for every $q \in \mathbb{Q}^{2}$ and every $0<t<s<D_{h}(0, q)$ with $t, s \in \mathbb{Q}$. We work on the (full probability) event that this is the case. For $z \in \mathbb{C}$ and $0<r<D_{h}(0, z)$, we choose $t, s \in \mathbb{Q}$ such that $r<t<s<D_{h}(0, z)$. The point $z$ lies in a connected component of $\mathbb{C} \backslash \mathcal{B}_{s}$, which is necessarily equal to $\mathbb{C} \backslash \mathcal{B}_{s}^{q, \bullet}$ for some $q \in \mathbb{Q}^{2}$. By the above claim applied to this choice of $q, t, s$, we get that $\left.P\right|_{[0, t]}$ is the only $D_{h}$-geodesic from 0 to $P(t)$. Since $r<t$, this implies that $\left.P\right|_{[0, r]}$ is the only $D_{h}$-geodesic from 0 to $P(r)$ (otherwise, we could obtain two distinct geodesics from 0 to $P(t)$ by concatenating geodesics from 0 to $P(r)$ with $\left.\left.P\right|_{[r, t]}\right)$.

The rest of the proof of Theorem 1.2 is very similar to the proof of [AKM17, Proposition 12]. We will work with the following setup. Fix $z \in \mathbb{C} \backslash\{0\}$ and a $D_{h}$-geodesic $P$ from 0 to $z$. All of the almost sure statements below are required to hold for every choice of $z$ and $P$ simultaneously. To simplify the geometry of the problem, we also fix a homeomorphism $\psi: \mathbb{C} \rightarrow \mathbb{C}$ which takes the geodesic $P$ to the line segment $[0,1]$. The existence of such a homeomorphism follows from general topological theorems, as explained in [AKM17, Section 3]. The following lemma asserts the existence of an "auxiliary" geodesic which will allow us to force $D_{h}$-geodesics near $P$ to merge into $P$. See Figure 4, left, for an illustration of the statement.

Lemma 3.10. Let $0<t<s<D_{h}(u, v)$. Almost surely, for each $\delta>0$ there are points $u_{+} \in$ $\mathbb{Q}^{2} \cap \mathcal{B}_{\delta}\left(P(t) ; D_{h}\right)$ and $v_{+} \in \mathbb{Q}^{2} \cap \mathcal{B}_{\delta}\left(P(s) ; D_{h}\right)$ such that if $P_{+}$is the (a.s. unique) $D_{h}$-geodesic from $u_{+}$to $v_{+}$, then

$$
P_{+} \backslash P \subset\left[\mathcal{B}_{\delta}\left(P(t) ; D_{h}\right) \cup \mathcal{B}_{\delta}\left(P(s) ; D_{h}\right)\right] \cap \psi^{-1}(\mathbb{H})
$$

Proof. We can assume without loss of generality that $\delta<(s-t) / 100$, so that $P(s) \notin \mathcal{B}_{\delta}\left(P(t) ; D_{h}\right)$ and the same is true with $s$ and $t$ interchanged.

By Lemma 3.9, $\left.P\right|_{[0, s]}$ is a.s. the unique $D_{h}$-geodesic from 0 to $P(s)$. Hence Lemma 3.8 (applied with $P(s)$ in place of $z)$ implies that there is an open set $V^{\prime} \subset \mathcal{B}_{\delta}\left(P(s) ; D_{h}\right)$ containing $P(s)$ such that each $D_{h}$-geodesic from 0 to a point of $V^{\prime}$ coincides with $\left.P\right|_{[0, s]}$ outside of $\mathcal{B}_{\delta}\left(P(s) ; D_{h}\right)$. Let $v_{+} \in V^{\prime} \cap \mathbb{Q}^{2} \cap \psi^{-1}(\mathbb{H})$ and let $\widetilde{P}_{+}$be the $D_{h}$-geodesic from 0 to $v_{+}$(which is a.s. unique, since $\left.v_{+} \in \mathbb{Q}^{2}\right)$. Note that the symmetric difference of $\widetilde{P}_{+}$and $P([0, s])$ is contained in $\mathcal{B}_{\delta}\left(P(s) ; D_{h}\right)$. 


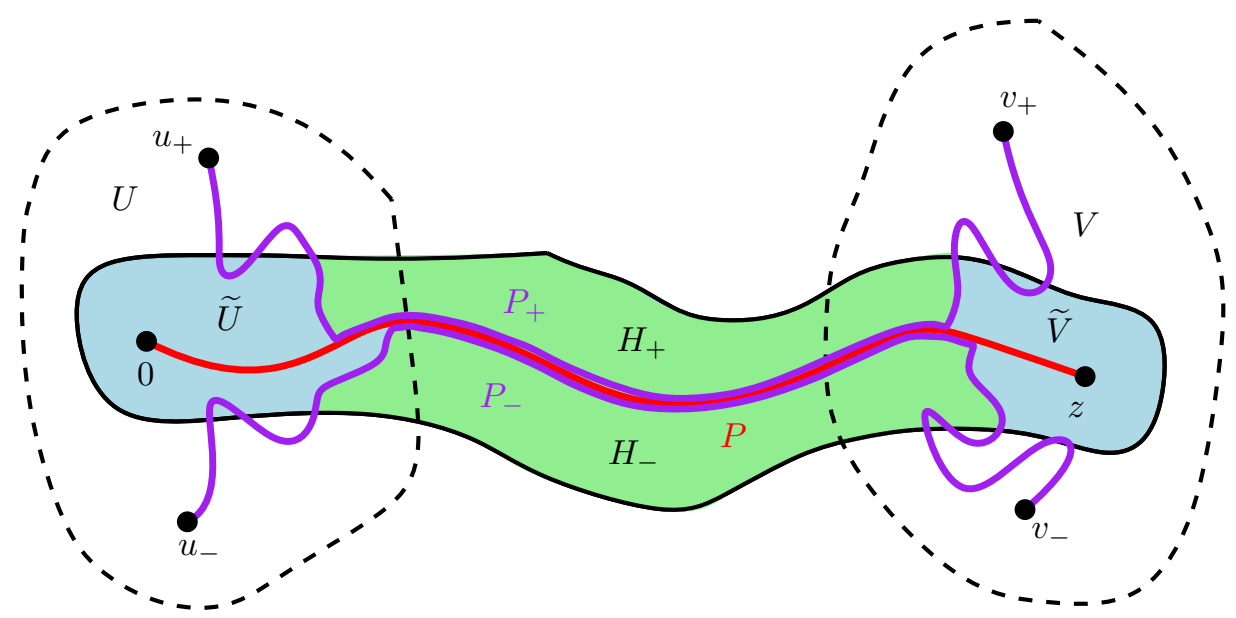

Figure 5: Illustration of the proof of Lemma 3.11. The purple $D_{h}$-geodesics $P_{+}$and $P_{-}$come from Lemma 3.10. They divide the region $\psi^{-1}\left(B_{\varepsilon}([0,1])\right)$ (light green and light blue, not labelled) into four sub-regions $\widetilde{U}, \widetilde{V}, H_{+}, H_{-}$. For large enough $n$, the $D_{h}$-geodesic $P_{n}$ must be contained in $\psi^{-1}\left(B_{\varepsilon}([0,1])\right)$ and its initial (resp. terminal) point must be contained in $\widetilde{U}$ (resp. $\widetilde{V}$ ). Furthermore, due to the uniqueness of the $D_{h}$-geodesics between $u_{+}$and $v_{+}$and between $u_{-}$and $v_{-}$this $D_{h}$-geodesic cannot enter $H_{+} \cup H_{-}$. This forces $P_{n}$ to merge into $P$.

Since $P(t) \notin \mathcal{B}_{\delta}\left(P(s) ; D_{h}\right)$, we have $P(t)=\widetilde{P}_{+}(t)$. Almost surely, the $D_{h}$-geodesic from $v_{+}$to $P(t)$ is unique (it coincides with a segment of the time reversal of $\widetilde{P}_{+}$) since otherwise there would be more than one $D_{h}$-geodesic from 0 to $v_{+}$. Since $v_{+} \in \mathbb{Q}^{2}$, we can apply Lemma 3.8 with $v_{+}$in place of 0 and $P(t)$ in place of $z$ to find that a.s. there is an open set $U^{\prime} \subset \mathcal{B}_{\delta}\left(P(t) ; D_{h}\right)$ containing $P(t)$ such that each $D_{h}$-geodesic from $v_{+}$to a point of $U^{\prime}$ coincides with a segment of $\widetilde{P}_{+}$outside of $\mathcal{B}_{\delta}\left(P(t) ; D_{h}\right)$. We now choose $u_{+} \in U^{\prime} \cap \mathbb{Q}^{2} \cap \psi^{-1}(\mathbb{H})$ and let $P_{+}$be the (a.s. unique, since

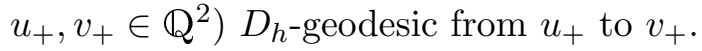

By our choice of $U^{\prime}$, the geodesic $P_{+}$coincides with a segment of $P_{+}$outside of $\mathcal{B}_{\delta}\left(P(t) ; D_{h}\right)$, which in turn coincides with a segment of $\left.P\right|_{[0, s]}$ outside of $\mathcal{B}_{\delta}\left(P(s) ; D_{h}\right)$. Therefore,

$$
P_{+} \backslash P \subset \mathcal{B}_{\delta}\left(P(t) ; D_{h}\right) \cup \mathcal{B}_{\delta}\left(P(s) ; D_{h}\right) .
$$

It remains to show that $P_{+} \backslash P \subset \psi^{-1}(\mathbb{H})$. By possibly shrinking $\delta$, we can assume without loss of generality that $0, z \notin \mathcal{B}_{\delta}\left(P(t) ; D_{h}\right) \cup \mathcal{B}_{\delta}\left(P(s) ; D_{h}\right)$ and $\mathcal{B}_{\delta}\left(P(t) ; D_{h}\right) \cap \mathcal{B}_{\delta}\left(P(s) ; D_{h}\right)=\emptyset$. Since $\mathcal{B}_{\delta}\left(P(t) ; D_{h}\right)$ and $\mathcal{B}_{\delta}\left(P(s) ; D_{h}\right)$ are connected, this implies that no path in $\psi\left(\mathcal{B}_{\delta}\left(P(t) ; D_{h}\right)\right)$ or $\psi\left(\mathcal{B}_{\delta}\left(P(s) ; D_{h}\right)\right)$ can cross $\psi^{-1}(\mathbb{R})$ without first crossing $P$. The set of times $t$ for which $P_{+}(t) \in P$ must be an interval, for otherwise by replacing a segment of $P_{+}$by a segment of $P$ we would violate the uniqueness of $P$. If follows that the segments of $P_{+}$contained in $\mathcal{B}_{\delta}\left(P(t) ; D_{h}\right)$ and $\mathcal{B}_{\delta}\left(P(s) ; D_{h}\right)$ cannot cross $P$. From (3.6) and since $\psi\left(u_{+}\right), \psi\left(v_{+}\right) \in \mathbb{H}$, it therefore follows that $\psi\left(P_{+} \backslash P\right) \subset \mathbb{H}$, as required.

Lemma 3.11. Almost surely, the following is true simultaneously for each $z \in \mathbb{C}$, each neighborhood $U$ of 0 , and each neighborhood $V$ of $z$. Let $P$ be a $D_{h}$-geodesic from 0 to $z$ and let $\left\{P_{n}\right\}_{n \in \mathbb{N}}$ be a sequence of $D_{h}$-geodesics which converge uniformly to $P$. Then

$$
P_{n} \backslash(U \cup V) \subset P, \quad \text { for each large enough } n \in \mathbb{N} .
$$

Proof. Choose $0<s<t<D_{h}(z, w)$ and $\delta>0$ so that $\mathcal{B}_{\delta}\left(P(t) ; D_{h}\right) \subset U, \mathcal{B}_{\delta}\left(P(s) ; D_{h}\right) \subset V$, and $\mathcal{B}_{\delta}\left(P(t) ; D_{h}\right)$ and $\mathcal{B}_{\delta}\left(P(s) ; D_{h}\right)$ lie at positive distance from each other and from 0 and $z$. Define 
$u_{+}, v_{+}$, and $P_{+}$as in Lemma 3.10 and let $u_{-}, v_{-}$, and $P_{-}$be as in Lemma 3.10 with $-\mathbb{H}$ in place of $\mathbb{H}$, so that $P_{-}$is the unique $D_{h}$-geodesic from $u_{-}$to $v_{-}$and $P_{-} \backslash P \subset\left[\mathcal{B}_{\delta}\left(P(t) ; D_{h}\right) \cup \mathcal{B}_{\delta}\left(P(s) ; D_{h}\right)\right] \cap$ $\psi^{-1}(-\mathbb{H})$.

Recall that $\psi(P)=[0,1]$, so we can find $\varepsilon>0$ small enough that $u_{+}, v_{+}, u_{-}, v_{-} \notin \psi^{-1}\left(B_{\varepsilon}([0,1])\right)$. Let $P_{ \pm}^{\prime}$ be the segment of $P_{ \pm}$between the last time that it enters $\psi^{-1}\left(B_{\varepsilon}([0,1])\right)$ before hitting $P$ and the first time that it exits $\psi^{-1}\left(B_{\varepsilon}([0,1])\right)$ after hitting $P$. The set $\psi^{-1}\left(B_{\varepsilon}([0,1])\right)$ is homeomorphic to the open disk and $P_{ \pm}^{\prime}$ is a simple curve joining two of its boundary points, so $P_{ \pm}^{\prime}$ divides $\psi^{-1}\left(B_{\varepsilon}([0,1])\right)$ into two connected components. Let $H_{ \pm}$be the one of these two connected components which is contained in $\psi^{-1}( \pm \mathbb{H})$.

Since $\mathcal{B}_{\delta}\left(P(t) ; D_{h}\right)$ and $\mathcal{B}_{\delta}\left(P(s) ; D_{h}\right)$ lie at positive distance from each other, it follows from the defining property of $P_{ \pm}$that $\partial H_{+} \cap \partial H_{-}$is a non-trivial segment of $P$. Furthermore, since $\mathcal{B}_{\delta}\left(P(t) ; D_{h}\right)$ and $\mathcal{B}_{\delta}\left(P(s) ; D_{h}\right)$ lie at positive distance from 0 and $z$, it is easily seen that 0 and $z$ each lie at positive distance from $P_{+} \cup P_{-}$and hence also from $\bar{H}_{+} \cup \bar{H}_{-}$. From this, we see that $\psi^{-1}\left(B_{\varepsilon}([0,1])\right) \backslash\left(\bar{H}_{+} \cup \bar{H}_{-}\right)$is the union of two open sets $\widetilde{U}, \widetilde{V}$ which lie at positive distance from each other such that $0 \in \widetilde{U}$ and $z \in \widetilde{V}$. Since $P_{+} \backslash P$ and $P_{-} \backslash P$ are each contained in $U \cup V$, we have $\widetilde{U} \subset U$ and $\widetilde{V} \subset V$.

Now consider a sequence of $D_{h}$-geodesics $P_{n} \rightarrow P$ as in the lemma statement. For each large enough $n \in \mathbb{N}, P_{n}$ is entirely contained in $\psi^{-1}\left(B_{\varepsilon}([0,1])\right)$ and the starting and ending points of $P_{n}$ are contained in $\widetilde{U}$ and $\widetilde{V}$, respectively. Henceforth assume that this is the case. Then $P_{n}$ must pass through $\bar{H}_{+} \cup \bar{H}_{-}$.

For any two times $\tau<\sigma$ for which $P_{n}(\tau), P_{n}(\sigma) \in P_{+}$, it must be the case that $P_{n}$ traces $P_{+}$during the time interval $[\tau, \sigma]$ : indeed, otherwise there would be two distinct $D_{h}$-geodesics between the endpoints of $P_{+}$. If $P_{n}$ enters $H_{+}$, then it must subsequently exit $H_{+}$and hence it must hit $\partial H_{+} \cap \psi^{-1}\left(B_{\varepsilon}([0,1])\right) \subset P_{+}$twice. Hence $P_{n}$ cannot enter $H_{+}$. Similarly, $P_{n}$ cannot enter $H_{-}$. Since $P_{n} \subset \psi^{-1}\left(B_{\varepsilon}([0,1])\right)$, we therefore get $P_{n} \subset \widetilde{U} \cup \widetilde{V} \cup P_{+} \cup P_{-}$which implies that $P_{n} \backslash(U \cup V) \subset\left(P_{+} \cup P_{-}\right) \backslash(U \cup V) \subset P$. Thus (3.7) holds.

Lemma 3.12. Almost surely, for each $z \in \mathbb{C}$, each neighborhood $U$ of 0 , and each neighborhood $V$ of $z$, there are open sets $U^{\prime}, V^{\prime}$ with $0 \in U^{\prime} \subset U$ and $z \in V^{\prime} \subset V$ such that every $D_{h}$-geodesic from a point of $U^{\prime}$ to a point of $V^{\prime}$ coincides with a $D_{h}$-geodesic from 0 to $z$ outside of $U \cup V$.

Proof. Assume by way of contradiction that no sets $U^{\prime}, V^{\prime}$ in the lemma statement exist. Then there is a sequence of points $w_{n} \rightarrow 0$, a sequence of points $z_{n} \rightarrow z$, and a sequence of $D_{h}$-geodesics $P_{n}$ from 0 to $z$ such that $P_{n} \backslash(U \cup V)$ is not contained in $P$ for any $n \in \mathbb{N}$. By the Arzéla-Ascoli theorem, after possibly passing to a subsequence, we can arrange that $P_{n}$ converges uniformly to a path $P$ from 0 to $z$ which is necessarily a $D_{h}$-geodesic from 0 to $z$. We then obtain a contradiction from Lemma 3.11.

Proof of Theorem 1.2. By possibly shrinking $U$, we can assume without loss of generality that $U$ is bounded. By [GM20a, Theorem 1.3], a.s. there exists $z_{0} \in U \backslash\{0\}$ such that every $D_{h}$-geodesic from 0 to a point of $\mathbb{C} \backslash U$ passes through $z_{0}$. By Lemma 3.12 (applied with each of $U$ and $V$ replaced by $\mathbb{C} \backslash\left\{z_{0}\right\}$ ), for each $z \in \partial U$ we can choose a neighborhood $U_{z}^{\prime}$ of 0 and a neighborhood $V_{z}^{\prime}$ of $z$ such that $z_{0} \notin U_{z}^{\prime} \cup V_{z}^{\prime}$ and each $D_{h}$-geodesic from a point of $U_{z}^{\prime}$ to a point of $V_{z}^{\prime}$ coincides with a $D_{h}$-geodesic from 0 to $z$ outside of $U_{z}^{\prime} \cup V_{z}^{\prime}$. In particular, each such $D_{h}$-geodesic must pass through $z_{0}$.

By the compactness of $\partial U$, there is a finite set $Z$ of points in $\partial U$ such that $\partial U \subset \bigcup_{z \in Z} V_{z}^{\prime}$. Hence, if we set $U^{\prime}=\bigcap_{z \in Z} U_{z}^{\prime}$, then every $D_{h}$-geodesic from a point in $U^{\prime}$ to a point in $\partial U$ must pass through $z_{0}$. Every $D_{h}$-geodesic from a point in $U^{\prime}$ to a point in $\mathbb{C} \backslash U$ has a sub-segment which 
is a $D_{h}$-geodesic from 0 to a point of $\partial U^{\prime}$. Hence, the theorem statement holds for this choice of $U^{\prime}$.

\section{Zero-one laws for geodesics and ball boundaries: two essential ingredients}

As we described in Section 1.2, the two ingredients we need to prove zero-one laws for random fractals are (1) a version of scale invariance, and (2) a locality property. We demonstrate the basic idea of our proofs in Section 4.1 by proving Theorem 1.11, the zero-one law for the metric net.

In the context of the LQG metric space, we are most interested in LQG geodesics and LQG ball boundaries; but these fractals are neither scale-invariant nor local. We address these two issues in Sections 4.2 and 4.3 in the manner discussed just after the statement of Theorem 1.11. We then prove our results for LQG geodesics (Theorem 1.8) and LQG ball boundaries (Theorem 1.7) in Sections 5 and 6 , respectively.

\subsection{Zero-one law for the metric net}

As we mentioned in Section 1.2, the metric net satisfies both a scale invariance property and a locality property.

Lemma 4.1 (Scale invariance of the metric net). For each $r>0$, a.s.

$$
r \mathcal{N}_{r^{-\xi Q_{e}} e^{-\xi h_{r}(0)} s}\left(0 ; D_{h(r \cdot)-h_{r}(0)}\right)=\mathcal{N}_{s}, \quad \forall s>0 .
$$

In particular, $\left(r \mathcal{N}_{s}\right)_{s \geq 0}$ has the same law as $\left(\mathcal{N}_{s}\right)_{s \geq 0}$ modulo a linear change of the time parametrization.

Proof. By Axioms III and IV (Weyl scaling and coordinate change), a.s.

$$
D_{h(r .)-h_{r}(0)}(u, v)=r^{-\xi Q} e^{-\xi h_{r}(0)} D_{h(r \cdot)+Q \log r}(u, v)=r^{-\xi Q} e^{-\xi h_{r}(0)} D_{h}(r u, r v), \quad \forall u, v \in \mathbb{C} .
$$

From this the relation (4.1) is immediate. Since $h(r \cdot)-h_{r}(0) \stackrel{d}{=} h$, the last statement follows from (4.1).

Lemma 4.2 (Locality of the metric net). For $r>0$, let

$$
\tau_{r}:=\inf \left\{s>0: \mathcal{B}_{s} \not \subset B_{r}(0)\right\}=\inf \left\{s>0: \mathcal{N}_{s} \not \subset B_{r}(0)\right\} .
$$

For each $r>0, \mathcal{N}_{\tau_{r}}$ is a.s. determined by $\left.h\right|_{B_{\tau_{r}}(0)}$

Proof. This is immediate from the locality property of the LQG metric (Axiom II).

Theorem 1.11 follows easily from these two properties of the metric net. Roughly speaking, we derive the zero-one law as follows.

- The scale invariance property of $\mathcal{N}_{s}$ (Lemma 4.1) allows us to get lower bounds for the probability that the Hausdorff dimension is bounded below which hold uniformly over all Euclidean scales.

- The locality property (Lemma 4.2) allows us to use the tail triviality of the $\sigma$-algebras $\sigma\left(\left.h\right|_{B_{r}(0)}\right)$ as $r \rightarrow 0$ to upgrade from positive probability to probability one. 
Proof of Theorem 1.11. We prove the result for Euclidean dimension; the proof for $\gamma$-quantum dimension is identical. Suppose $c>0$ such that $\mathbb{P}\left[\operatorname{dim}_{\mathcal{H}}^{0} \mathcal{N}_{\infty} \geq c\right]>0$. For $r>0$, let $\tau_{r}$ be as in (4.3). We will show that

$$
\mathbb{P}\left[\operatorname{dim}_{\mathcal{H}}^{0} \mathcal{N}_{\tau_{r}} \geq c, \forall r>0\right]=1 .
$$

Since $\mathcal{N}_{\infty}=\bigcup_{r>0} \mathcal{N}_{\tau_{r}}$, by the countable stability of Hausdorff dimension (4.4) implies that a.s. $\operatorname{dim}_{\mathcal{H}}^{0} \mathcal{N}_{\infty} \geq c$, so $\operatorname{dim}_{\mathcal{H}}^{0} \mathcal{N}_{\infty}$ is a.s. equal to a deterministic constant. For every $s>0$, there exists $r>0$ such that $\mathcal{N}_{\tau_{r}} \subset \mathcal{N}_{s} \subset \mathcal{N}_{\infty}$, so by (4.4), a.s. $\mathcal{N}_{s}$ is equal to this same deterministic constant simultaneously for every $s>0$.

It remains to prove (4.4). By our choice of $c$ and the countable stability of Hausdorff dimension, for each $\delta>0$ there exists $r_{0}>0$ and $p>0$ such that $\mathbb{P}\left[\operatorname{dim}_{\mathcal{H}}^{0} \mathcal{N}_{\tau_{r_{0}}} \geq c-\delta\right] \geq p$. By Lemma 4.1, the law of $r^{-1} \mathcal{N}_{\tau_{r}}$ does not depend on $r$. Therefore,

$$
\mathbb{P}\left[\operatorname{dim}_{\mathcal{H}}^{0} \mathcal{N}_{\tau_{r}} \geq c-\delta\right] \geq p, \quad \forall r>0 .
$$

This means that, if we let $\mathcal{T}$ denote the event that $\operatorname{dim}_{\mathcal{H}}^{0} \mathcal{N}_{\tau_{r}} \geq c-\delta$ for arbitrarily small values of $r>0$, then the event $\mathcal{T}$ has probability at least $p$. By Lemma 4.2, the event $\mathcal{T}$ is measurable w.r.t. the tail $\sigma$-algebra $\bigcap_{r>0} \sigma\left(\left.h\right|_{B_{\tau_{r}}(0)}\right)$. Since this $\sigma$-algebra is trivial (see, e.g., [HS18, Lemma 2.2]), the event $\mathcal{T}$ has probability one. Since $\mathcal{N}_{\tau_{r}}$ is increasing in $r$ and $\delta>0$ can be made arbitrarily small, this implies (4.4).

Remark 4.3. Our proof of Theorem 1.11 shows that for every open set $U \subset \mathbb{C}$ containing 0 , a.s. $\operatorname{dim}_{\mathcal{H}}^{0}\left(\mathcal{N}_{s} \cap U\right)=\Delta_{\text {net }}^{0}$ for every $s>0$. We do not rule out the possibility that the dimension of $\mathcal{N}_{s}$ is "concentrated near 0", i.e., there could be open sets $U \subset \mathbb{C}$ which do not contain 0 such that $\mathcal{N}_{s} \cap U \neq \emptyset$ but $\operatorname{dim}_{\mathcal{H}}^{0}\left(\mathcal{N}_{s} \cap U\right)<\Delta_{\text {net }}^{0}$ (we expect, but do not prove, that no such open sets exist). The same is true for the quantum dimension. Similar considerations apply for the other zero-one laws proven in this paper: our proof of Theorem 1.8 does not rule out the possibility that the Euclidean dimension of an LQG geodesic is "concentrated" at the starting point of the geodesic. Likewise, Theorem 1.7 does not rule out the possibility that the $\gamma$-quantum and Euclidean dimensions of $\partial \mathcal{B}_{D_{h}(0, z)}\left(0 ; D_{h}\right)$ are "concentrated" at the point $z$.

\subsection{Scale invariance: defining geodesic rays and metric balls centered at $\infty$}

As we described in the introduction, the dimensions of LQG geodesics and metric ball boundaries are more difficult to study, because they are neither scale invariant nor locally determined by the field. In the rest of this section, we describe how we tackle these two challenges and obtain versions of scale invariance and locality for these fractal that we can use to derive zero-one laws. To get scale invariance, we define "infinite-volume" versions of LQG geodesics and metric ball boundaries whose laws are exactly scale invariant. First, for LQG geodesics, we define an infinite geodesic ray from 0 to $\infty$.

Proposition 4.4. Almost surely, for each $z \in \mathbb{C}$ there exists a (not necessarily unique) infinite geodesic ray $P_{z}^{\infty}$ started from $z$, called a $D_{h}$-geodesic from $z$ to $\infty$. These infinite geodesic rays satisfy the following properties.

(i) For each fixed $z \in \mathbb{C}$, a.s. the geodesic ray $P_{z}^{\infty}$ is unique.

(ii) Almost surely, for each $r>0$ there exists $R>r$ such that for each $z \in B_{r}(0)$, the symmetric difference of $P_{0}^{\infty}$ and $P_{z}^{\infty}$ is contained in $B_{R}(0)$. 
(iii) For each $r>0$,

$$
\left(h(r \cdot)-h_{r}(0), r^{-1} P_{0}^{\infty}\left(r^{\xi Q} e^{\xi h_{r}(0)} \cdot\right)\right) \stackrel{d}{=}\left(h, P_{0}^{\infty}(\cdot)\right)
$$

Proposition 4.4 gives the existence of many one-sided infinite geodesics for $D_{h}$. It is easy to see from confluence that there are no two-sided infinite geodesics for $D_{h}$.

Lemma 4.5. Almost surely, there are no bi-infinite $D_{h}$-geodesics, i.e., there are no paths $P: \mathbb{R} \rightarrow \mathbb{C}$ such that $\left.P\right|_{[s, t]}$ is a $D_{h}$-geodesic for each $s<t$.

Proof. Assume by way of contradiction that there is a bi-infinite $D_{h}$-geodesic with positive probability. Then there exists $q \in(0,1)$ and $R>0$ such that with positive probability, there is a bi-infinite $D_{h^{-}}$ geodesic $P$ which passes through $B_{R}(0)$. By Weyl scaling and the LQG coordinate change formula (Axioms III and IV), if $r>0$ then on the event that $P$ exists, a.s. the path $t \mapsto r^{-1} P\left(e^{\xi h_{r}(0)} t\right)$ is a bi-infinite $D_{h}$-geodesic for the field $h(r \cdot)-h_{r}(0)$. Since $h(r \cdot)-h_{r}(0) \stackrel{d}{=} h$, we get that with probability at least $q$ there is a bi-infinite $D_{h}$-geodesic for $h$ which passes through $B_{R r}(0)$. Since this holds for every $r>0$, we get that with probability at least $q$ there is a (random) sequence $r^{n} \rightarrow 0$

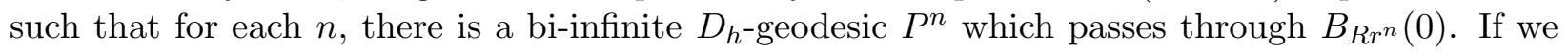
parametrize $P^{n}$ so that $P^{n}(0) \in B_{R r^{n}}(0)$, then by the Arzéla-Ascoli theorem, the paths $P^{n}$ admit a subsequential limit with respect to the local uniform topology for paths $\mathbb{R} \rightarrow \mathbb{C}$. The limiting path is a bi-infinite $D_{h}$-geodesic which passes through 0 . But, a.s. there is no bi-infinite $D_{h}$-geodesic which passes through 0 due to confluence of geodesics [GM20a, Theorem 1.3].

For the proof of Proposition 4.4 we need the following variant of Theorem 1.2.

Lemma 4.6. For each $p \in(0,1)$, there exists $A=A(p, \gamma)>1$ such that for each fixed $r>0$, it holds with probability at least $p$ that the following is true. There is a point $z_{0} \in B_{A r}(0) \backslash B_{r}(0)$ such that every $D_{h}$-geodesic from a point of $B_{r}(0)$ to a point of $\mathbb{C} \backslash B_{A r}(0)$ passes through $z_{0}$.

Proof. By Theorem 1.2 applied with $U=\mathbb{D}$, there exists $A=A(p, \gamma)>1$ such that the statement of the lemma holds with this choice of $A$ and with $r=1 / A$. By Weyl scaling (Axiom III), for each fixed $r>1$, the occurrence event in the lemma statement does not depend on the choice of additive constant for $h$. By the LQG coordinate change formula (Axiom IV) and the scale invariance of the law of $h$, modulo additive constant, we see that for a fixed choice of $A$, the probability of the event in the lemma statement does not depend on $r$.

Proof of Proposition 4.4. By Lemma 4.6, for each $p \in(0,1)$ there exists $A=A(p, \gamma)>1$ such that for each $k \in \mathbb{N}$, we have $\mathbb{P}\left[E_{k}\right] \geq p$, where $E_{k}$ is the event that the following is true. There is a

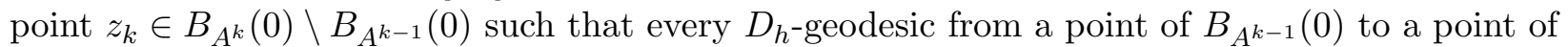
$\mathbb{C} \backslash B_{A^{k}}(0)$ passes through $z_{k}$. With probability at least $p$, the event $E_{k}$ occurs for infinitely many $k \in \mathbb{N}$.

Since $p$ can be made arbitrarily close to 1 , we get that a.s. the following is true. There is a sequence of positive radii $R_{n} \rightarrow \infty$ and points $z_{n} \in B_{R_{n}}(0) \backslash B_{R_{n-1}}(0)$ such that every $D_{h^{-} \text {geodesic }}$ from a point of $B_{R_{n-1}}(0)$ to a point of $\mathbb{C} \backslash B_{R_{n}}(0)$ passes through $z_{n}$. We set $z_{0}:=0$.

We claim that for each $n \in \mathbb{N}$, there is a unique $D_{h}$-geodesic from $z_{n-1}$ to $z_{n}$. To see this, let $q \in \mathbb{Q}^{2} \backslash B_{R_{n}}(0)$. Then a.s. the $D_{h}$-geodesic $P_{q}$ from 0 to $q$ is unique and this $D_{h}$-geodesic must pass through both $z_{n-1}$ and $z_{n}$. If there were more than one $D_{h}$-geodesic from $z_{n-1}$ to $z_{n}$, then we could replace the segment of $P_{q}$ between $z_{n-1}$ and $z_{n}$ by one of these geodesics to get a contradiction to the uniqueness of $P_{q}$.

In particular, for each $n \in \mathbb{N}$ the $D_{h}$-geodesic from 0 to $z_{n}$ is unique. Moreover, the $D_{h}$-geodesic from 0 to $z_{n-1}$ is the sub-path of the $D_{h}$-geodesic from 0 to $z_{n}$ between 0 and $z_{n-1}$. By sending 


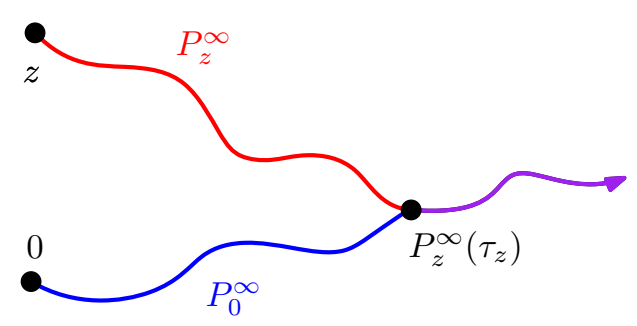

Figure 6: The infinite $D_{h}$-geodesic ray started from 0 (resp. $z$ ) is the union of the blue and purple (resp. red and purple) curves. The point $z$ belongs to $\mathcal{B}_{0}^{\infty}$ if and only if the $D_{h}$-length of the red geodesic segment is smaller than or equal to the length of the blue geodesic segment.

$n \rightarrow \infty$, we get that a.s. there is a unique infinite geodesic ray $P_{0}^{\infty}$ from 0 to $\infty$. By the translation invariance of the law of $h$, modulo additive constant, this shows that for each fixed $z \in \mathbb{C}$ there is a.s. a unique infinite geodesic ray from $z$ to $\infty$, i.e., assertion (i) holds.

To construct infinite geodesic rays for all possible starting points simultaneously, consider $z \in \mathbb{C}$ and let $n \in \mathbb{N}$ be chosen so that $z \in B_{R_{n-1}}(0)$. There is a $D_{h}$-geodesic $P$ from $z$ to $z_{n+1}$, which must pass through $z_{n}$. In particular, $P$ coincides with the unique $D_{h}$-geodesic from $z_{n}$ to $z_{n+1}$ between the times when it hits $z_{n}$ and $z_{n+1}$. From this, it follows that the concatenation of $P$, stopped upon hitting $z_{n}$, with the $D_{h}$-geodesic ray from $z_{n}$ to $\infty$ is a $D_{h}$-geodesic ray from $z$ to $\infty$.

We next prove assertion (ii). Given $r>0$, choose $n \in \mathbb{N}$ such that $r \leq R_{n-1}$ and let $R:=R_{n}$. A $D_{h}$-geodesic from a point of $B_{r}(0)$ to $\infty$ stopped when it first hits $\mathbb{C} \backslash B_{R}(0)$ is a $D_{h}$-geodesic from a point of $B_{r}(0)$ to a point of $\mathbb{C} \backslash B_{R}(0)$. By the definition of the $R_{n}$ 's, every $D_{h}$-geodesic from a point of $B_{r}(0)$ to $\infty$ must pass through $z_{n}$. Similarly, each such $D_{h}$-geodesic must hit $z_{N}$ for each $N \geq n$. By the uniqueness of the $D_{h}$-geodesic from $z_{N}$ to $z_{N+1}$, each such $D_{h}$-geodesic must coincide with $P_{0}^{\infty}$ after hitting $z_{n}$, so must coincide with $P_{0}^{\infty}$ after its first exit time from $B_{R}(0)$.

Finally, we prove assertion (iii). By Axioms III and IV, applied in the same manner as in Lemma 4.1, we get that $r^{-1} P_{0}^{\infty}\left(r^{\xi Q} e^{\xi h_{r}(0)} \cdot\right)$ is an infinite geodesic ray started from 0 for $D_{h(r \cdot)-h_{r}(0)}$. Since such an infinite geodesic ray is unique (assertion (i)) and $h(r \cdot)-h_{r}(0) \stackrel{d}{=} h$, we obtain (4.6).

For LQG metric ball boundaries, we define a "metric ball started from $\infty$ and grown until it hits $0 "$ as the limit of $\mathcal{B}_{D_{h}(0, w)}\left(w ; D_{h}\right)$ as $w \rightarrow \infty$.

Proposition 4.7. There is a random unbounded set $\mathcal{B}_{0}^{\infty}=" \mathcal{B}_{D_{h}(0, \infty)}\left(\infty ; D_{h}\right)$ " $\subset \mathbb{C}$ such that the balls $\mathcal{B}_{D_{h}(0, w)}\left(w ; D_{h}\right)$ converge to $\mathcal{B}_{0}^{\infty}$ as $w \rightarrow \infty$ in the following sense. Almost surely, for each $r>0$, there exists $R>r$ such that

$$
\mathcal{B}_{D_{h}(0, w)}\left(w ; D_{h}\right) \cap B_{r}(0)=\mathcal{B}_{0}^{\infty} \cap B_{r}(0), \quad \forall w \in \mathbb{C} \backslash B_{R}(0) .
$$

Furthermore, $\mathcal{B}_{0}^{\infty}$ is a.s. determined by $h$ viewed modulo additive constant and the law of $\mathcal{B}_{0}^{\infty}$ is scale invariant in the sense that

$$
\left(h, \mathcal{B}_{0}^{\infty}\right) \stackrel{d}{=}\left(h(r \cdot)-h_{r}(0), r^{-1} \mathcal{B}_{0}^{\infty}\right), \quad \forall r>0 .
$$

Due to the translation invariance of the law of $h$, modulo additive constant, Proposition 4.7 allows us to define the ball $\mathcal{B}_{z}^{\infty}=$ " $\mathcal{B}_{D_{h}(z, \infty)}\left(\infty ; D_{h}\right)$ " for each $z \in \mathbb{C}$. In particular, $\mathcal{B}_{z}^{\infty}$ is constructed from $h(\cdot+z)$ in the same manner that $\mathcal{B}_{0}^{\infty}$ is constructed from $h$.

Proof of Proposition 4.7. For $z \in \mathbb{C}$, let $P_{z}^{\infty}$ be a $D_{h}$-geodesic from $z$ to $\infty$, as in Proposition 4.4 (chosen in an arbitrary manner if there is more than one such $D_{h}$-geodesic). By Proposition $4.4, P_{0}^{\infty}$ 
is a.s. unique and each $P_{z}^{\infty}$ merges into $P_{0}^{\infty}$ at some finite time. Let $\tau_{z}:=\inf \left\{t \geq 0: P_{z}^{\infty}(t) \in P_{0}^{\infty}\right\}$ and define

$$
\mathcal{B}_{0}^{\infty}:=\left\{z \in \mathbb{C}: \tau_{z} \leq D_{h}\left(0, P_{z}^{\infty}\left(\tau_{z}\right)\right)\right\} .
$$

In other words, $\mathcal{B}_{0}^{\infty}$ is the set of $z \in \mathbb{C}$ for which the segment of $P_{z}^{\infty}$ before it first hits $P_{0}^{\infty}$ is shorter than the segment of $P_{0}^{\infty}$ before it reaches $P_{z}^{\infty}\left(\tau_{z}\right)$. See Figure 6 for an illustration. We note that $P_{z}^{\infty}$ merges into $P_{0}^{\infty}$ at time $\tau_{z}$, i.e., $P_{z}^{\infty}\left(\left[\tau_{z}, \infty\right)\right) \subset P_{0}^{\infty}$ : if not, then we could find two distinct $D_{h}$-geodesic rays from 0 to $\infty$ by replacing a segment of $P_{0}^{\infty}$ by a segment of $P_{z}^{\infty}$, which would contradict the uniqueness of $P_{0}^{\infty}$.

To make sure that $\mathcal{B}_{0}^{\infty}$ is well-defined, we need to check that the definition of $\mathcal{B}_{0}^{\infty}$ does not depend on the choice of $P_{z}^{\infty}$ in the case when the geodesic from $z$ to $\infty$ is not unique. Indeed, suppose $P_{z}^{\infty}$ and $\widetilde{P}_{z}^{\infty}$ are two $D_{h}$-geodesics from $z$ to $\infty$ and let $\widetilde{\tau}_{z}$ be defined as above with $\widetilde{P}_{z}^{\infty}$ in place of $P_{z}^{\infty}$. By re-labeling, we can assume without loss of generality that $D_{h}\left(0, \widetilde{P}_{z}^{\infty}\left(\widetilde{\tau}_{z}\right)\right) \leq D_{h}\left(0, P_{z}^{\infty}\left(\tau_{z}\right)\right)$. Then $\left.P_{z}^{\infty}\right|_{\left[0, \tau_{z}\right]}$ and the concatenation of $\left.\widetilde{P}_{z}^{\infty}\right|_{\left[0, \widetilde{\tau}_{z}\right]}$ with $\left.P_{0}^{\infty}\right|_{\left[D_{h}\left(0, \widetilde{P}_{z}^{\infty}\left(\widetilde{\tau}_{z}\right)\right), D_{h}\left(0, P_{z}^{\infty}\left(\tau_{z}\right)\right)\right]}$ are each $D_{h}$-geodesics from 0 to $P_{z}^{\infty}\left(\tau_{z}\right)$, so their lengths agree, i.e.,

$$
\tau_{z}=\widetilde{\tau}_{z}+D_{h}\left(0, P_{z}^{\infty}\left(\tau_{z}\right)\right)-D_{h}\left(0, \widetilde{P}_{z}^{\infty}\left(\widetilde{\tau}_{z}\right)\right)
$$

This shows that the definition of (4.9) is unaffected by replacing $P_{z}^{\infty}$ with $\widetilde{P}_{z}^{\infty}$.

We now check the convergence property (4.7). By Lemma 4.6, a.s. for each $r>0$ there exists $R>0$ and a point $Z_{r} \in B_{R}(0) \backslash B_{r}(0)$ such that every $D_{h}$-geodesic from a point of $B_{r}(0)$ to a point of $\mathbb{C} \backslash B_{R}(0)$ passes through $Z_{r}$. Hence

$$
D_{h}(z, w)=D_{h}\left(z, Z_{r}\right)+D_{h}\left(Z_{r}, w\right), \quad \forall z \in B_{r}(0), \quad \forall w \in \mathbb{C} \backslash B_{R}(0) .
$$

Furthermore, for each $z \in B_{r}(0)$, the $D_{h}$-geodesic $P_{0}^{\infty}$ hits $P_{z}^{\infty}\left(\tau_{z}\right)$ before hitting $Z_{r}$ and hence

$$
D_{h}\left(0, Z_{r}\right)=D_{h}\left(0, P_{z}^{\infty}\left(\tau_{z}\right)\right)+D_{h}\left(P_{z}^{\infty}\left(\tau_{z}\right), Z_{r}\right) \quad \text { and } \quad D_{h}\left(z, Z_{r}\right)=\tau_{z}+D_{h}\left(P_{z}^{\infty}\left(\tau_{z}\right), Z_{r}\right) .
$$

By subtracting the two equations in (4.11) then applying (4.10) once for $z$ and once with 0 in place of $z$, we obtain that for each $w \in \mathbb{C} \backslash B_{R}(0)$,

$$
\tau_{z}-D_{h}\left(0, P_{z}^{\infty}\left(\tau_{z}\right)\right)=D_{h}\left(z, Z_{r}\right)-D_{h}\left(0, Z_{r}\right)=D_{h}(z, w)-D_{h}(0, w) .
$$

Recalling (4.9), we now get (4.7).

Due to Weyl scaling (Axiom III), adding a constant to $h$ causes us to multiply both $\tau_{z}$ and $D_{h}\left(0, P_{z}^{\infty}\left(\tau_{z}\right)\right)$ in (4.9) by the factor $e^{\xi C}$. It follows that adding a constant to $h$ does not affect the definition of $\mathcal{B}_{0}^{\infty}$, so $\mathcal{B}_{0}^{\infty}$ is a.s. determined by $h$ modulo additive constant.

It remains to prove the scaling relation (4.8). From (4.9) and Weyl scaling (Axiom III), it is immediate that the definition of $\mathcal{B}_{0}^{\infty}$ is unaffected by adding a constant to $h$. From this, Axiom IV, and the scale invariance of the law of $h$ modulo additive constant, we get (4.8).

\subsection{Locality: applying the strong confluence property}

We now apply Theorem 1.2 to construct an event of positive probability on which LQG geodesics and metric ball boundaries are in some sense locally determined by $h$.

Lemma 4.8. For each $p \in(0,1)$, there exists $A=A(p, \gamma)>1$ such that for each $z \in \mathbb{C}$ and $r>0$, there is a random point $Z_{r}(z) \in B_{A r}(z) \backslash B_{r}(z)$ and an event $E_{r}(z)$, both of which are a.s. given by measurable functions of $\left.h\right|_{B_{A^{2}}(z) \backslash B_{r / A}(z)}$ viewed modulo additive constant, such that the following is true. We have $\mathbb{P}\left[E_{r}(z)\right] \geq p$. Moreover, the metric $D_{h}$ has the following properties on the event $E_{r}(z)$ 
(i) Each $D_{h}$-geodesic from a point of $B_{r}(z)$ to a point of $\mathbb{C} \backslash B_{A r}(z)$ passes through $Z_{r}(z)$.

(ii) Each $D_{h}$-geodesic between two points of $\overline{B_{A r}(z)}$ is contained in $B_{A^{2} r}(z)$.

(iii) Each $D_{h}$-geodesic between two points of $\mathbb{C} \backslash B_{r}(z)$ is contained in $\mathbb{C} \backslash \overline{B_{r / A}(z)}$. More strongly, there is a path $\pi \subset B_{r}(z) \backslash \overline{B_{r / A}(z)}$ such that

$$
\left(D_{h} \text {-length of } \pi\right)<D_{h}\left(\pi, B_{r / A}(z)\right) \text {. }
$$

Proof. Step 1: defining the event. Let $A>1$ to be chosen later and for $z \in \mathbb{C}$, let $E_{r}(z)$ be the event that the following is true.

1. There is a point $Z \in B_{A r}(z) \backslash B_{r}(z)$ such that every $D_{h}\left(\cdot, \cdot ; B_{A^{2} r}(z) \backslash \overline{B_{r / A}(z)}\right)$-geodesic from a point of $\partial B_{r}(z)$ to a point of $\partial B_{A r}(z)$ passes through $Z$.

2. There is a number $\rho \in\left[A r, A^{2} r / 4\right]$ with the following property. There is path in the annulus $B_{2 \rho}(z) \backslash B_{\rho}(z)$ which disconnects the inner and outer boundaries of the annulus and whose $D_{h}$-length is at most $\frac{1}{2} D_{h}\left(\partial B_{2 \rho}(z), \partial B_{4 \rho}(z)\right)$.

3. There is a number $\rho^{\prime} \in[r / A, r / 4]$ with the following property. There is path $\pi$ in the annulus $B_{4 \rho^{\prime}}(z) \backslash B_{2 \rho^{\prime}}(z)$ which disconnects the inner and outer boundaries of the annulus and whose $D_{h^{-}}$length is at most $\frac{1}{2} D_{h}\left(\partial B_{\rho^{\prime}}(z), \partial B_{2 \rho^{\prime}}(z)\right)$.

By the locality of the metric (Axiom II), we have $E_{r}(z) \in \sigma\left(\left.h\right|_{B_{A^{2} r}(z) \backslash B_{r / A}(z)}\right)$ (this is why we use the internal metric on $B_{A^{2} r}(z)$ in condition 1). It is easily seen from Axiom III that adding a constant to $h$ does not affect the occurrence of $E_{r}(z)$, so $E_{r}(z)$ is determined by $\left.h\right|_{B_{A^{2} r}(z) \backslash B_{r / A}(z)}$ viewed modulo additive constant. On $E_{r}(z)$, we can choose $Z_{r}(z) \in B_{A r}(z) \backslash B_{r}(z)$ in a manner depending only on $\left.h\right|_{B_{A^{2} r}(z) \backslash B_{r / A}(z)}$ viewed modulo additive constant, such that condition 1 in the definition of $E_{r}(z)$ occurs with $Z=Z_{r}(z)$. On the complement of $E_{r}(z)$, we arbitrarily define $Z_{r}(z):=z+(r, 0)$. Then the pair $\left(E_{r}(z), Z_{r}(z)\right)$ satisfies the measurability condition in the lemma statement.

Step 2: properties of the event. We now assume that $E_{r}(z)$ occurs and check the three numbered properties in the lemma statement. We start with property (ii). Let $\rho$ be as in condition 2 in the definition of $E_{r}(z)$ and let $\pi$ be the path in $B_{2 \rho}(z) \backslash B_{\rho}(z)$ as in that condition. Suppose $P:[0, T] \rightarrow \mathbb{C}$ is a path between two points of $\overline{B_{A r}(z)}$ which exits $B_{A^{2} r}(z)$. We claim that $P$ is not a $D_{h}$-geodesic. Indeed, since each of $\pi$ and $B_{4 \rho}(z) \backslash B_{2 \rho}(z)$ disconnects $\overline{B_{A r}(z)}$ from $\mathbb{C} \backslash B_{A^{2} r}(z)$ there must be times $0<s<t<T$ with the following properties. We have $P(s), P(t) \in \pi$ and $P$ crosses between the inner and outer boundaries of the annulus $B_{4 \rho}(z) \backslash B_{2 \rho}(z)$ between time $s$ and

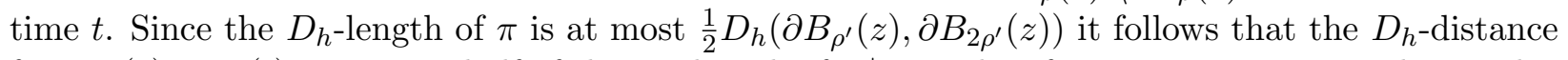
from $P(s)$ to $P(t)$ is at most half of the $D_{h}$-length of $\left.P\right|_{[s, t]}$. Therefore $P$ is not a $D_{h}$-geodesic. This gives property (ii). We similarly obtain property (iii) from condition 3 in the definition of $E_{r}(z)$, with the path $\pi$ as in condition 3.

We now check property (i). The combination of properties (ii) and (iii) of $E_{r}(z)$ tells us that every $D_{h}$-geodesic between points of $\overline{B_{A r}(z) \backslash B_{r}(z)}$ is contained in $B_{A^{2} r}(z) \backslash \overline{B_{r / A}(z)}$. This implies that the set of $D_{h}\left(\cdot, \cdot ; B_{A^{2} r}(z) \backslash \overline{B_{r / A}(z)}\right)$-geodesics between any two points of $\overline{B_{A r}(z) \backslash B_{r}(z)}$ is the same as the set of $D_{h}$-geodesics between these two points. Consequently, condition 1 in the definition of $E_{r}(z)$ (together with the definition of $Z_{r}(z)$ ) implies that every $D_{h}$-geodesic from a

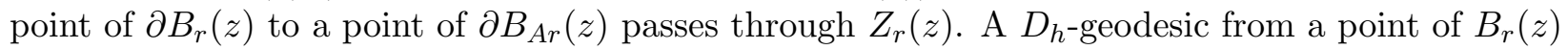


to a point of $\mathbb{C} \backslash B_{A r}(z)$ has a sub-segment which is a $D_{h}$-geodesic from a point of $\partial B_{r}(z)$ to a point of $\partial B_{A r}(z)$, so any such $D_{h}$-geodesic must also pass through $Z_{r}(z)$. This gives property (i).

Step 3: estimating the probability of $E_{r}(z)$. It remains to show that we can choose $A$ in such a way that $\mathbb{P}\left[E_{r}(z)\right] \geq p$ for each $z \in \mathbb{C}$ and $r>0$. By the scale and translation invariance of the law of $h$, modulo additive constant, and Axioms III and IV, $\mathbb{P}\left[E_{r}(z)\right]$ does not depend on $z$ or $r$. Hence it suffices to choose $A$ so that $\left.\mathbb{P}\left[E_{1}(0)\right)\right] \geq p$.

We first deal with condition 2 as follows. For $\rho>0$, let $G_{\rho}$ be the event that there is a path in $B_{2 \rho}(0) \backslash B_{\rho}(0)$ which disconnects the inner and outer boundaries of the annulus and whose $D_{h}$-length is at most $\frac{1}{2} D_{h}\left(\partial B_{2 \rho}(0), \partial B_{4 \rho}(0)\right)$. By the scale invariance of the law of $h$, modulo additive constant, together with Axioms III and IV (Weyl scaling and coordinate change), we see that $\mathbb{P}\left[G_{\rho}\right]$ does not depend on $\rho$. By Axiom II we see that $G_{\rho}$ is a.s. determined by $h_{B_{4 \rho}(0) \backslash B_{\rho}(0)}$ viewed modulo additive constant. By an easy absolute continuity argument (see, e.g., [Gwy20a, Lemma 6.1]) we have $q:=\mathbb{P}\left[G_{1}\right]>0$. Since $\mathbb{P}\left[G_{\rho}\right]=q$ for every $\rho>0$ and the tail $\sigma$-algebra $\bigcap_{\rho>1} \sigma\left(\left.h\right|_{\mathbb{C} \backslash B_{\rho}(0)}\right)$ is trivial, it follows that a.s. $G_{\rho}$ occurs for infinitely many positive integer values of $\rho$.

Therefore, we can choose $A_{0}>4$ such that with probability at least $1-p / 3$, the event $G_{\rho}$ occurs for at least one value of $\rho$ in $\left[1, A_{0} / 4\right]$. By scale invariance, if $A>0$ it also holds with probability at least $1-p / 3$ that $G_{\rho}$ occurs for at least one value of $\rho$ in $\left[A, A_{0} A / 4\right]$. Hence if $A \geq A_{0}$ then condition 2 in the definition of $E_{1}(0)$ occurs with probability at least $1-(1-p) / 3$. By an identical argument, we see that after possibly increasing $A_{0}$, for any $A \geq A_{0}$ it holds with probability at least $1-2(1-p) / 3$ that conditions 2 and 3 in the definition of $E_{1}(0)$ both occur.

By Lemma 4.6 (with $r=1$ ) combined with the preceding paragraph, there exists $A=A(p, \gamma) \geq$ $A_{0}$ such that with probability at least $p$, conditions 1 and 3 in the definition of $E_{1}(0)$ both occur and also there is a point $Z \in B_{A}(0) \backslash B_{1}(0)$ such that every $D_{h}$-geodesic from a point of $\partial B_{1}(0)$ to a point of $\partial B_{A}(0)$ passes through $Z$. As explained in step 2, if conditions 2 and 3 in the definition of $E_{1}(0)$ both occur then the set of $D_{h}\left(\cdot, \cdot ; B_{A^{2}}(0) \backslash \overline{B_{1 / A}(0)}\right)$-geodesics between any two points of $B_{A}(0) \backslash \overline{B_{1}(0)}$ is the same as a $D_{h}$-geodesics between these two points. We therefore have $\mathbb{P}\left[E_{1}(0)\right] \geq p$, as required.

\section{Zero-one law for LQG geodesics}

In this section, we prove a zero-one law for LQG geodesics (Theorem 1.8). We begin by proving a zero-one law for the Euclidean dimension of the infinite geodesic ray $P_{0}^{\infty}$ from Proposition 4.4. This case is easier than the case of general geodesics since the law of $P_{0}^{\infty}$ is scale invariant.

Proposition 5.1. Let $P_{0}^{\infty}$ be the geodesic ray from 0 to $\infty$ as in Proposition 4.4. There is a deterministic constant $\Delta_{\text {geo }}>0$ such that the random variable $\operatorname{dim}_{\mathcal{H}}^{0} P_{0}^{\infty}$ is a.s. equal to $\Delta_{\text {geo }}$. Moreover, a.s. $\operatorname{dim}_{\mathcal{H}}^{0} P \geq \Delta_{\text {geo }}$ for each $D_{h}$-geodesic $P$ from 0 to a point of $\mathbb{C} \backslash\{0\}$.

Proof. The proof is similar to that of Theorem 1.11. Let $c>0$ such that $\mathbb{P}\left[\operatorname{dim}_{\mathcal{H}}^{0} P_{0}^{\infty} \geq c\right]>0$. We claim that a.s. for each $D_{h}$-geodesic $P$ from 0 to a point of $\mathbb{C} \backslash\{0\}$,

$$
\operatorname{dim}_{\mathcal{H}}^{0}\left(P \cap B_{r}(0)\right) \geq c, \quad \forall r>0 .
$$

Applying (5.1) with $P=P_{0}^{\infty}$ shows that $\operatorname{dim}_{\mathcal{H}}^{0} P_{0}^{\infty}$ is a.s. equal to a deterministic constant, and then applying (5.1) for an arbitrary choice of $P$ shows that a.s. $\operatorname{dim}_{\mathcal{H}}^{0} P$ is bounded below by this constant for every $D_{h}$-geodesic $P$ from 0 to a point of $\mathbb{C} \backslash\{0\}$.

To prove (5.1), we first use the countable stability of Hausdorff dimension to get that for each $\delta>0$, there exists $r_{0}>0$ and $p \in(0,1)$ such that $\mathbb{P}\left[\operatorname{dim}_{\mathcal{H}}^{0}\left(P_{0}^{\infty} \cap B_{r_{0}}(0)\right) \geq c-\delta\right] \geq p$. By the scale 
invariance of the law of $h$, modulo additive constant, and (4.6),

$$
\mathbb{P}\left[\operatorname{dim}_{\mathcal{H}}^{0}\left(P_{0}^{\infty} \cap B_{r}(0)\right) \geq c-\delta\right] \geq p, \quad \forall r>0 .
$$

Obviously, $\operatorname{dim}_{\mathcal{H}}^{0}\left(P_{0}^{\infty} \cap B_{r}(0)\right)$ is increasing in $r$, so by (5.2),

$$
\mathbb{P}\left[\exists \text { arbitrarily small values of } r>0 \text { such that } \operatorname{dim}_{\mathcal{H}}^{0}\left(P_{0}^{\infty} \cap B_{r}(0)\right) \geq c-\delta\right] \geq p \text {. }
$$

By confluence of geodesics started from 0 [GM20a, Theorem 1.3] (plus the fact that the LQG metric induces the same topology as the Euclidean metric), a.s. for each $r>0$ there exists $r^{\prime} \in(0, r)$ such

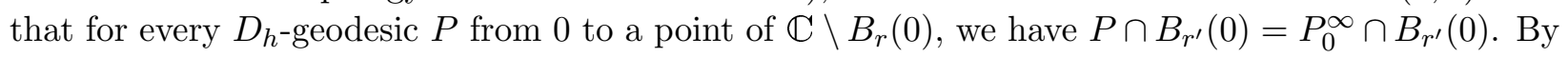
combining this with (5.3), we obtain that for each $r_{2}>r_{1}>0$,

$$
\mathbb{P}\left[\operatorname{dim}_{\mathcal{H}}^{0}\left(P \cap B_{r_{1}}(0)\right) \geq c-\delta, \forall \text { geodesic } P \text { from } 0 \text { to a point of } \mathbb{C} \backslash B_{r_{2}}(0)\right] \geq p .
$$

We will now deduce (5.1) from (5.4) together with tail triviality considerations. To do this we will use Lemma 4.8 for convenience, but we do not need the full force of the lemma here (we do need all of the conditions from Lemma 4.8 to treat the case of the metric ball boundary, however). Let $A=A(1-p / 2, \gamma)$ be as in Lemma 4.8 with $1-p / 2$ in place of $p$. For $r>0$, let $E_{r}=E_{r}(0)$ be the event from that lemma, so that $E_{r} \in \sigma\left(\left.h\right|_{B_{A^{2} r}(0)}\right)$ and $\mathbb{P}\left[E_{r}\right] \geq 1-p / 2$. Let $G_{r}$ be the intersection of $E_{r}$ with the event that every $D_{h}$-geodesic $P$ from 0 to a point of $\partial B_{A r}(0)$ satisfies $\operatorname{dim}_{\mathcal{H}}^{0}\left(P \cap B_{r}(0)\right) \geq c-\delta$. By (5.4) (with $r_{1}=r$ and $\left.r_{2}=A r\right)$,

$$
\mathbb{P}\left[G_{r}\right] \geq p / 2, \quad \forall r>0 .
$$

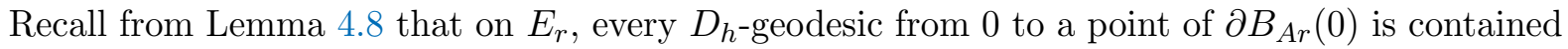
in $B_{A^{2} r}(0)$, so on $E_{r}$ the set of such $D_{h}$-geodesics is the same as the set of $D_{h}\left(\cdot, \cdot ; B_{A^{2} r}(0)\right)$-geodesics from 0 to points of $\partial B_{A r}(0)$. Since $E_{r} \in \sigma\left(\left.h\right|_{B_{A^{2} r}(0)}\right)$ and by Axiom II (locality), we get that $G_{r} \in \sigma\left(\left.h\right|_{B_{A^{2} r}(0)}\right)$.

By (5.5), it holds with probability at least $p / 2$ that there are arbitrarily small values of $r>0$ for which $G_{r}$ occurs. Since $G_{r} \in \sigma\left(\left.h\right|_{B_{A^{2} r}(0)}\right)$ and the tail $\sigma$-algebra $\bigcap_{r>0} \sigma\left(\left.h\right|_{B_{A^{2} r}(0)}\right)$ is trivial [HS18, Lemma 2.2], this implies that in fact a.s. $G_{r}$ occurs for arbitrarily small values of $r>0$. Henceforth assume that we are working on the (full probability) event that this is the case.

For each $D_{h}$-geodesic $P$ from 0 to a point $z \in \mathbb{C} \backslash\{0\}$ and each $r \in(0,|z| / A)$ there is a segment of $P$ which is a $D_{h}$-geodesic from 0 to a point of $\partial B_{A r}(0)$. If $G_{r}$ occurs, then this segment of $P$ has Euclidean dimension at least $c-\delta$. From the preceding paragraph, we therefore get that $\operatorname{dim}_{\mathcal{H}}^{0}\left(P \cap B_{r}(0)\right) \geq c-\delta$ for arbitrarily small values of $r>0$. Since $\delta>0$ can be made arbitrarily small, this implies (5.1).

We now want to argue that in fact the Euclidean dimension of any $D_{h}$-geodesic started from 0 is bounded above by the constant $\Delta_{\text {geo }}$ from Proposition 5.1. The idea of the proof is that if we see $\mathcal{B}_{s}^{\bullet}$ and $\left.h\right|_{\mathcal{B}_{s}^{\bullet}}$ for some $s>0$, then we cannot tell which $D_{h}$-geodesic from 0 to $\partial \mathcal{B}_{s}^{\bullet}$ is equal to $\left.P_{0}^{\infty}\right|_{[0, s]}$, so all of these $D_{h}$-geodesics must have dimension at most $\Delta_{\text {geo }}$. The following lemma makes precise the idea that $\mathcal{B}_{s}^{\bullet}$ and $\left.h\right|_{\mathcal{B}_{s}^{\bullet}}$ do not determine which point of $\partial \mathcal{B}_{s}^{\bullet}$ is hit by $P_{0}^{\infty}$.

Lemma 5.2. Fix $s>0$ and $\delta>0$. Let $I$ be a non-trivial arc of $\partial \mathcal{B}_{s}^{\bullet}$ chosen in a $\sigma\left(\mathcal{B}_{s}^{\bullet},\left.h\right|_{\mathcal{B}_{s}^{\bullet}}\right)$ measurable manner (recall from Lemma 2.4that $\partial \mathcal{B}_{s}^{\bullet}$ is a Jordan curve). Almost surely, it holds with positive conditional probability given $\sigma\left(\mathcal{B}_{s}^{\bullet},\left.h\right|_{\mathcal{B}_{s}^{\bullet}}\right)$ that the following is true. For each $w \in$ $\mathbb{C} \backslash B_{\delta}\left(\mathcal{B}_{s}^{\bullet}\right)$, every $D_{h}$-geodesic from 0 to $w$ passes through $I$ (recall that $B_{\delta}(\cdot)$ denotes the Euclidean $\delta$-neighborhood). 


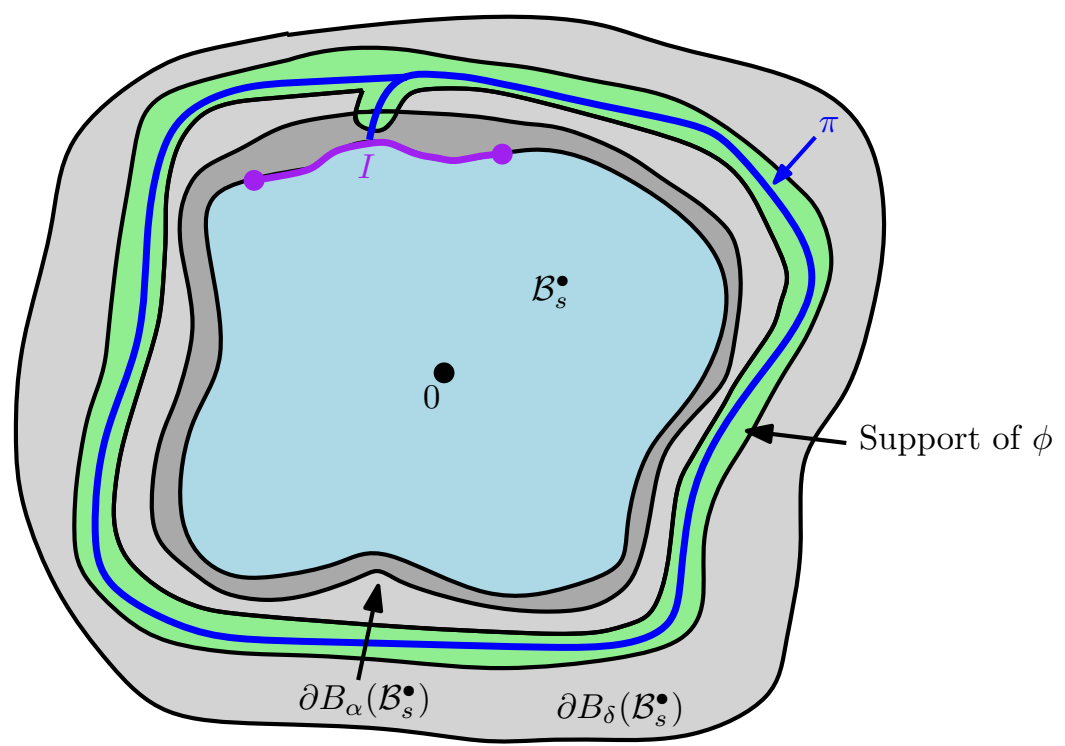

Figure 7: Illustration of the proof of Lemma 5.2. We condition on $\left(\mathcal{B}_{s}^{\bullet},\left.h\right|_{\mathcal{B}_{s}^{\bullet}}\right)$ and consider the field $\widetilde{h}=h-C \phi$ where $\phi$ is a smooth bump function supported in the light green region and $C$ is large. Then the conditional laws of $h$ and $\widetilde{h}$ given $\left(\mathcal{B}_{s}^{\bullet},\left.h\right|_{\mathcal{B}_{s}^{\bullet}}\right)$ are mutually absolutely continuous. On the other hand, if we make the constant $C$ large enough and we make the support of $\phi$ sufficiently close to $I$, then the $D_{\widetilde{h}}$-distance from any point $z$ on the blue path $\pi$ to $I$ is smaller than the $D_{\widetilde{h}}$-distance from $z$ to any point of $\partial \mathcal{B}_{s}^{\bullet} \backslash I$. Since every path from 0 to a point outside of $B_{\delta}\left(\mathcal{B}_{s}^{\bullet}\right)$ passes through $\pi$, this forces every $D_{\tilde{h}^{-}}$geodesic from 0 to a point outside of $B_{\delta}\left(\mathcal{B}_{s}^{\bullet}\right)$ to pass through $I$ (otherwise, we could replace it by a shorter path which did pass through $I$ ).

Proof. To lighten notation, let $\mathcal{F}:=\sigma\left(\mathcal{B}_{s}^{\bullet},\left.h\right|_{\mathcal{B}_{s}^{\bullet}}\right)$. Throughout the proof, we will condition on $\mathcal{F}$ and all choices are required to be made in a $\mathcal{F}$-measurable manner. See Figure 7 for an illustration of the argument.

We first choose (in a $\mathcal{F}$-measurable manner) a path $\pi$ in the annular region $\left(B_{\delta}\left(\mathcal{B}_{s}^{\bullet}\right) \backslash \mathcal{B}_{s}^{\bullet}\right) \cup I$ which starts from a point of $I$, lies at positive Euclidean distance from $\partial \mathcal{B}_{s}^{\bullet} \backslash I$, and disconnects $\mathcal{B}_{s}^{\bullet}$ from $\partial B_{\delta}\left(\mathcal{B}_{s}^{\bullet}\right)$. Let $\varepsilon>0$ be small enough so that $\pi$ lies at Euclidean distance at least $100 \varepsilon$ from each of $\partial \mathcal{B}_{s}^{\bullet} \backslash I$ and $\partial B_{\delta}\left(\mathcal{B}_{s}^{\bullet}\right)$.

Since $D_{h}$ a.s. induces the Euclidean topology, we can find small enough $\zeta, \alpha \in(0,(\varepsilon \wedge \delta) / 100)$ and a large enough $A>1$ (all random and $\mathcal{F}$-measurable) such that the following is true with conditional probability at least $1 / 2$ given $\mathcal{F}$.

1. The $D_{h}$-distance from the $2 \varepsilon$-neighborhood $B_{2 \varepsilon}(\pi)$ to each of $\partial \mathcal{B}_{s}^{\bullet} \backslash I$ and $\partial B_{\delta}\left(\mathcal{B}_{s}^{\bullet}\right)$ is at least $100 \zeta$.

2. The $D_{h}$-distance from each point of $B_{2 \alpha}\left(\mathcal{B}_{s}^{\bullet}\right)$ to $\mathcal{B}_{s}^{\bullet}$ is at most $\zeta$.

3. For each $z \in \pi \backslash B_{2 \alpha}\left(\mathcal{B}_{s}^{\bullet}\right)$, there is a path from $z$ to a point of $B_{2 \alpha}\left(\mathcal{B}_{s}^{\bullet}\right)$ which is contained in $B_{\varepsilon}(\pi) \backslash B_{2 \alpha}\left(\mathcal{B}_{s}^{\bullet}\right)$ and has $D_{h}$-length at most $A$.

Let $E$ be the event that the above numbered conditions hold, so that $\mathbb{P}[E \mid \mathcal{F}] \geq 1 / 2$.

Let $\phi:[0,1] \rightarrow \mathbb{C}$ be a smooth bump function which supported on a compact subset of $B_{2 \varepsilon}(\pi) \backslash \mathcal{B}_{s}^{\bullet}$ and which is identically equal to 1 on $B_{\varepsilon}(\pi) \backslash B_{\alpha}\left(\mathcal{B}_{s}^{\bullet}\right)$.

Recall [GM20a, Lemma 2.1] that $\mathcal{B}_{s}^{\bullet}$ is a local set for $h$, so under the conditional law of $\left.h\right|_{\mathbb{C} \backslash \mathcal{B}_{s}^{\bullet}}$ given $\mathcal{F}$ is that of a zero-boundary GFF on $\mathbb{C} \backslash \mathcal{B}_{s}^{\bullet}$ plus an $\mathcal{F}$-measurable harmonic function. By a 
standard absolute continuity statement for the GFF, if we set

$$
\widetilde{h}:=h-\frac{1}{\xi} \log \left(\frac{A}{\zeta}\right) \phi,
$$

then the conditional laws of $h$ and $\widetilde{h}$ given $\mathcal{F}$ are mutually absolutely continuous. We also note that by Weyl scaling (Axiom III) and since $\phi$ vanishes on $\mathcal{B}_{s}^{\bullet}$, the definition of $\mathcal{B}_{s}^{\bullet}$ is unaffected by replacing $h$ with $\widetilde{h}$. Since we know that $\mathbb{P}[E \mid \mathcal{F}]>0$, it therefore suffices to show that if $E$ occurs then every $D_{\widetilde{h}}$-geodesic from 0 to a point outside of $B_{\delta}\left(\mathcal{B}_{s}^{\bullet}\right)$ passes through $I$.

Henceforth assume that $E$ occurs. The rest of the argument is deterministic. By Weyl scaling the three conditions in the definition of $E$ lead to the following properties of $D_{\widetilde{h}}$.

1. Since $\phi$ vanishes outside of $B_{2 \varepsilon}(\pi)$, the $D_{\widetilde{h}}$-distance from the $2 \varepsilon$-neighborhood $B_{2 \varepsilon}(\pi)$ to each of $\partial \mathcal{B}_{s}^{\bullet} \backslash I$ and $\partial B_{\delta}\left(\mathcal{B}_{s}^{\bullet}\right)$ is at least $100 \zeta$.

2. Since $\phi$ is non-negative, $D_{\widetilde{h}} \leq D_{h}$ so in particular the $D_{\widetilde{h}^{-}}$-distance from each point of $B_{2 \alpha}\left(\mathcal{B}_{s}^{\bullet}\right)$ to $\mathcal{B}_{s}^{\bullet}$ is at most $\zeta$.

3. Since $\phi \equiv 1$ on $B_{\varepsilon}(\pi) \backslash B_{\alpha}\left(\mathcal{B}_{s}^{\bullet}\right)$, for each $z \in \pi \backslash B_{2 \alpha}\left(\mathcal{B}_{s}^{\bullet}\right)$, there is a path from $z$ to a point of $B_{2 \alpha}\left(\mathcal{B}_{s}^{\bullet}\right)$ which is contained in $B_{\varepsilon}(\pi) \backslash B_{2 \alpha}\left(\mathcal{B}_{s}^{\bullet}\right)$ and has $D_{\widetilde{h}}$-length at most $\zeta$.

Properties 2 and 3 together imply that the $D_{\widetilde{h}}$-distance from each point of $\pi$ to $\mathcal{B}_{s}^{\bullet}$ is at most $2 \zeta$, and hence the $D_{\widetilde{h}}$-distance from each point of $\pi$ to 0 is at most $s+2 \zeta$. Property 1 implies that the $D_{\widetilde{h}}$-distance from each point of $\pi$ to $\mathcal{B}_{s}^{\bullet} \backslash I$ is at least $100 \zeta$. Consequently, every path from 0 to any point of $\pi$ which does not pass through $I$ has $D_{\widetilde{h}^{-}}$-length at least $s+100 \zeta$, so cannot be a $D_{\tilde{h}^{\prime}}$-geodesic. In other words, every $D_{\tilde{h}^{-}}$-geodesic from 0 to any point of $\pi$ passes through $I$. Since $\pi$ disconnects $\mathcal{B}_{s}^{\bullet}$ from $\partial B_{\delta}\left(\mathcal{B}_{s}^{\bullet}\right)$, every $D_{\widetilde{h}}$-geodesic from 0 to any point outside of $B_{\delta}\left(\mathcal{B}_{s}^{\bullet}\right)$ must pass through $\pi$, and hence must also pass through $I$.

Lemma 5.3. Fix $s>0$. Almost surely, the Euclidean dimension of every $D_{h}$-geodesic from 0 to a point of $\partial \mathcal{B}_{s}^{\bullet}$ is equal to $\Delta_{\text {geo }}$.

Proof. Fix $t \in(0, s)$ and $\varepsilon>0$. Let $\mathcal{X}=\mathcal{X}_{t-\varepsilon, t} \subset \partial \mathcal{B}_{t-\varepsilon}^{\bullet}$ be the set of confluence points from Theorem 3.1 so that every leftmost $D_{h}$-geodesic from 0 to a point of $\partial \mathcal{B}_{t}^{\bullet}$ passes through some $x \in \mathcal{X}$. As in Theorem 3.1, for $x \in \mathcal{X}$, let $I_{x} \subset \partial \mathcal{B}_{t}^{\bullet}$ be the set of $y \in \partial \mathcal{B}_{t}^{\bullet}$ such that the leftmost geodesic from 0 to $y$ passes through $x$. Note that $\mathcal{X}$ and the sets $I_{x}$ for $x \in \mathcal{X}$ are $\sigma\left(\mathcal{B}_{t}^{\bullet},\left.h\right|_{\mathcal{B}_{t}^{\bullet}}\right)$-measurable.

By assertion 2 of Theorem 3.1, a.s. there is a unique $D_{h}$-geodesic $P_{x}$ from 0 to each $x \in \mathcal{X}$. We claim that a.s. $\operatorname{dim}_{\mathcal{H}}^{0} P_{x}=\Delta_{\text {geo }}$ for each $x \in \mathcal{X}$ for which $I_{x}$ is not a singleton. Given the claim, we can conclude the proof as follows. By Proposition 3.2, a.s. each $D_{h}$-geodesic from 0 to a point of $\mathbb{C} \backslash \mathcal{B}_{t}^{\bullet}$ passes through some $x \in \mathcal{X}$ for which $I_{x}$ is not a singleton. By the uniqueness of $P_{x}$, each such $D_{h}$-geodesic coincides with $P_{x}$ on the time interval $[0, t-\varepsilon]$. In particular, each $D_{h}$-geodesic $P$ from 0 to a point of $\partial \mathcal{B}_{s}^{\bullet}$ satisfies $\operatorname{dim}_{\mathcal{H}}^{0} P([0, t-\varepsilon])=\Delta_{\text {geo }}$. Sending $t \rightarrow s$ and $\varepsilon \rightarrow 0$ then concludes the proof.

To prove the above claim, let $x_{*} \in \mathcal{X}$ be chosen in a $\sigma\left(\mathcal{B}_{t}^{\bullet},\left.h\right|_{\mathcal{B}_{t}^{\bullet}}\right)$-measurable manner and assume that $I_{x_{*}}$ is not a singleton. By condition 3 of Theorem 3.1, $I_{x_{*}}$ is a non-trivial connected arc of $\partial \mathcal{B}_{t}^{\bullet}$. By Lemma 5.2, a.s. it holds with positive conditional probability given $\sigma\left(\mathcal{B}_{t}^{\bullet},\left.h\right|_{\mathcal{B}_{t}^{\bullet}}\right)$ that the

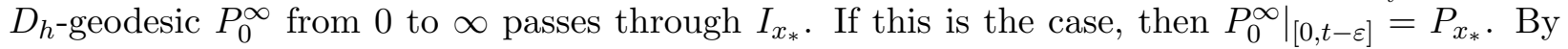
Proposition 5.1, this implies that with positive conditional probability given $\sigma\left(\mathcal{B}_{t}^{*},\left.h\right|_{\mathcal{B}_{t}^{*}}\right)$ we have $\operatorname{dim}_{\mathcal{H}}^{0} P_{x_{*}}=\Delta_{\text {geo }}$. Since $P_{x_{*}}$ is $\sigma\left(\mathcal{B}_{t}^{\bullet},\left.h\right|_{\mathcal{B}_{t}^{\bullet}}\right)$-measurable, in fact a.s. $\operatorname{dim}_{\mathcal{H}}^{0} P_{x_{*}}=\Delta_{\text {geo. }}$. Applying the same argument to each possible choice of $x_{*} \in \mathcal{X}$ gives our claim. 
Lemma 5.4. Fix $s>0$ and $z \in \mathbb{C}$. On the event $\left\{s<D_{h}(0, z)\right\}$, a.s. the Euclidean dimension of every $D_{h}$-geodesic from 0 to a point of $\partial \mathcal{B}_{s}^{z, \bullet}$ is equal to $\Delta_{\text {geo }}$.

Proof. This follows from exactly the same conformal invariance / absolute continuity argument used in the proof of Proposition 3.6.

Proof of Theorem 1.8. By Lemma 5.4, it is a.s. the case that for each $q \in \mathbb{Q}^{2}$ and each rational $s<D_{h}(0, q)$, the Euclidean dimension of every $D_{h}$-geodesic from 0 to a point of $\partial \mathcal{B}_{s}^{q, \bullet}$ is equal to $\Delta_{\text {geo }}$. Henceforth assume that this is the case.

Now let $P$ be a $D_{h}$-geodesic from 0 to a point $z \in \mathbb{C} \backslash\{0\}$. Choose a rational time $s \in\left(0, D_{h}(0, z)\right)$ and let $q \in \mathbb{Q}^{2}$ be a point which lies in the same connected component of $\mathbb{C} \backslash \mathcal{B}_{s}^{\bullet}$ as $z$. Then $\left.P\right|_{[0, s]}$ is a $D_{h}$-geodesic from 0 to a point of $\partial \mathcal{B}_{s}^{q, \bullet}$, so $\operatorname{dim}_{\mathcal{H}}^{0}\left(\left.P\right|_{[0, s]}\right)=\Delta_{\text {geo }}$. Sending $s \rightarrow D_{h}(0, z)$ and using the countable stability of Euclidean dimension then shows that $\operatorname{dim}_{\mathcal{H}}^{0} P=\Delta_{\text {geo }}$.

\section{LQG metric ball boundaries}

In the previous section, we proved a zero-one law for LQG geodesics. For LQG metric ball boundaries, we can prove an even stronger result: Theorem 1.7 identifies the explicit a.s. $\gamma$-quantum and Euclidean dimensions of an LQG metric ball stopped at the first time it hits a specified point. The reason we can obtain this stronger result is that we can apply the earlier work of [Gwy20a] that identified the essential supremum of the $\gamma$-quantum and Euclidean dimensions of LQG metric ball boundaries. We will also apply a more general result from [Gwy20a] that gives upper bounds for the dimensions of certain subsets of LQG metric ball boundaries.

In Section 6.1, we state this generalized upper bound from [Gwy20a], and we prove a couple of technical lemmas that we need to apply this result to our setting. In Section 6.2 we derive a zero-one law for metric ball boundaries, and we use it and the generalized upper bound to prove Theorem 1.7. Finally, in Section 6.3, we will apply the generalized upper bound to analyze the exterior boundaries of LQG metric balls, which we defined in Definition 1.13.

\subsection{A generalized upper bound}

The proofs in Sections 6.2 and 6.3 will use the generalized upper bound theorem [Gwy20a, Theorem 2.9], which gives upper bounds for the $\gamma$-quantum and Euclidean dimensions of a large class of subsets of LQG metric ball boundaries. We restate this theorem here. We have chosen to state the theorem in slightly less than its full generality only to avoid introducing extra notation that we do not need for our applications.

Theorem 6.1 (Generalized upper bound). Suppose that we are given events $\left\{F_{\varepsilon}(z): \varepsilon>0, z \in \mathbb{C}\right\}$ and $q>0$ with the following properties. For any $\alpha \in[-2,2]$, any $\zeta \in(0,1)$, any bounded open set $V \subset \mathbb{C}$ with $\bar{V} \subset \mathbb{C} \backslash\{0\}$, the following is true.

1. For each $z \in V$,

$$
\mathbb{P}\left[F_{\varepsilon}(z) \cap\left\{\sup _{u, v \in B_{\varepsilon}(z)} D_{h}(u, v) \in\left[\varepsilon^{\xi(Q-\alpha)+\zeta}, \varepsilon^{\xi(Q-\alpha)-\zeta}\right]\right\}\right] \leq \varepsilon^{\alpha^{2} / 2+q+o_{\zeta}(1)+o_{\varepsilon}(1)},
$$

where the rate of the $o_{\zeta}(1)$ depends only on $\alpha, \gamma$ and the rate of the $o_{\varepsilon}(1)$ depends only on $V, \alpha, \zeta, \gamma$ (not on the particular choice of $z$ ). ${ }^{3}$

\footnotetext{
${ }^{3}$ Here and in what follows, for two functions $f, g$ of a positive real number $x$ we write $f(x)=o_{x}(g(x))$ (resp. $\left.f(x)=O_{x}(g(x))\right)$ if $f(x) / g(x)$ goes to zero (resp. remains bounded) as $x \rightarrow 0$. The dependencies of the rate of convergence will always be specified unless they are clear from the context.
} 
2. There exists an open set $U \subset \mathbb{C}$ which contains zero and lies at positive distance from $V$ such that for each small enough $\varepsilon>0$ (depending on $V$ ), each of the events $F_{\varepsilon}(z)$ for $z \in V$ is a.s. determined by $\left.h\right|_{\mathbb{C} \backslash U}$.

For $s>0$, let $\mathcal{Y}_{s}$ be the set of $z \in \partial \mathcal{B}_{s}$ such that

$$
\bigcup_{\varepsilon>0} \bigcap_{r \in(0, \varepsilon) \cap \mathbb{Q}} \bigcap_{w \in B_{r}(z) \cap \mathbb{Q}^{2}} F_{r}(w)
$$

occurs, i.e., for each small enough rational $r>0$, the event $F_{r}(w)$ occurs for every $w \in B_{r}(z) \cap \mathbb{Q}^{2}$ (we consider rational values of $r$ and $w$ to avoid measurability issues). Then, almost surely,

$$
\operatorname{dim}_{\mathcal{H}}^{0} Y_{s} \leq \max \left\{0,2-\xi Q+\xi^{2} / 2-q\right\}
$$

and

$$
\operatorname{dim}_{\mathcal{H}}^{\gamma} Y_{s} \leq \max \left\{0, \sup _{\alpha \in[-2,2]} \frac{2-\alpha^{2} / 2-q}{\xi(Q-\alpha)}-1\right\}
$$

We will apply Theorem 6.1 for a particular type of events $F_{\varepsilon}(z)$. Roughly speaking, we define some "good" event $G_{r}(z)$ that depends locally on $h$, viewed modulo additive constant, and has uniformly positive probability across scales. We let $F_{\varepsilon}(z)$ be the "very bad" event that none of the events $G_{r}(z)$ occur for a particular range of $r$ values (depending on $\varepsilon$ ). The following lemma asserts that the very bad events $F_{\varepsilon}(z)$ satisfy the conditions of the generalized upper bound. If we think of "very bad points" $z$ as points for which $F_{\varepsilon}(z)$ occurs, then the generalized upper bound a.s. bounds the dimension of points "surrounded" by very bad points on some sufficiently small scale.

Lemma 6.2. Suppose that $G_{r}(z)$ is an event defined for each $z \in \mathbb{C}$ and $r>0$ with the following two properties:

- The probability of $G_{r}(z)$ is positive and does not depend on $r$ or $z$.

- There exists $b>a>0$ such that for each $z, r$, the event $G_{r}(z)$ is a.s. determined by $\left.h\right|_{B_{b r}(z) \backslash B_{a r}(z)}$, viewed modulo additive constant.

Then the event

$$
F_{\varepsilon}(z):=\bigcap_{r \in\left[\varepsilon^{1 / 2} / a, 2 \varepsilon^{1 / 4} / b\right] \cap \mathbb{Q}}\left[G_{r}(z)\right]^{c}
$$

satisfies the conditions of Theorem 6.1 for some $q>0$.

To prove Lemma 6.2 , we first check that $F_{\varepsilon}(z)$ is sufficiently local and that its probability (not intersected with any other event) decays sufficiently fast as $\varepsilon \rightarrow 0$ :

Lemma 6.3. With $F_{\varepsilon}(z)$ defined just above, $F_{\varepsilon}(z)$ is a.s. determined by $\left.h\right|_{B_{2 \varepsilon^{1 / 4}}(z) \backslash B_{\varepsilon^{1 / 2}}(z)}$ viewed modulo additive constant. Furthermore, there is an exponent $q=q(\gamma)>0$ such that $\mathbb{P}\left[F_{\varepsilon}(z)\right]=$ $O_{\varepsilon}\left(\varepsilon^{q}\right)$ uniformly over all $z \in \mathbb{C}$.

Proof. From the locality of $G_{r}(z)$ and the definition of $F_{\varepsilon}(z)$, it is immediate that $F_{\varepsilon}(z)$ is a.s. determined by $\left.h\right|_{B_{2 \varepsilon^{1 / 4}}(z) \backslash B_{\varepsilon^{1 / 2}}(z)}$ viewed modulo additive constant.

To prove the second part of the lemma, let $p=p(\gamma)>0$ be a constant such that $\mathbb{P}\left[G_{r}(z)\right] \geq p$ for each $z \in \mathbb{C}$ and $r>0$. We can now use a general independence lemma for the restrictions of the GFF in disjoint concentric annuli [GM20b, Lemma 3.1] to find that there is a $q=q(\gamma)>0$ such that $\mathbb{P}\left[F_{\varepsilon}(z)\right]=O_{\varepsilon}\left(\varepsilon^{q}\right)$. 
We now show that, for any events $F_{\varepsilon}(z)$ with the two properties we proved in Lemma $6.3, F_{\varepsilon}(z)$ satisfies the upper bound (6.1) for the probability of $F_{\varepsilon}(z)$ intersected with the event that the $D_{h}$-diameter of $B_{\varepsilon}(z)$ lies in a certain interval.

Lemma 6.4. Suppose $q>0$ and we are given events $F_{\varepsilon}(z)$ for $\varepsilon>0$ and $z \in \mathbb{C}$ such that $F_{\varepsilon}(z)$ is a.s. determined by $\left.h\right|_{\mathbb{C} \backslash B_{\varepsilon 1 / 2}(z)}$, viewed modulo additive constant, and $\mathbb{P}\left[F_{\varepsilon}(z)\right]=O_{\varepsilon}\left(\varepsilon^{q}\right)$ as $\varepsilon \rightarrow 0$, uniformly over all $z \in \mathbb{C}$. Also let $\alpha \in[-2,2]$ and $\zeta \in(0,1)$ and let $V \subset \mathbb{C}$ be bounded open set with $\bar{V} \subset \mathbb{C} \backslash\{0\}$. Then for each $z \in V$,

$$
\mathbb{P}\left[F_{\varepsilon}(z) \cap\left\{\sup _{u, v \in B_{\varepsilon}(z)} D_{h}(u, v) \in\left[\varepsilon^{\xi(Q-\alpha)+\zeta}, \varepsilon^{\xi(Q-\alpha)-\zeta}\right]\right\}\right] \leq \varepsilon^{\alpha^{2} / 2+q+o_{\zeta}(1)+o_{\varepsilon}(1)}
$$

where the rate of convergence of the $o_{\zeta}(1)$ depends only on $\alpha, \gamma$ and the rate of convergence of the $o_{\varepsilon}(1)$ depends only on $V, \alpha, \zeta, \gamma$.

Proof. To lighten notation, let

$$
H_{\varepsilon}(z):=\left\{\sup _{u, v \in B_{\varepsilon}(z)} D_{h}(u, v) \in\left[\varepsilon^{\xi(Q-\alpha)+\zeta}, \varepsilon^{\xi(Q-\alpha)-\zeta}\right]\right\} .
$$

By Lemma 6.3 and a basic estimate for $D_{h}$-diameters (see, e.g., [Gwy20a, Lemma 2.3]), for each $z \in V$ we have

$$
\mathbb{P}\left[F_{\varepsilon}(z)\right]=O_{\varepsilon}\left(\varepsilon^{q}\right) \quad \text { and } \quad \mathbb{P}\left[H_{\varepsilon}(z)\right] \leq \varepsilon^{\alpha^{2} / 2+o_{\zeta}(1)+o_{\varepsilon}(1)} .
$$

The idea of the proof is that $F_{\varepsilon}(z)$ depends only on $\left.h\right|_{\mathbb{C} \backslash B_{\varepsilon^{1 / 2}(z)}}$ viewed modulo additive constant, whereas $H_{\varepsilon}(z)$ is (almost) determined by $\left.h\right|_{3 \varepsilon}(z)$, so $F_{\varepsilon}(z)$ and $H_{\varepsilon}(z)$ are approximately independent. However, $H_{\varepsilon}(z)$ is not exactly determined by $\left.h\right|_{B_{3 \varepsilon}(z)}$ since in $D_{h}$-geodesics paths between points of $B_{\varepsilon}(z)$ could get very far from $B_{\varepsilon}(z)$. So, to make the above idea precise we need to introduce a localized version of $H_{\varepsilon}(z)$.

Step 1: localizing $H_{\varepsilon}(z)$. Let $\widetilde{H}_{\varepsilon}(z)$ be the event that the following is true.

1. There is a path in $B_{2 \varepsilon}(z) \backslash B_{\varepsilon}(z)$ which disconnects the inner and outer boundaries of $B_{2 \varepsilon}(z) \backslash B_{\varepsilon}(z)$ whose length is at most $\varepsilon^{-\zeta} D_{h}\left(\partial B_{2 \varepsilon}(z), \partial B_{3 \varepsilon}(z)\right)$.

2. We have $\sup _{u, v \in B_{\varepsilon}(z)} D_{h}\left(u, v ; B_{3 \varepsilon}(z)\right) \in\left[\varepsilon^{\xi(Q-\alpha)+\zeta}, \varepsilon^{\xi(Q-\alpha)-2 \zeta}\right]$.

By the locality of the metric (Axiom II), $\widetilde{H}_{\varepsilon}(z)$ is a.s. determined by $\left.h\right|_{B_{3 \varepsilon}(z)}$. Furthermore, the proof of [Gwy20a, Lemma 2.3] shows that

$$
\mathbb{P}\left[\widetilde{H}_{\varepsilon}(z)\right] \leq \varepsilon^{\alpha^{2} / 2+o_{\zeta}(1)+o_{\varepsilon}(1)}, \quad \forall z \in V .
$$

We now argue that

$$
\mathbb{P}\left[H_{\varepsilon}(z) \backslash \widetilde{H}_{\varepsilon}(z)\right]=O_{\varepsilon}\left(\varepsilon^{N}\right), \quad \forall N \in \mathbb{N},
$$

uniformly over all $z \in \mathbb{C}$. We first claim that the probability that condition 1 in the definition of $\widetilde{H}_{\varepsilon}(z)$ fails to occur decays faster than any positive power of $\varepsilon$ as $\varepsilon \rightarrow 0$. Indeed, this follows from $\left[\mathrm{DFG}^{+}\right.$20, Proposition 3.1], applied to compare each of the distance "around" $B_{2 \varepsilon}(z) \backslash B_{\varepsilon}(z)$ and the distance "across" $B_{3 \varepsilon}(z) \backslash B_{2 \varepsilon}(z)$ to the quantity $(2 \varepsilon)^{\xi Q} e^{\xi h_{2 \varepsilon}(z)}$.

We will now conclude the proof of (6.8) by showing that if both $H_{\varepsilon}(z)$ and condition 1 in the definition of $\widetilde{H}_{\varepsilon}(z)$ occur, then $\widetilde{H}_{\varepsilon}(z)$ occurs. Indeed, if $H_{\varepsilon}(z)$ occurs then $\sup _{u, v \in B_{\varepsilon}(z)} D_{h}\left(u, v ; B_{3 \varepsilon}(z)\right) \geq$ 
$\sup _{u, v \in B_{\varepsilon}(z)} D_{h}(u, v) \geq \varepsilon^{\xi(Q-\alpha)+\zeta}$. To get the bound in the other direction, suppose $u, v \in B_{\varepsilon}(z)$ and let $P$ be a $D_{h}$-geodesic from $u$ to $v$. If $P$ is contained in $B_{3 \varepsilon}(z)$ then $D_{h}(u, v)=D_{h}\left(u, v ; B_{3 \varepsilon}(z)\right)$. Otherwise, we can replace a segment of $P$ by a segment of the path in $B_{2 \varepsilon}(z) \backslash B_{\varepsilon}(z)$ from condition 1 in the definition of $\widetilde{H}_{\varepsilon}(z)$ to get a new path from $u$ to $v$ which stays in $B_{3 \varepsilon}(z)$ and whose $D_{h}$-length is at most $\varepsilon^{-\zeta} D_{h}(u, v)$. Therefore, $D_{h}\left(u, v ; B_{3 \varepsilon}(z)\right) \leq \varepsilon^{-\zeta} D_{h}(u, v)$. By the definition of $H_{\varepsilon}(z)$, we infer that $\widetilde{H}_{\varepsilon}(z)$ occurs, and hence $(6.8)$ holds.

Step 2: near-independence of $F_{\varepsilon}(z)$ and $H_{\varepsilon}(z)$. Let $\bar{h}$ be an independent copy of $h$. By a basic estimate for the GFF (see, e.g., [GMS19, Lemma A.5] applied with $\delta=3 \varepsilon^{1 / 2}$ combined with the fact that $h(3 \varepsilon / \cdot) \stackrel{d}{=} h$ modulo additive constant), we get that for some universal constant $a \in(0,1)$, the following is true. The conditional law of $\left.h\right|_{\mathbb{C} \backslash B_{\varepsilon^{1 / 2}}(z)}$, viewed modulo additive constant, given $\left.h\right|_{B_{3 \varepsilon}(z)}$ is mutually absolutely continuous w.r.t. the unconditional law of $\left.\bar{h}\right|_{\mathbb{C} \backslash B_{\varepsilon^{1 / 2}}(z)}$, viewed modulo additive constant. Furthermore, if $M=M\left(\left.h\right|_{B_{3 \varepsilon}(z)},\left.\bar{h}\right|_{\mathbb{C} \backslash B_{\varepsilon^{1 / 2}}(z)}\right)$ is the Radon-Nikodym derivative of the former law w.r.t. the latter law, then for $\varepsilon \in(0, a]$ the $a / \varepsilon^{1 / 2}$-th moments of $M$ and its reciprocal are each bounded above by a universal constant.

Let $\bar{F}_{\varepsilon}(z)$ be defined in the same manner as $F_{\varepsilon}(z)$ but with $\bar{h}$ in place of $h$. Since $\widetilde{H}_{\varepsilon}(z) \in$ $\sigma\left(\left.h\right|_{B_{3 \varepsilon}(z)}\right)$ and $F_{\varepsilon}(z)$ is a.s. determined by $\left.h\right|_{\mathbb{C} \backslash B_{\varepsilon^{1 / 2}}(z)}$, viewed modulo additive constant,

$$
\mathbb{P}\left[F_{\varepsilon}(z) \cap \widetilde{H}_{\varepsilon}(z)\right]=\mathbb{E}\left[\mathbb{P}\left[F_{\varepsilon}(z)|h|_{B_{3 \varepsilon}(z)}\right] \mathbb{1}_{\widetilde{H}_{\varepsilon}(z)}\right]=\mathbb{E}\left[\mathbb{E}\left[M \mathbb{1}_{\bar{F}_{\varepsilon}(z)}|h|_{B_{3 \varepsilon}(z)}\right] \mathbb{1}_{\widetilde{H}_{\varepsilon}(z)}\right] .
$$

To bound the conditional expectation in (6.9), we use Hölder's inequality (with exponents $a / \varepsilon^{1 / 2}$ and $\left.1 /\left(1-\varepsilon^{1 / 2} / a\right)\right)$ to get

$$
\begin{aligned}
\mathbb{E}\left[M \mathbb{1}_{\bar{F}_{\varepsilon}(z)}|h|_{B_{3 \varepsilon}(z)}\right] & \leq \mathbb{E}\left[M^{a / \varepsilon^{1 / 2}}|h|_{B_{3 \varepsilon}(z)}\right]^{\varepsilon^{1 / 2} / a} \mathbb{P}\left[\bar{F}_{\varepsilon}(z)|h|_{B_{3 \varepsilon}(z)}\right]^{1-\varepsilon^{1 / 2} / a} \\
& =\mathbb{E}\left[M^{a / \varepsilon^{1 / 2}}|h|_{B_{3 \varepsilon}(z)}\right]^{\varepsilon^{1 / 2} / a} \mathbb{P}\left[F_{\varepsilon}(z)\right]^{1-\varepsilon^{1 / 2} / a}
\end{aligned}
$$

where in the last line we used that $\bar{h} \stackrel{d}{=} h$ and $\bar{h}$ is independent from $h$. We now plug (6.10) into (6.9) and apply Hölder's inequality a second time (with the same exponents) to get

$$
\begin{aligned}
\mathbb{P}\left[F_{\varepsilon}(z) \cap \widetilde{H}_{\varepsilon}(z)\right] & \leq \mathbb{P}\left[F_{\varepsilon}(z)\right]^{1-\varepsilon^{1 / 2} / a} \mathbb{E}\left[\mathbb{E}\left[M^{a / \varepsilon^{1 / 2}}|h|_{B_{3 \varepsilon}(z)}\right]^{\varepsilon^{1 / 2} / a} \mathbb{1}_{\widetilde{H}_{\varepsilon}(z)}\right] \\
& \leq \mathbb{P}\left[F_{\varepsilon}(z)\right]^{1-\varepsilon^{1 / 2} / a} \mathbb{E}\left[M^{a / \varepsilon^{1 / 2}}\right]^{\varepsilon^{1 / 2} / a} \mathbb{P}\left[\widetilde{H}_{\varepsilon}(z)\right]^{1-\varepsilon^{1 / 2} / a} \quad \text { (by Hölder) } \\
& \leq \text { const. } \times \mathbb{P}\left[F_{\varepsilon}(z)\right]^{1-\varepsilon^{1 / 2} / a} \mathbb{P}\left[\widetilde{H}_{\varepsilon}(z)\right]^{1-\varepsilon^{1 / 2} / a} \quad \text { (by our estimate for } M \text { ) } .
\end{aligned}
$$

The lemma now follows by using (6.8) to upper-bound $\mathbb{P}\left[F_{\varepsilon}(z) \cap H_{\varepsilon}(z)\right]$ in terms of $\mathbb{P}\left[F_{\varepsilon}(z) \cap \widetilde{H}_{\varepsilon}(z)\right]$, then using (6.7) and the fact that $\mathbb{P}\left[F_{\varepsilon}(z)\right]=O_{\varepsilon}\left(\varepsilon^{q}\right)$ to upper-bound the right side of (6.11).

Proof of Lemma 6.2. The lemma follows from Lemmas 6.3 and 6.4.

\subsection{The Hausdorff dimension of metric ball boundaries}

In this subsection, we prove Theorem 1.7. We begin by proving the following zero-one law for the dimension of metric balls started from $\infty$. 
Proposition 6.5. Define $\mathcal{B}_{0}^{\infty}$ as in Proposition 4.7. There are deterministic constants $\Delta_{\text {ball }}^{0}, \Delta_{\text {ball }}^{\gamma}>$ 0 such that a.s. $\operatorname{dim}_{\mathcal{H}}^{0} \partial \mathcal{B}_{0}^{\infty}=\Delta_{\text {ball }}^{0}$ and $\operatorname{dim}_{\mathcal{H}}^{\gamma} \partial \mathcal{B}_{0}^{\infty}=\Delta_{\text {ball }}^{\gamma}$. Furthermore, for each fixed $z \in \mathbb{C}$ a.s. $\operatorname{dim}_{\mathcal{H}}^{0} \partial \mathcal{B}_{D_{h}(z, w)}\left(w ; D_{h}\right) \geq \Delta_{\text {ball }}^{0}$ and $\operatorname{dim}_{\mathcal{H}}^{\gamma} \partial \mathcal{B}_{D_{h}(z, w)}\left(w ; D_{h}\right) \geq \Delta_{\text {ball }}^{\gamma}$ simultaneously for each $w \in \mathbb{C}$.

Proof. We prove the result for Euclidean dimensions; the proof of the result for $\gamma$-quantum dimensions is essentially the same. The basic strategy of the proof is similar to the proofs of Theorem 1.11 and Proposition 5.1.

Let $c>0$ such that $\mathbb{P}\left[\operatorname{dim}_{\mathcal{H}}^{0} \partial \mathcal{B}_{0}^{\infty} \geq c\right]>0$. We will show that a.s.

$$
\operatorname{dim}_{\mathcal{H}}^{0}\left(\partial \mathcal{B}_{0}^{\infty} \cap B_{r}(0)\right) \geq c, \quad \forall r>0 .
$$

From (6.12), we immediately get that $\operatorname{dim}_{\mathcal{H}}^{0} \partial \mathcal{B}_{0}^{\infty}$ is a.s. equal to a deterministic constant $\Delta_{\text {ball }}^{0}$. Furthermore, by combining (6.12) (applied with $c=\Delta_{\text {ball }}^{0}$ ) with (4.7) of Proposition 4.7, we get that there is a large $R>0$ such that a.s. $\operatorname{dim}_{\mathcal{H}}^{0} \partial \mathcal{B}_{D_{h}(0, w)}\left(w ; D_{h}\right) \geq \Delta_{\text {ball }}^{0}$ for each $w \in \mathbb{C} \backslash B_{R}(0)$. By the scale invariance of the law of $h$, modulo additive constant, and (4.8) we can remove the restriction that $w \in \mathbb{C} \backslash B_{R}(0)$. This gives the second statement of the proposition with $z=0$. The statement for a general $z \in \mathbb{C}$ follows from the translation invariance of the law of $h$ modulo additive constant.

Let us now prove (6.12). By the countable stability of Hausdorff dimension, for each $\delta \in(0,1)$ there exists $r_{0}>0$ and $p \in(0,1)$ such that $\mathbb{P}\left[\operatorname{dim}_{\mathcal{H}}^{0}\left(\partial \mathcal{B}_{0}^{\infty} \cap B_{r_{0}}(0)\right) \geq c-\delta\right] \geq p$. By the scale invariance of the law of $\mathcal{B}_{0}^{\infty}$ (see (4.8)), this implies that in fact

$$
\mathbb{P}\left[\operatorname{dim}_{\mathcal{H}}^{0}\left(\partial \mathcal{B}_{0}^{\infty} \cap B_{r}(0)\right) \geq c-\delta\right] \geq p, \quad \forall r>0 .
$$

We now want to use a tail triviality argument to deduce (6.12) from (6.13). To this end, let $A=A(1-p / 2, \gamma)$ be as in Lemma 4.8 with $1-p / 2$ in place of $p$. For $r>0$, let $E_{r}=E_{r}(0)$ and $Z_{r}=Z_{r}(0)$ be the point and event from that lemma, so that $E_{r} \in \sigma\left(\left.h\right|_{B_{A^{2} r}(0)}\right)$ and $\mathbb{P}\left[E_{r}\right] \geq 1-p / 2$. Let

$$
G_{r}:=E_{r} \cap\left\{\operatorname{dim}_{\mathcal{H}}^{0}\left(\partial \mathcal{B}_{0}^{\infty} \cap B_{A^{2} r}(0)\right) \geq c-\delta\right\} .
$$

By (6.13), we have $\mathbb{P}\left[G_{r}\right] \geq p / 2$.

We now argue that $G_{r} \in \sigma\left(\left.h\right|_{B_{A^{2} r}(0)}\right)$. Indeed, we recall from Lemma 4.8 that on $E_{r}$, every $D_{h}$-geodesic from a point of $B_{r}(0)$ to a point of $\mathbb{C} \backslash B_{A r}(0)$ passes through $Z_{r}$. Hence the proof of (4.7) of Proposition 4.7 shows that if $E_{r}$ occurs, then

$$
\mathcal{B}_{0}^{\infty} \cap B_{r}(0)=\mathcal{B}_{D_{h}\left(0, Z_{r}\right)}\left(Z_{r} ; D_{h}\right) \cap B_{r}(0)
$$

On the other hand, Lemma 4.8 shows that on $E_{r}$, every $D_{h}$-geodesic from $Z_{r}$ to a point of $B_{r}(0)$ stays in $B_{A^{2} r}(0)$, which means that $D_{h}\left(0, Z_{r}\right)=D_{h}\left(0, Z_{r} ; B_{A^{2} r}(0)\right)$ and

$$
\mathcal{B}_{D_{h}\left(0, Z_{r}\right)}\left(Z_{r} ; D_{h}\right) \cap B_{r}(0)=\mathcal{B}_{D_{h}\left(0, Z_{r} ; B_{A^{2} r}(0)\right)}\left(Z_{r} ; D_{h}\left(\cdot, \cdot ; B_{A^{2} r}(0)\right)\right) \cap B_{r}(0) .
$$

The right side of (6.16) is $\sigma\left(\left.h\right|_{B_{A^{2} r}(0)}\right)$-measurable due to Axiom II. By (6.15) and (6.16), we therefore obtain that $G_{r} \in \sigma\left(\left.h\right|_{B_{A^{2} r}(0)}\right)$, as desired.

Since $\mathbb{P}\left[G_{r}\right] \geq p / 2$, it holds with positive probability that there is a sequence $r_{k} \rightarrow 0$ for which $G_{r_{k}}$ occurs. Since $G_{r} \in \sigma\left(\left.h\right|_{B_{A^{2} r}(0)}\right)$ and $\bigcap_{r>0} \sigma\left(\left.h\right|_{B_{A^{2} r}(0)}\right)$ is trivial, the probability that such a sequence exists is equal to zero or one, so such a sequence must exist a.s. Recalling the definition (6.14) of $G_{r}$, we now obtain (6.12). 
We devote the remainder of this subsection to proving Theorem 1.7 from Proposition 6.5. As in the above proof of Proposition 6.5, we will prove just the result for Euclidean dimension, since the proof for $\gamma$-quantum dimension is essentially the same.

To deduce Theorem 1.7 from Proposition 6.5, it suffices to show that $\Delta_{\text {ball }}^{0} \geq 2-\xi Q+\xi^{2} / 2$ and a.s. $\operatorname{dim}_{\mathcal{H}}^{0} \partial \mathcal{B}_{D_{h}(0, z)} \leq 2-\xi Q+\xi^{2} / 2$. We prove the second inequality in Lemma 6.10 below. First we turn to the first inequality, which we state as a proposition.

Proposition 6.6. $\Delta_{\text {ball }}^{0} \geq 2-\xi Q+\xi^{2} / 2$.

We will extract Proposition 6.6 from [Gwy20a, Theorem 1.1], which says the following. Suppose we fix $t>0$ and consider the ball $\mathcal{B}_{t}=\mathcal{B}_{t}\left(0 ; D_{h}\right)$. Then the essential supremum of the law of the random variable $\operatorname{dim}_{\mathcal{H}}^{0}\left(\partial \mathcal{B}_{t}\right)$ is equal to $2-\xi Q+\xi^{2} / 2$.

Roughly speaking, we will deduce Proposition 6.6 from this statement as follows. For a "typical" time $t>0$ and a "typical" point $z \in \partial \mathcal{B}_{t}$, we expect that near $z, \partial \mathcal{B}_{t}$ locally looks like a segment of the boundary of the ball $\mathcal{B}_{0}^{\infty}$ started from $\infty$ and stopped upon hitting 0 , as constructed in Proposition 4.7. In particular, for such a point $z$ and a small enough $\varepsilon>0$ it should be that $\operatorname{dim}_{\mathcal{H}}^{0}\left(B_{\varepsilon}(z) \cap \partial \mathcal{B}_{t}\right) \leq \Delta_{\text {ball }}^{0}$. Say that a point which satisfies this condition for some $\varepsilon>0$ is "normal".

Using Theorem 6.1, we can show that, for any $t>0$, the Hausdorff dimension of the complementary set of "abnormal" points $z \in \partial \mathcal{B}_{t}$ is a.s. at most $2-\xi Q-\xi^{2} / 2-q$ for some $q=q(\gamma)>0$. On the other hand, for Lebesgue-a.e. $t$, the set of "normal" points in $\partial \mathcal{B}_{t}$ has Hausdorff dimension at most $\Delta_{\text {ball }}^{0}$. From this, we deduce that, for such a choice of $t, \operatorname{dim}_{\mathcal{H}}^{0}\left(\partial \mathcal{B}_{t}\right) \leq \max \left\{2-\xi Q+\xi^{2} / 2-q, \Delta_{\text {ball }}^{0}\right\}$ almost surely. But, [Gwy20a, Theorem 1.1] tells us that the essential supremum of the law of the random variable $\operatorname{dim}_{\mathcal{H}}^{0}\left(\partial \mathcal{B}_{t}\right)$ is equal to $2-\xi Q+\xi^{2} / 2$. Hence we must have $\Delta_{\text {ball }}^{0} \geq 2-\xi Q+\xi^{2} / 2$.

To implement this strategy, we will apply Theorem 6.1 via Lemma 6.2. Let $A=A(1 / 2, \gamma)>0$ be as in the statement of Lemma 4.8 with $p=1 / 2$. We define the "bad" events $F_{\varepsilon}(z)$ of Theorem 6.1 as in Lemma 6.2, with $a=1 / A$ and $b=A^{2}$ and the "good" events $G_{r}(z)$ taken to be the confluence events $E_{r}(z)$ of Lemma 4.8 with $p=1 / 2$. We also let $\mathcal{Y}_{t}$ be the set of "bad" points as in Theorem 6.1 with this choice of $F_{\varepsilon}(z)$.

We will motivate the choice of events $G_{r}(z)$ in a moment. First, with $\mathcal{Y}_{t}$ as in Theorem 6.1, we obtain the following upper bound on the Hausdorff dimension of $\mathcal{Y}_{t}$.

Lemma 6.7. There exists $q=q(\gamma)>0$ such that a.s. $\operatorname{dim}_{\mathcal{H}}^{0} \mathcal{Y}_{t} \leq 2-\xi Q+\xi^{2} / 2-q$.

Proof. The result follows from applying Theorem 6.1 via Lemma 6.2; we just need to check that, with our above definitions, the events $G_{r}(z)$ satisfy the two conditions of Lemma 6.2. Indeed, Lemma 4.8 implies that the event $G_{r}(z)$ is a.s. determined by $\left.h\right|_{B_{A^{2} r}(z) \backslash B_{r / A}(z)}$ viewed modulo additive constant, and that $\mathbb{P}\left[G_{r}(z)\right] \geq 1 / 2$ for each $z \in \mathbb{C}$ and $r>0$.

The remaining ingredient we need to prove Proposition 6.6 is to show that $\partial \mathcal{B}_{t} \backslash \mathcal{Y}_{t}$ has Hausdorff dimension at most $\Delta_{\text {ball }}^{0}$ almost surely. This is where the particular definition of the "good" events $G_{r}(z)$-as the confluence events $E_{r}(z)$ of Lemma 4.8-plays a crucial role. Specifically, we will use the following two properties that hold on the event $G_{r}(z)$ by Lemma 4.8:

(a) Each $D_{h}$-geodesic from a point of $B_{r}(z)$ to a point of $\mathbb{C} \backslash B_{A r}(z)$ passes through the single (random) point $Z_{r}(z) \in B_{A r}(z) \backslash B_{r}(z)$.

(b) Each $D_{h}$-geodesic between points of $\mathbb{C} \backslash B_{r}(z)$ is contained in $\mathbb{C} \backslash \overline{B_{r / A}(z)}$. More strongly, there is a path $\pi \subset B_{r}(z) \backslash \overline{B_{r / A}(z)}$ such that

$$
\left(D_{h} \text {-length of } \pi\right)<D_{h}\left(\pi, B_{r / A}(z)\right) .
$$


Here as above, $A>0$ is as in Lemma 4.8 with $p=1 / 2$.

We first prove a lemma that states that, if two "good" events $G_{r}(z)$ and $G_{r^{\prime}}\left(z^{\prime}\right)$ corresponding to nested annuli occur, then we can compare the ball started from infinity and run until it hits $z$ (see Proposition 4.7) to the ball centered at a point in $\mathbb{C}$ and run until a specified time. This comparison is useful because we already know from Proposition 6.5 that the boundary of the ball started from infinity has dimension $\Delta_{\text {ball }}^{0}$ almost surely.

Lemma 6.8. If $z, z^{\prime} \in \mathbb{C}$ and $r^{\prime}>r>0$ are such that $B_{r}(z) \subset B_{r^{\prime}}\left(z^{\prime}\right), B_{r / A}(z) \subset B_{r^{\prime} / A}\left(z^{\prime}\right)$, and $0 \notin B_{A r^{\prime}}\left(z^{\prime}\right)$, then a.s. for Lebesgue-a.e. $t>D_{h}\left(0, B_{r / A}(z)\right)$, the inequality

$$
\operatorname{dim}_{\mathcal{H}}^{0}\left(\partial \mathcal{B}_{t} \cap\left[B_{r^{\prime}}\left(z^{\prime}\right) \backslash B_{r}(z)\right]\right) \leq \Delta_{\text {ball }}^{0}
$$

holds on the event $G_{r}(z) \cap G_{r^{\prime}}\left(z^{\prime}\right)$.

Before presenting the proof of the lemma, we sketch the main steps of the argument. Due to the definition of the metric ball $\mathcal{B}_{z}^{\infty}$ started from $\infty$ and stopped upon hitting $z$, property (a) of $G_{r^{\prime}}\left(z^{\prime}\right)$ directly allows us to show that on $G_{r^{\prime}}\left(z^{\prime}\right)$, we have $\mathcal{B}_{z}^{\infty} \cap B_{r}(z)=\mathcal{B}_{D_{h}(0, z)}\left(0 ; D_{h}\right) \cap B_{r}(z)$. The trickier part of the argument is applying property (b) of $G_{r}(z)$ to compare the LQG balls of radii $D_{h}(0, z)$ and $t$ centered at 0 . To do this, we start the proof by replacing our field $h$ with a field $h^{*}$ which is equal in distribution to $h$ modulo additive constant, defined so that $h^{*}-h$ is a random multiple of a bump function which is supported on $B_{r / A}(z)$. Since $h^{*}=h$ outside of $B_{r / A}(z)$, property (b) of $G_{r}(z)$ says that geodesics between points outside the larger ball $B_{r}(z)$ are the same for both metrics $D_{h}$ and $D_{h^{*}}$. On the other hand, the conditional law given $h$ of the random variable $D_{h^{*}}(0, z)$ is absolutely continuous with respect to Lebesgue measure on the set of times $t>D_{h}\left(0, B_{r / A}(z)\right)$. So, we can translate an almost sure statement about a $D_{h}$-ball with

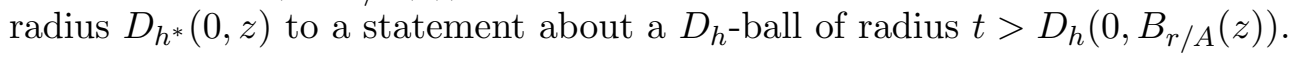

Proof of Lemma 6.8. Let $\phi: \mathbb{C} \rightarrow[0, \infty)$ be a smooth bump function which is positive on $B_{r / A}(z)$, is zero outside $B_{r / A}(z)$, and is normalized so that the Dirichlet energy of $\phi$ satisfies $(\phi, \phi)_{\nabla}=1$. Let $Z$ be a standard Gaussian random variable which is independent from $h$. Consider the field

$$
h^{*}:=h-(h, \phi)_{\nabla} \phi+Z \phi
$$

where $(h, \phi)_{\nabla}$ is the Dirichlet inner product. By the definition of the whole-plane GFF as a sum of i.i.d. standard Gaussians times the elements of an orthonormal basis for the Dirichlet inner product, $h^{*}$ and $h$ are equal in distribution modulo additive constant.

Let $\mathcal{B}_{z}^{\infty, *}$ be defined in the same manner as the ball $\mathcal{B}_{z}^{\infty}$ of Proposition 4.7 but with $h^{*}$ in place of $h$. Since $B_{z}^{\infty, *}$ is determined by $h^{*}$ viewed modulo additive constant, we can apply Proposition 6.5 with $h^{*}$ in place of $h$ to get that a.s. $\operatorname{dim}_{\mathcal{H}}^{0} \partial \mathcal{B}_{z}^{\infty, *}=\Delta_{\text {ball }}^{0}$. We convert this to a statement about the dimension of $\mathcal{B}_{t}\left(0 ; D_{h}\right) \cap B_{r}(z)$ in two stages.

1. Since $B_{r / A}(z) \subset B_{r^{\prime} / A}\left(z^{\prime}\right)$, each of $G_{r}(z)$ and $G_{r^{\prime}}\left(z^{\prime}\right)$ is determined by $\left.h\right|_{\mathbb{C} \backslash B_{r / A}(z)}$, viewed modulo additive constant. So, the definitions of these events are unaffected by replacing $h$ by $h^{*}$. By Property (a) for $G_{r^{\prime}}\left(z^{\prime}\right)$, on $G_{r^{\prime}}\left(z^{\prime}\right)$ every $D_{h^{*} \text {-geodesic from a point outside }}$ $B_{A r^{\prime}}\left(z^{\prime}\right)$ to a point inside $B_{r^{\prime}}\left(z^{\prime}\right)$ passes through the single point $Z_{r^{\prime}}\left(z^{\prime}\right)$. This implies that $\partial \mathcal{B}_{D_{h^{*}}(0, z)}\left(0 ; D_{h^{*}}\right) \cap B_{r^{\prime}}\left(z^{\prime}\right)=\partial \mathcal{B}_{z}^{\infty, *} \cap B_{r^{\prime}}\left(z^{\prime}\right)$.

2. By Property (b) of $G_{r}(z)$, on $G_{r}(z)$ every $D_{h}$-geodesic between two points outside of $B_{r}(z)$ is contained in $\mathbb{C} \backslash B_{r / A}(z)$. Since $h^{*}=h$ outside of $B_{r / A}(z)$, we can use the stronger 

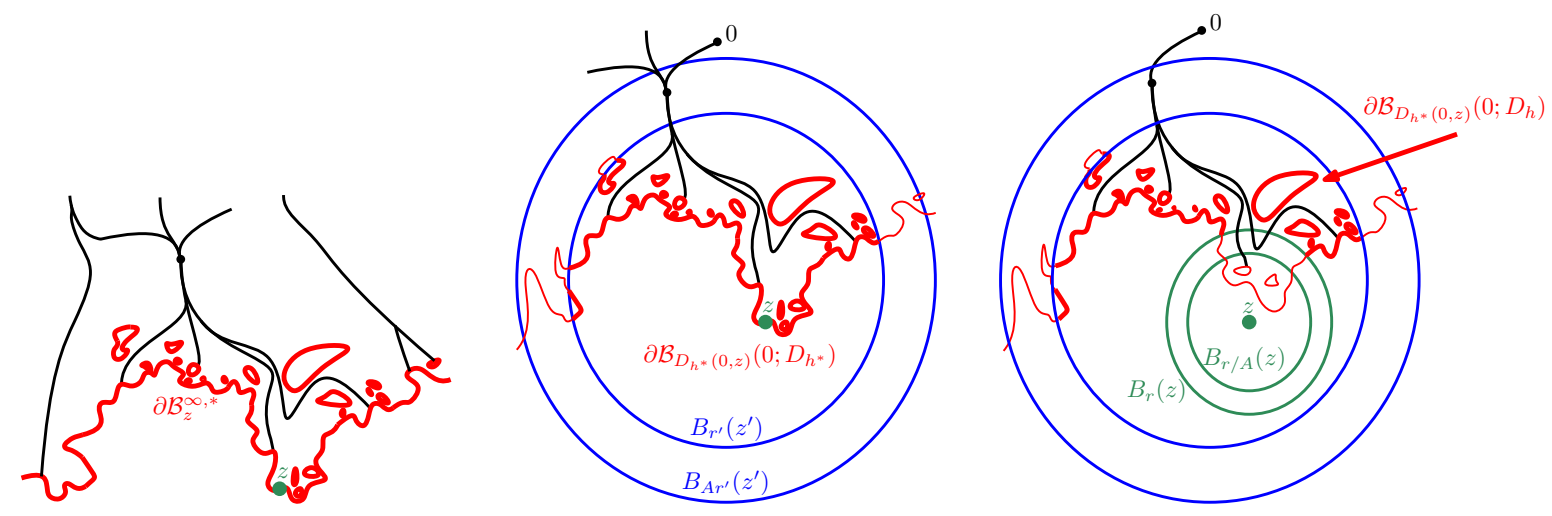

Figure 8: An illustration of the proof of Lemma 6.8. In each figure, the bold part of the red set has Euclidean dimension at most $\Delta_{\text {ball }}^{0}$ almost surely on the event we are considering. Left: We start with the $D_{h^{*}}$-metric ball $\mathcal{B}_{z}^{\infty, *}$ started from $\infty$ and run until hitting $z$; the red set represents its boundary, and the black curves are geodesic rays. Middle: The red set now represents $\partial \mathcal{B}_{D_{h^{*}}(0, z)}\left(0 ; D_{h^{*}}\right)$. By property (a) of $G_{r^{\prime}}\left(z^{\prime}\right)$, on $G_{r^{\prime}}\left(z^{\prime}\right)$ we have $\partial \mathcal{B}_{z}^{\infty, *} \cap B_{r^{\prime}}\left(z^{\prime}\right)=\partial \mathcal{B}_{D_{h^{*}}(0, z)}\left(0 ; D_{h^{*}}\right) \cap B_{r^{\prime}}\left(z^{\prime}\right)$. Right: The red curve now represents $\partial \mathcal{B}_{D_{h^{*}}(0, z)}\left(0 ; D_{h}\right)$. By property $(b)$ of $G_{r}(z)$, on $G_{r}(z)$ we have $\partial \mathcal{B}_{D_{h^{*}}(0, z)}\left(0 ; D_{h}\right) \backslash B_{r}(z)=\partial \mathcal{B}_{D_{h^{*}}(0, z)}\left(0 ; D_{h^{*}}\right) \backslash B_{r}(z)$. The rest of the argument consists of showing the conditional law of $D_{h^{*}}(0, z)$ given $h$ is mutually absolutely continuous w.r.t. Lebesgue measure on its support.

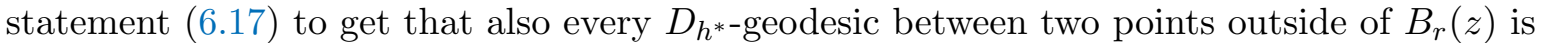
contained in $\mathbb{C} \backslash B_{r / A}(z)$. Since $h^{*}=h$ outside of $B_{r / A}(z)$, this implies that $\partial \mathcal{B}_{D_{h^{*}}(0, z)}\left(0 ; D_{h^{*}}\right) \backslash$ $B_{r}(z)=\partial \mathcal{B}_{D_{h^{*}}(0, z)}\left(0 ; D_{h}\right) \backslash B_{r}(z)$.

Thus, on the event $G_{r^{\prime}}\left(z^{\prime}\right) \cap G_{r}(z)$, a.s. $\partial \mathcal{B}_{D_{h^{*}}(0, z)}\left(0 ; D_{h}\right) \cap\left[B_{r^{\prime}}\left(z^{\prime}\right) \backslash B_{r}(z)\right]=\partial \mathcal{B}_{z}^{\infty, *} \cap\left[B_{r^{\prime}}\left(z^{\prime}\right) \backslash B_{r}(z)\right]$. Hence on this event a.s.

$$
\operatorname{dim}_{\mathcal{H}}^{0}\left(\partial \mathcal{B}_{D_{h^{*}}(0, z)}\left(0 ; D_{h}\right) \cap\left[B_{r^{\prime}}\left(z^{\prime}\right) \backslash B_{r}(z)\right]\right) \leq \Delta_{\text {ball }}^{0}
$$

For the rest of the proof we assume that the event $G_{r^{\prime}}\left(z^{\prime}\right) \cap G_{r}(z)$ occurs. Let

$$
\Lambda=\Lambda(h):=\left\{t \in \mathbb{R}: \operatorname{dim}_{\mathcal{H}}^{0}\left(\partial \mathcal{B}_{t}\left(0 ; D_{h}\right) \cap\left[B_{r^{\prime}}\left(z^{\prime}\right) \backslash B_{r}(z)\right]\right)>\Delta_{\text {ball }}^{0}\right\} .
$$

By (6.19), on the $\sigma(h)$-measurable event $G_{r^{\prime}}\left(z^{\prime}\right) \cap G_{r}(z)$, a.s.

$$
\mathbb{P}\left[D_{h^{*}}(0, z) \in \Lambda \mid h\right]=0 .
$$

To prove the lemma, we need to show that a.s. the Lebesgue measure of $\Lambda \cap\left(D_{h}\left(0, B_{r / A}(z)\right), \infty\right)$ is zero.

For $x \in \mathbb{R}$, let $f(x):=D_{h-(h, \phi)_{\nabla} \phi+x \phi}(0, z)$. By (6.20) and since we are assuming that $G_{r^{\prime}}\left(z^{\prime}\right) \cap$ $G_{r}(z)$ occurs, a.s.

$$
0=\mathbb{P}\left[D_{h^{*}}(0, z) \in \Lambda \mid h\right]=\mathbb{P}[f(Z) \in \Lambda \mid h]=P\left[Z \in f^{-1}(\Lambda) \mid h\right] .
$$

Since $Z$ is a standard Gaussian random variable independent from $h$, its conditional law given $h$ is mutually absolutely continuous w.r.t. Lebesgue measure on $\mathbb{R}$. So, a.s. the set $f^{-1}(\Lambda)$ has zero 
Lebesgue measure. Since $\phi$ is positive on $B_{r / A}(z)$ and zero outside $B_{r / A}(z)$, we deduce from the Weyl scaling property of the metric (Axiom III) that a.s. for every $x<y$,

$$
0 \leq f(y)-f(x) \leq\left(e^{\xi(y-x)\|\phi\|_{\infty}}-1\right) f(x) \leq\left(e^{\xi(y-x)\|\phi\|_{\infty}}-1\right) e^{\xi x\|\phi\|_{\infty}} D_{h-(h, \phi)_{\nabla} \phi}(0, z),
$$

where $\|\phi\|_{\infty}$ is the $L^{\infty}$ norm. We deduce that a.s. $f$ is locally Lipschitz, and so a.s. $f\left(f^{-1}(\Lambda)\right)$ has Lebesgue measure zero. Moreover, again by Axiom III, a.s. $f(\mathbb{R})=\left(D_{h}\left(0, B_{r / A}(z)\right), \infty\right)$, and therefore $f\left(f^{-1}(\Lambda)\right)=\Lambda \cap\left(D_{h}\left(0, B_{r / A}(z)\right), \infty\right)$. Thus, a.s. the Lebesgue measure of $\Lambda \cap$ $\left(D_{h}\left(0, B_{r / A}(z)\right), \infty\right)$ is zero, as desired.

We now apply Lemma 6.8 to prove the desired dimension result for $\partial \mathcal{B}_{t} \backslash \mathcal{Y}_{t}$.

Lemma 6.9. It holds almost surely that, for Lebesgue-a.e. $t>0$, the set $\partial \mathcal{B}_{t} \backslash \mathcal{Y}_{t}$ has Hausdorff dimension at most $\Delta_{\text {ball }}^{0}$.

Proof. Suppose that $w \in \partial \mathcal{B}_{t} \backslash \mathcal{Y}_{t}$ for some $t>0$. By the definition (6.2) of $\mathcal{Y}_{t}$, there exists a sequence of positive rational numbers $r_{n} \rightarrow 0$ and a sequence of points $z_{n} \in B_{r_{n}}(w) \cap \mathbb{Q}^{2}$ such that, for each $n$, the event $\left[F_{r_{n}}\left(z_{n}\right)\right]^{c}$ occurs. Recalling the definition (6.3) of $F_{\varepsilon}(z)$ (with $a$ and $b$ in (6.3) equal to $1 / A$ and $A^{2}$, respectively), we deduce that, for each $n$, the event $G_{r_{n}^{*}}\left(z_{n}\right)$ occurs for some $r_{n}^{*} \in\left[A r_{n}^{1 / 2}, 2 r_{n}^{1 / 4} / A^{2}\right] \cap \mathbb{Q}$. Observe that the balls $B_{r_{n}^{*}}\left(z_{n}\right)$ contain the point $w$ for all sufficiently large $n$ and that $r_{n} \rightarrow 0$ as $n \rightarrow \infty$. In other words, we have a sequence of balls $B_{r}(z)$ of arbitrarily small radii, all containing $w$, for which the corresponding events $G_{r}(z)$ occur. Since $t>0$ and $w \in \partial \mathcal{B}_{t} \backslash \mathcal{Y}_{t}$ were chosen arbitrarily, we deduce that, for each positive integer $k$, we can cover the set

$$
\bigcup_{t>0}\left(\partial \mathcal{B}_{t} \backslash \mathcal{Y}_{t}\right)
$$

by a collection $\mathfrak{B}_{k}$ of balls $B_{r}(z)$, with rational centers and rational radii which are at most $1 / k$, for which the corresponding events $G_{r}(z)$ occur. The union $\mathfrak{B}=\bigcup_{k} \mathfrak{B}_{k}$ of these collections is countable since the balls all have rational centers and radii. By Lemma 6.8, the following holds almost surely: for almost every $t>0$,

$$
\operatorname{dim}_{\mathcal{H}}\left(\partial \mathcal{B}_{t} \cap\left[B_{r^{\prime}}\left(z^{\prime}\right) \backslash B_{r}(z)\right]\right) \leq \Delta_{\text {ball }}^{0}
$$

for any nested pair of balls $B_{r}(z) \subset B_{r^{\prime}}\left(z^{\prime}\right)$ in $\mathfrak{B}$ for which $B_{r / A}(z) \subset B_{r^{\prime} / A}\left(z^{\prime}\right)$. Since every point in the set (6.22) is contained in a sequence of balls in $\mathfrak{B}$ of arbitrarily small radii, we deduce that a.s., it is the case that, for almost every $t$ and any point $w \in \partial \mathcal{B}_{t} \backslash \mathcal{Y}_{t}$, the intersection of $\partial \mathcal{B}_{t}$ with some element in $\mathfrak{B}$ containing $w$ has Hausdorff dimension at most $\Delta_{\text {ball }}^{0}$. By the countable stability of Hausdorff dimension, we conclude that a.s., the Hausdorff dimension of the entire set $\partial \mathcal{B}_{t} \backslash \mathcal{Y}_{t}$ is bounded from above by $\Delta_{\text {ball }}^{0}$ for almost every $t>0$.

We now combine Lemmas 6.7 and 6.9 to prove Proposition 6.6, implementing the strategy we outlined above.

Proof of Proposition 6.6. By Lemma 6.7, there exists $q>0$ such that for each $t>0$, the Hausdorff dimension of the set $\mathcal{Y}_{t}$ is almost surely bounded from above by $2-\xi Q+\xi^{2} / 2-q$. On the other hand, Lemma 6.9 asserts that it is a.s. the case that, for almost every $t>0$, the set $\partial \mathcal{B}_{t} \backslash \mathcal{Y}_{t}$ a.s. has Hausdorff dimension at most $\Delta_{\text {ball }}^{0}$. Therefore, for such a choice of $t$, we have a.s. $\operatorname{dim}_{\mathcal{H}}^{0} \partial \mathcal{B}_{t} \leq \max \left\{\Delta_{\text {ball }}^{0}, 2-\xi Q+\xi^{2} / 2-q\right\}$. By [Gwy20a, Theorem 1.1], if we sample $t$ uniformly at random from $[0,1]$, independently from $h$, then for every $\varepsilon>0$ the Hausdorff dimension of $\partial \mathcal{B}_{t}$ is at least $2-\xi Q+\xi^{2} / 2-\varepsilon$ with positive probability. Therefore, on an event with positive probability,

$$
2-\xi Q+\xi^{2} / 2-\varepsilon \leq \operatorname{dim}_{\mathcal{H}}^{0} \partial \mathcal{B}_{t} \leq \max \left\{\Delta_{\text {ball }}^{0}, 2-\xi Q+\xi^{2} / 2-q\right\} .
$$

Since $\varepsilon>0$ is arbitrary, this implies that $\Delta_{\text {ball }}^{0} \geq 2-\xi Q+\xi^{2} / 2$, as desired. 
We now complete the proof of Theorem 1.7 by proving the following lemma, which we alluded to at the beginning of this subsection.

Lemma 6.10. For each fixed $z \in \mathbb{C}$, a.s.

$$
\operatorname{dim}_{\mathcal{H}}^{0} \partial \mathcal{B}_{D_{h}(0, z)} \leq 2-\xi Q+\xi^{2} / 2
$$

Proof. To prove the lemma, we will show that for each $r, \varepsilon>0$,

$$
\mathbb{P}\left[\operatorname{dim}_{\mathcal{H}}^{0}\left(\partial \mathcal{B}_{D_{h}(0, z)} \backslash B_{r}(z)\right) \leq 2-\xi Q+\xi^{2} / 2\right] \geq 1-\varepsilon .
$$

The lemma then follows by sending $r, \varepsilon \rightarrow 0$.

By Lemma 4.8, we can choose $\widetilde{A}=\widetilde{A}(\varepsilon)>1$ such that, with probability $1-\varepsilon$, each $D_{h}$-geodesic between points of $\mathbb{C} \backslash B_{r}(z)$ is contained in $\mathbb{C} \backslash \overline{B_{r / \widetilde{A}}(z)}$ and more strongly there is a path $\pi$ in $B_{r}(z) \backslash \overline{B_{r / \widetilde{A}}(z)}$ such that

$$
\left(D_{h} \text {-length of } \pi\right)<D_{h}\left(\pi, B_{r / \widetilde{A}}(z)\right) .
$$

(We have introduced the tilde to avoid confusing this $\widetilde{A}$ with the constant $A$ we have been referencing throughout this subsection.) Let $E_{\varepsilon}$ be the event that this is the case. We henceforth work on the event $E_{\varepsilon}$.

As in the proof of Lemma 6.8, let $\phi: \mathbb{C} \rightarrow[0, \infty)$ be a smooth bump function which is positive on $B_{r / \widetilde{A}}(z)$, is zero outside $B_{r / \widetilde{A}}(z)$, and is normalized so that the Dirichlet energy $(\phi, \phi)_{\nabla}$ is 1 . Here, we also stipulate that $\phi$ is identically equal to some constant $c$ on the annulus $B_{r /(2 \widetilde{A})}(z) \backslash B_{r /(3 \widetilde{A})}(z)$. As before, we let $Z$ be a standard Gaussian random variable which is independent from $h$, and we consider the field

$$
h^{*}:=h-(h, \phi)_{\nabla} \phi+Z \phi
$$

By the definition of the whole-plane GFF, $h^{*}$ and $h$ are equal in distribution modulo additive constant.

Define

$$
\Lambda:=\left\{t>0: \operatorname{dim}_{\mathcal{H}}^{0}\left(\partial \mathcal{B}_{t}\left(0 ; D_{h}\right) \backslash B_{r}(z)\right)>2-\xi Q+\xi^{2} / 2\right\} .
$$

By [Gwy20a, Theorem 1.1], the set $\Lambda$ a.s. has Lebesgue measure zero.

For $x \in \mathbb{R}$, let $f(x):=D_{h-(h, \phi)_{\nabla} \phi+x \phi}(0, z)$, and note that $D_{h^{*}}(0, z)=f(Z)$. By [Gwy20a, Lemma 2.5] (with the field $h$ in that lemma replaced by $h-(h, \phi)_{\nabla} \phi$ and $\phi$ defined to equal $c$ on the annulus $\mathcal{A}$ in the lemma instead of 1$)$, it is a.s. the case that for every $x<y$,

$$
f(y)-f(x) \geq C_{h}(y-x) e^{\xi c x},
$$

where $C_{h}>0$ is measurable with respect to $\sigma(h)$. We deduce that a.s. (w.r.t. the law of $h$ ) the set $f^{-1}(\Lambda)$ has Lebesgue measure zero. Since the conditional law of $Z$ given $h$ is mutually absolutely continuous w.r.t. Lebesgue measure, $D_{h^{*}}(0, z) \notin \Lambda$ almost surely. In other words,

$$
\operatorname{dim}_{\mathcal{H}}^{0}\left(\partial \mathcal{B}_{D_{h^{*}}(0, z)}\left(0 ; D_{h}\right) \backslash B_{r}(z)\right) \leq 2-\xi Q+\xi^{2} / 2 \quad \text { a.s. }
$$

Since we are working on the event $E_{\varepsilon}$ and $\left.h\right|_{\mathbb{C} \backslash B_{r / \widetilde{A}}(z)}=\left.h^{*}\right|_{\mathbb{C} \backslash B_{r / \widetilde{A}}(z)}$, the same argument preceding (6.19) in the proof of Lemma 6.8 gives

$$
\partial \mathcal{B}_{D_{h^{*}}(0, z)}\left(0 ; D_{h}\right) \backslash B_{r}(z)=\partial \mathcal{B}_{D_{h^{*}}(0, z)}\left(0 ; D_{h^{*}}\right) \backslash B_{r}(z) .
$$

Hence (6.25) implies that

$$
\operatorname{dim}_{\mathcal{H}}^{0}\left(\partial \mathcal{B}_{D_{h^{*}}(0, z)}\left(0 ; D_{h^{*}}\right) \backslash B_{r}(z)\right) \leq 2-\xi Q+\xi^{2} / 2 \quad \text { a.s. on } E_{\varepsilon}
$$

Since $h^{*} \stackrel{d}{=} h$ and $\mathbb{P}\left[E_{\varepsilon}\right] \geq 1-\varepsilon$, this proves (6.23), and hence the lemma. 
Proof of Theorem 1.7. Combining the results of Proposition 6.5, Lemma 6.10, and Proposition 6.6 yields that for each fixed $z \in \mathbb{C}$, a.s.

$$
\Delta_{\text {ball }}^{0} \leq \operatorname{dim}_{\mathcal{H}}^{0} \partial \mathcal{B}_{D_{h}(0, z)} \leq 2-\xi Q+\xi^{2} / 2 \leq \Delta_{\text {ball }}^{0}
$$

\subsection{The exterior boundary of a metric ball}

We now study the exterior boundary of an LQG metric ball, which we defined in Definition 1.13. This random fractal satisfies a zero-one law analogous to the zero-one law for LQG metric ball boundaries that we stated in Theorem 6.5. Consider the infinite-volume "metric ball" $\mathcal{B}_{0}^{\infty}$ we defined in Proposition 4.7, and define its exterior boundary $\mathcal{O}_{0}^{\infty}$ in a manner analogous to Definition 1.13-i.e., as the union of the boundaries of the connected components of $\mathbb{C} \backslash \mathcal{B}_{0}^{\infty}$.

Proposition 6.11. There are deterministic constants $\Delta_{\text {out }}^{0}, \Delta_{\text {out }}^{\gamma}>0$ such that a.s. $\operatorname{dim}_{\mathcal{H}}^{0} \partial \mathcal{O}_{0}^{\infty}=$ $\Delta_{\text {out }}^{0}$ and $\operatorname{dim}_{\mathcal{H}}^{\gamma} \partial \mathcal{O}_{0}^{\infty}=\Delta_{\text {out }}^{\gamma}$. Furthermore, for each fixed $z \in \mathbb{C}$ a.s. $\operatorname{dim}_{\mathcal{H}}^{0} \mathcal{O}_{z}\left(w ; D_{h}\right) \geq \Delta_{\text {out }}^{0}$ and $\operatorname{dim}_{\mathcal{H}}^{\gamma} \mathcal{O}_{z}\left(w ; D_{h}\right) \geq \Delta_{\text {out }}^{\gamma}$ simultaneously for each $w \in \mathbb{C}$.

Proof. This follows from exactly the same argument used in the proof of Proposition 6.5.

The set $\mathbb{C} \backslash \overline{\mathcal{B}_{0}^{\infty}}$ has at most countably many connected components (since each component contains a point of $\mathbb{Q}^{2}$ ). By the countable stability of Hausdorff dimension $\operatorname{dim}_{\mathcal{H}}^{0} \mathcal{O}_{0}^{\infty}$ (resp. $\operatorname{dim}_{\mathcal{H}}^{\gamma} \mathcal{O}_{0}^{\infty}$ ) is a.s. equal to the supremum of the Euclidean (resp. $\gamma$-quantum) dimensions of the boundaries of the connected components of $\mathbb{C} \backslash \overline{\mathcal{B}_{0}^{\infty}}$. We expect that a.s. the boundary of each of these connected components have Euclidean dimension $\Delta_{\text {out }}^{0}$ and $\gamma$-quantum dimension $\Delta_{\text {out }}^{\gamma}$, but we do not prove this here.

As we described in Section 1, the points in $\partial \mathcal{B}_{s} \backslash \mathcal{O}_{s}$ are the points which are not on the boundary of any connected component of $\mathbb{C} \backslash \mathcal{B}_{s}$, which can arise as accumulation points of connected components of $\mathbb{C} \backslash \mathcal{B}_{s}$ with arbitrarily small diameters. The rest of this subsection is devoted to proving Theorem 1.14, which asserts that, at least with positive probability, the Euclidean and $\gamma$-quantum dimensions of $\mathcal{O}_{s}$ are strictly smaller than those of $\partial \mathcal{B}_{s}$. See Figure 9 for an illustration and outline of the proof of Theorem 1.14.

The proof is based on the generalized upper bound in Theorem 6.1. We will apply Theorem 6.1 for the events $F_{\varepsilon}(z)$ we constructed in Lemma 6.2 , with $a=1 / 2$ and $b=1$, and with the "good" events $G_{r}(z)$ defined as

$$
\begin{aligned}
G_{r}(z):= & \text { the event that there is a path in the annulus } B_{2 r}(z) \backslash B_{r}(z) \\
& \text { and whose } D_{h} \text {-length is shorter than } D_{h}\left(\partial B_{r / 2}(z), \partial B_{r}(z)\right)
\end{aligned}
$$

To apply Theorem 6.1 via Lemma 6.2 , we need to check that $G_{r}(z)$ satisfies the conditions of the lemma.

Lemma 6.12. The events $G_{r}(z)$ satisfy the conditions of Lemma 6.2.

Proof. First, by locality and Weyl scaling (Axioms II and III), $G_{r}(z)$ is a.s. determined by $\left.h\right|_{B_{2 r}(z) \backslash B_{r / 2}(z)}$ viewed modulo additive constant.

Second, by Axioms III and IV and the scale invariance of the law of $h$ modulo additive constant, $\mathbb{P}\left[G_{r}(z)\right]$ does not depend on $r$ or $z$. Moreover, it is easy to check that $\mathbb{P}\left[G_{r}(z)\right]>0$ for each fixed choice of $r$ and $z$ (see, e.g., [Gwy20a, Lemma 6.1]). 


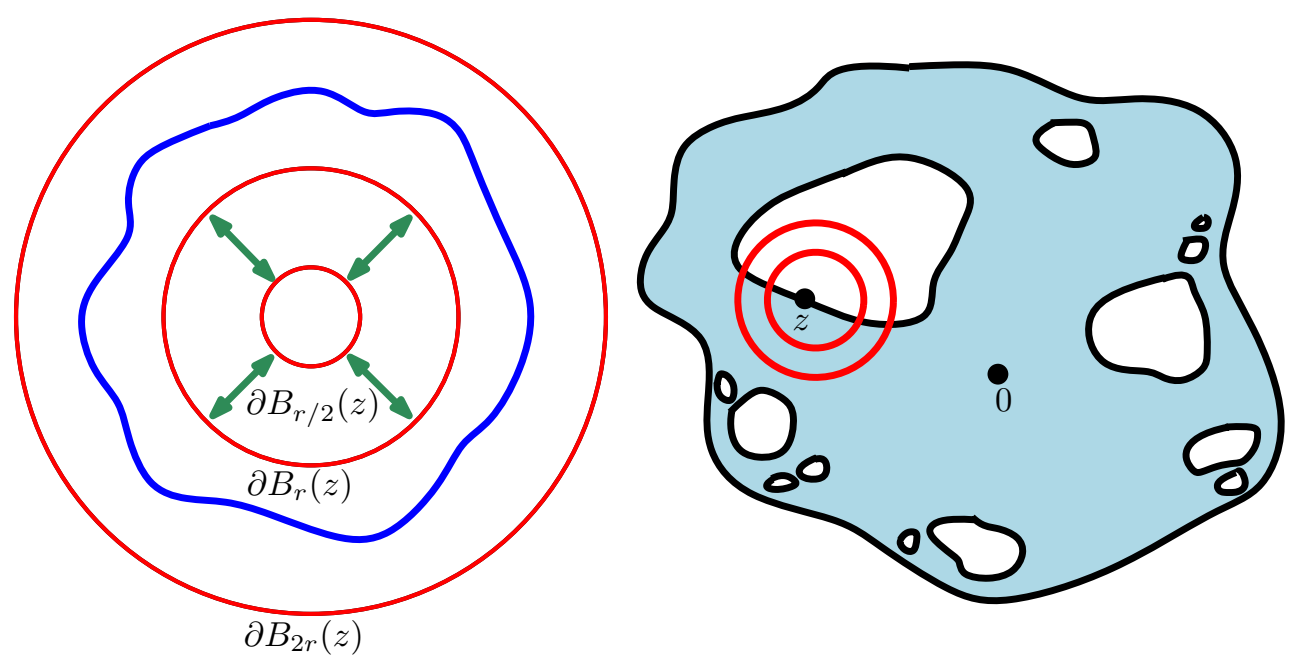

Figure 9: Illustration of the main ideas of the proof of Theorem 1.14. Left. We define $G_{r}(z)$ to be the event that there is a path in $B_{2 r}(z) \backslash B_{r}(z)$ which disconnects its inner and exterior boundaries (blue) whose $D_{h}$-length is less than $D_{h}\left(\partial B_{r}(z), \partial B_{r / 2}(z)\right)$. Then $G_{r}(z)$ satisfies the two conditions in Lemma 6.2 for a "good" event. This means that, if we define the event $F_{\varepsilon}(z)$ as in the statement of that lemma, $F_{\varepsilon}(z)$ satisfies the conditions of the generalized upper bound (Theorem 6.1). Right. Theorem 6.1 allows us to reduce our task of proving Theorem 1.14 to showing that, if $z \in \mathcal{O}_{s}$, then the event $F_{\varepsilon}(w)$ occurs for every small enough $\varepsilon>0$ and every $w \in B_{\varepsilon}(z) \cap \mathbb{Q}^{2}$. This is the case because a point on the exterior boundary $\mathcal{O}_{s}$ has the following property: for every sufficiently small Euclidean annulus $A$ whose inner disk contains $z$, the $D_{h}$-distance from the inner boundary of $A$ to $z$ must be shorter than the minimal $D_{h}$-length of the paths in $A$ which disconnect its inner and exterior boundaries. Otherwise, $z$ would not be on the exterior boundary, since the metric ball growth $\left\{\mathcal{B}_{s}\right\}_{s \geq 0}$ would form arbitrarily small "bubbles" containing $z$ before reaching $z$.

Proof of Theorem 1.14. We will prove the dimension upper bounds for the exterior boundary of a metric balls of a fixed radius. One can then use exactly the same argument we used in our proof of Lemma 6.10 to deduce the result for the exterior boundary of a metric ball stopped when it hits a fixed point.

By combining Lemmas 6.12 and 6.2, we deduce that the hypotheses of Theorem 6.1 are satisfied for the events $F_{\varepsilon}(z)$ of (6.3) with the above choice of $G_{r}(z)$. Define $\mathcal{Y}_{s}$ for $s>0$ as in Theorem 6.1 for the above choice of $F_{\varepsilon}(z)$. Then $\mathcal{Y}_{s}$ satisfies the dimension upper bounds of Theorem 6.1. These are exactly the bounds we want to prove for $\mathcal{O}_{s}$. We will prove these bounds for $\mathcal{O}_{s}$ by showing that $\mathcal{O}_{s} \subset \mathcal{Y}_{s}$.

Suppose $z \in \mathcal{O}_{s}$. Let $\varepsilon>0$ be small enough that $0 \notin B_{100 \varepsilon^{1 / 4}}(z)$ and $\partial B_{100 \varepsilon^{1 / 4}}(z)$ intersects the connected component of $\partial \mathcal{B}_{s}$ which contains $z$. Let $w \in B_{\varepsilon}(z) \cap \mathbb{Q}^{2}$. We claim that $F_{\varepsilon}(w)$ occurs.

Indeed, if $F_{\varepsilon}(w)$ does not occur then by definition there is some $r \in\left[\varepsilon^{1 / 2}, \varepsilon^{1 / 4}\right] \cap \mathbb{Q}$ for which $G_{r}(w)$ occurs, i.e., there is a path $\pi$ in $B_{2 r}(w) \backslash B_{r}(w)$ which disconnects the inner and exterior boundaries of $B_{2 r}(w) \backslash B_{r}(w)$ and whose $D_{h}$-length is shorter than $D_{h}\left(\partial B_{r / 2}(w), \partial B_{r}(w)\right)$. Let $P$ be a $D_{h}$-geodesic from 0 to $z$. Since $z \in B_{r / 2}(w), P$ must hit the path $\pi$ and then subsequently cross from $\partial B_{r}(w)$ to $\partial B_{r / 2}(w)$. Since $P$ is a $D_{h}$-geodesic and the $D_{h}$-length of $\pi$ is shorter than $D_{h}\left(\partial B_{r / 2}(w), \partial B_{r}(w)\right)$, this implies that $\pi \subset \mathcal{B}_{s}$. But, $\pi$ disconnects $z$ from $\partial B_{100 \varepsilon^{1 / 4}}(z)$. This is a contradiction since we have assumed that $\varepsilon$ is small enough so that $\partial B_{100 \varepsilon^{1 / 4}}(z)$ intersects the connected component of $\partial \mathcal{B}_{s}$ which contains $z$. Therefore $F_{\varepsilon}(w)$ occurs, and so $\mathcal{O}_{s} \subset \mathcal{Y}_{s}$.

Finally, to get that with positive probability $\partial \mathcal{B}_{s} \backslash \mathcal{O}_{s}$ is uncountable, we observe that [Gwy20a, 
Theorem 1.1] shows that, with positive probability, $\operatorname{dim}_{\mathcal{H}}^{0} \partial \mathcal{B}_{s}>2-\xi Q+\xi^{2} / 2-q \geq \operatorname{dim}_{\mathcal{H}}^{0} \mathcal{O}_{s}$. If this is the case then there must be uncountably many points in $\partial \mathcal{B}_{s} \backslash \mathcal{O}_{s}$. Similarly, since Theorem 1.7 shows that, almost surely, $\operatorname{dim}_{\mathcal{H}}^{0} \partial \mathcal{B}_{D_{h}(0, z)}>2-\xi Q+\xi^{2} / 2-q \geq \operatorname{dim}_{\mathcal{H}}^{0} \mathcal{O}_{D_{h}(0, z)}$, we deduce that $\partial \mathcal{B}_{s} \backslash \mathcal{O}_{s}$ a.s. contains uncountably many points.

\section{References}

[AKM17] O. Angel, B. Kolesnik, and G. Miermont. Stability of geodesics in the Brownian map. Ann. Probab., 45(5):3451-3479, 2017, 1502.04576. MR3706747

[Ang19] M. Ang. Comparison of discrete and continuum Liouville first passage percolation. Electron. Commun. Probab., 24:Paper No. 64, 12, 2019, 1904.09285. MR4029433

[BBI01] D. Burago, Y. Burago, and S. Ivanov. A course in metric geometry, volume 33 of Graduate Studies in Mathematics. American Mathematical Society, Providence, RI, 2001. MR1835418

[Bef08] V. Beffara. The dimension of the SLE curves. Ann. Probab., 36(4):1421-1452, 2008, math/0211322. MR2435854 (2009e:60026)

[Ber] N. Berestycki. Introduction to the Gaussian Free Field and Liouville Quantum Gravity. Available at https://homepage.univie.ac.at/nathanael.berestycki/articles. html.

[BM17] J. Bettinelli and G. Miermont. Compact Brownian surfaces I: Brownian disks. Probab. Theory Related Fields, 167(3-4):555-614, 2017, 1507.08776. MR3627425

[Dav88] F. David. Conformal field theories coupled to 2-D gravity in the conformal gauge. Mod. Phys. Lett. A, 3(17), 1988.

[DDDF20] J. Ding, J. Dubédat, A. Dunlap, and H. Falconet. Tightness of Liouville first passage percolation for $\gamma \in(0,2)$. Publ. Math. Inst. Hautes Études Sci., 132:353-403, 2020, 1904.08021. MR4179836

$\left[\mathrm{DFG}^{+} 20\right]$ J. Dubédat, H. Falconet, E. Gwynne, J. Pfeffer, and X. Sun. Weak LQG metrics and Liouville first passage percolation. Probab. Theory Related Fields, 178(1-2):369-436, 2020, 1905.00380. MR4146541

[DG18] J. Ding and E. Gwynne. The fractal dimension of Liouville quantum gravity: universality, monotonicity, and bounds. Communications in Mathematical Physics, 374:1877-1934, 2018, 1807.01072.

[DG19] J. Ding and S. Goswami. Upper bounds on Liouville first-passage percolation and Watabiki's prediction. Comm. Pure Appl. Math., 72(11):2331-2384, 2019, 1610.09998. MR4011862

[DK89] J. Distler and H. Kawai. Conformal field theory and 2D quantum gravity. Nucl.Phys. B, 321(2), 1989.

[DS11] B. Duplantier and S. Sheffield. Liouville quantum gravity and KPZ. Invent. Math., 185(2):333-393, 2011, 1206.0212. MR2819163 (2012f:81251) 
[DZZ19] J. Ding, O. Zeitouni, and F. Zhang. Heat kernel for Liouville Brownian motion and Liouville graph distance. Comm. Math. Phys., 371(2):561-618, 2019, 1807.00422. MR4019914

[GM20a] E. Gwynne and J. Miller. Confluence of geodesics in Liouville quantum gravity for $\gamma \in(0,2)$. Ann. Probab., 48(4):1861-1901, 2020, 1905.00381. MR4124527

[GM20b] E. Gwynne and J. Miller. Local metrics of the Gaussian free field. Ann. Inst. Fourier (Grenoble), 70(5):2049-2075, 2020, 1905.00379. MR4245606

[GM21a] E. Gwynne and J. Miller. Conformal covariance of the Liouville quantum gravity metric for $\gamma \in(0,2)$. Ann. Inst. Henri Poincaré Probab. Stat., 57(2):-, 2021, 1905.00384. MR4260493

[GM21b] E. Gwynne and J. Miller. Existence and uniqueness of the Liouville quantum gravity metric for $\gamma \in(0,2)$. Invent. Math., 223(1):213-333, 2021, 1905.00383. MR4199443

[GMS19] E. Gwynne, J. Miller, and S. Sheffield. Harmonic functions on mated-CRT maps. Electron. J. Probab., 24:no. 58, 55, 2019, 1807.07511.

[GP19a] E. Gwynne and J. Pfeffer. Bounds for distances and geodesic dimension in Liouville first passage percolation. Electronic Communications in Probability, 24:no. 56, 12, 2019, 1903.09561.

[GP19b] E. Gwynne and J. Pfeffer. KPZ formulas for the Liouville quantum gravity metric. Transactions of the American Mathematical Society, to appear, 2019.

[Gwy20a] E. Gwynne. The Dimension of the Boundary of a Liouville Quantum Gravity Metric Ball. Comm. Math. Phys., 378(1):625-689, 2020, 1909.08588. MR4124998

[Gwy20b] E. Gwynne. Geodesic networks in Liouville quantum gravity surfaces. Probability and Mathematical Physics, to appear, 2020, 2010.11260.

[Gwy20c] E. Gwynne. Random surfaces and Liouville quantum gravity. Notices Amer. Math. Soc., 67(4):484-491, 2020, 1908.05573. MR4186266

[HMP10] X. Hu, J. Miller, and Y. Peres. Thick points of the Gaussian free field. Ann. Probab., 38(2):896-926, 2010, 0902.3842. MR2642894 (2011c:60117)

[HS18] N. Holden and X. Sun. SLE as a mating of trees in Euclidean geometry. Comm. Math. Phys., 364(1):171-201, 2018, 1610.05272. MR3861296

[Kah85] J.-P. Kahane. Sur le chaos multiplicatif. Ann. Sci. Math. Québec, 9(2):105-150, 1985. MR829798 (88h:60099a)

[KPZ88] V. Knizhnik, A. Polyakov, and A. Zamolodchikov. Fractal structure of 2D-quantum gravity. Modern Phys. Lett A, 3(8):819-826, 1988.

[Le 10] J.-F. Le Gall. Geodesics in large planar maps and in the Brownian map. Acta Math., 205(2):287-360, 2010, 0804.3012. MR2746349 (2012b:60272)

[Le 13] J.-F. Le Gall. Uniqueness and universality of the Brownian map. Ann. Probab., 41(4):28802960, 2013, 1105.4842. MR3112934 
[Mie13] G. Miermont. The Brownian map is the scaling limit of uniform random plane quadrangulations. Acta Math., 210(2):319-401, 2013, 1104.1606. MR3070569

[MP10] P. Mörters and Y. Peres. Brownian motion. Cambridge Series in Statistical and Probabilistic Mathematics. Cambridge University Press, Cambridge, 2010. With an appendix by Oded Schramm and Wendelin Werner. MR2604525 (2011i:60152)

[MQ20a] J. Miller and W. Qian. Geodesics in the Brownian map: Strong confluence and geometric structure. ArXiv e-prints, August 2020, 2008.02242.

[MQ20b] J. Miller and W. Qian. The geodesics in Liouville quantum gravity are not SchrammLoewner evolutions. Probab. Theory Related Fields, 177(3-4):677-709, 2020, 1812.03913.

[MS17] J. Miller and S. Sheffield. Imaginary geometry IV: interior rays, whole-plane reversibility, and space-filling trees. Probab. Theory Related Fields, 169(3-4):729-869, 2017, 1302.4738. MR3719057

[MS20] J. Miller and S. Sheffield. Liouville quantum gravity and the Brownian map I: the $\mathrm{Q} L E(8 / 3,0)$ metric. Invent. Math., 219(1):75-152, 2020, 1507.00719. MR4050102

[MS21a] J. Miller and S. Sheffield. An axiomatic characterization of the Brownian map. J. Éc. polytech. Math., 8:609-731, 2021, 1506.03806. MR4225028

[MS21b] J. Miller and S. Sheffield. Liouville quantum gravity and the Brownian map II: Geodesics and continuity of the embedding. Ann. Probab., 49(6):2732-2829, 2021, 1605.03563. MR4348679

[MS21c] J. Miller and S. Sheffield. Liouville quantum gravity and the Brownian map III: the conformal structure is determined. Probab. Theory Related Fields, 179(3-4):1183-1211, 2021, 1608.05391. MR4242633

[MWW16] J. Miller, S. S. Watson, and D. B. Wilson. Extreme nesting in the conformal loop ensemble. Ann. Probab., 44(2):1013-1052, 2016, 1401.0218. MR3474466

[Pol81] A. M. Polyakov. Quantum geometry of bosonic strings. Phys. Lett. B, 103(3):207-210, 1981. MR623209 (84h:81093a)

[Pom92] C. Pommerenke. Boundary behaviour of conformal maps, volume 299 of Grundlehren der Mathematischen Wissenschaften [Fundamental Principles of Mathematical Sciences]. Springer-Verlag, Berlin, 1992. MR1217706 (95b:30008)

[RV11] R. Rhodes and V. Vargas. KPZ formula for log-infinitely divisible multifractal random measures. ESAIM Probab. Stat., 15:358-371, 2011, 0807.1036. MR2870520

[She07] S. Sheffield. Gaussian free fields for mathematicians. Probab. Theory Related Fields, 139(3-4):521-541, 2007, math/0312099. MR2322706 (2008d:60120)

[Wat93] Y. Watabiki. Analytic study of fractal structure of quantized surface in two-dimensional quantum gravity. Progr. Theor. Phys. Suppl., (114):1-17, 1993. Quantum gravity (Kyoto, 1992).

[WP20] W. Werner and E. Powell. Lecture notes on the Gaussian Free Field. ArXiv e-prints, April 2020, 2004.04720. 